\title{
Identification and characterization of virulence associated factors of $C$. jejuni
}

\author{
Dissertation \\ Submitted for the acquisition of Doctorate degree in Biology \\ Faculty of Biological Sciences \\ School of Natural Sciences and Mathematics \\ Georg August University \\ Göttingen, Germany
}

Submitted by

Abdul Malik

from

Quetta-Pakistan

GÖTTINGEN, 2010 
The experimental part of this PhD thesis was done under the supervision of

Prof. Dr. Uwe Groß and Dr. Raimond Lugert in the

Institute of Hygiene and Medical Microbiology

Georg-August-University of Göttingen

Göttingen - Germany.

2010 
To my family, especially my wife

To my teachers 
With the exception of references to the work of other scientists, I hereby declare that this thesis entitled "Identification and characterization of virulence associated factors of $C$. jejuni" all the work described in this thesis is my own work and that, to the best of my knowledge and belief, it contains no material previously published or written by another person nor submitted, neither in whole or in part, elsewhere to any other institution for degree, diploma other qualification. 


\section{List of Publications}

Tareen A.M., Dasti J.I., Zautner A.E., Groß U. and Lugert R. (2010). Campylobacter jejuni proteins Cj0952c and Cj0951c affect the chemotactical behavior towords formic acid and are important for the invasion of the host cells. Microbiology. [Epub ahead of print]

Dasti J.I.,Tareen A.M., Lugert R., Zautner A.E. and Groß U. (2009). Campylobacter jejuni: a brief overview on pathogenicity-associated factors and disease-mediating mechanisms. Int J Med Microbiol. 300: 205-211.

Tareen A.M., Dasti J.I., Zautner A.E., Groß U. and Lugert R. (2010). Sulphite: cytochrome c oxidoreductase deficiency in Campylobacter jejuni strongly reduced the infectivity of the pathogen. Microbiology (submitted). 


\section{ACKNOWLEDGMENT}

In the name of Allah, the Most Gracious and the Most Merciful I want to express my deeply-felt thanks to Allah for the strengths and His blessing in completing this thesis.

After word, I thank my supervisor Prof. Dr. med. Uwe Groß. It has been an honor to be his Ph.D. student. He was always there to listen and to give advice. I appreciate all his contributions of time, wisdom, knowledge and funding to make my Ph.D. experience productive and stimulating. He taught me how to ask questions and express my ideas. Thank you Prof. Groß for giving me the opportunity to attend a variety of conferences and being the first referee of my thesis

I would like to express my particular gratitude to Dr. Raimond Lugert, my working supervisor. He showed me different ways to come up a research problem and the need to be persistent to accomplish any goal. His insights have strengthened this study significantly. His broad discussions around my work and interesting explorations in operations have been very helpful for this study. Throughout my thesis writing period, he provided encouragement, good teaching, sound advice, good company, and lots of good ideas. I would have been lost without him.

My warm thanks are due to PD Dr. Kramer, who is the second referee of this thesis. Many thanks to Prof. Dr. Stülke, PD Dr. Daniel, Prof. Dr. Wienands and Prof. Dr. Pöggeler for being my examination committee members.

I warmly thank Dr. Javid lqbal Dasti for his initial guidance and help. In addition, I like to thank Dr. Andreas Zautner for his company and suggestions during my research.

I also acknowledge my colleagues Steffen, Sara, Jasmin, Anna, Kristen, Friedrich, Diana, Albert, Karen, Ayu, Marrot, Sherani, Adnan, Sajad, Hazir and technical 
persons Mrs. C. Kunze, Mrs. S. Rabenda, Mrs. U. Bölling, Mrs A. Siems and Mrs. R. Althaus from institute and friends Saleeh, Muhammad Haroon, Abid and especially the whole Pakistani community living in Germany with whom I had a pleasant, enjoyable and fruitful company. I am also very great full to Prof. C. Lüder, Dr. W. Bohne, Dr. Oliver Bader and Prof. Dr. med. H. Eiffert for their technical support and all the member of the institute of Medical Microbiology for providing me a very friendly and nice working environment

Great thanks to my wife and our children Sana and Abdul Rafeh for their love, patience and encouragement that have upheld me, especially in those days in where I spent more time in the lab than at home.

My specially thanks to Deutscher Akademischer Austauschdienst (DAAD), Higher education commission Pakistan and University of Balochistan for the financial support which gave me the chance to pursuit my PhD study in Germany.

Last but not the least; I would like to acknowledge the role of my parents and other family members especially my best friend Ali Anan Qamer in my success. Without them I would have never made it so far. 


\section{List of Contents}

List of Figure and Table I

List of Abbreviations III

Summary VI

1 Introduction 1

1.1 Campylobacter jejuni 1

1.1.1 C. jejuni caused diseases 3

1.2 General features of virulance associated factors of 4 C. jejuni

1.2.1 Flagellum 4

$\begin{array}{lll}1.2 .2 & \text { Invasion }\end{array}$

1.2.3 Chemotaxis 8

1.2.4 Cytolethal distending toxin 9

$\begin{array}{lll}1.2 .5 & \text { Translocation } & 10\end{array}$

1.3 Response to stress 11

1.4 Objective of the study 14

2 Materials and Methods $\quad 15$

2.1 Materials 15

$\begin{array}{lll}2.1 .1 & \text { Instruments } & 15\end{array}$

2.1.2 Cell culture media and additives 16

2.1.2.1 Antibiotics (disc) 16

2.1.2.2 Antibiotics (powder) 16

2.1.2.3 Media and additives 16

2.1.2.4 Disposable materials and plastic ware 17

$\begin{array}{lll}2.1 .2 .5 & \text { Cell lines } & 17\end{array}$

2.1.2.6 Bacterial strains and mutants 17

2.1.2.7 Bacterial culture media 17

2.1.3 Chemicals and reagents 18

2.1.3.1 Antibodies 18 
2.1.3.2 Membranes and filters 18

2.1.3.3 Enzymes 18

2.1.3.4 Standard Buffers 19

2.1.3.5 Kits and reagents 21

2.1.3.6 Oligonucletides 23

2.2 Methods 27

2.2.1 Cell culture 27

$\begin{array}{lll}\text { 2.2.1.1 Bacterial strains and culture conditions } & 27\end{array}$

2.2.1.2 Preparation of chemical competent E.coli cells 27

2.2.1.3 Generation of electrocompetent bacterial cells 28

2.2.1.4 Electroporation 28

2.2.1.5 Cultivation of eukaryotic cells 29

2.2.1.6 Invasion and adhesion assays $\quad 29$

2.2.1.7 Freezing and thawing of Caco2 cells 30

2.2.2 Modification of nucleic acids 30

2.2.2.1 Preparation of genomic DNA 30

2.2.2.2 Isolation of plasmid DNA 30

2.2.2.3 Isolation of RNA 31

2.2.2.4 Quantification of DNA and RNA 31

2.2.2.5 Polymerase chain reaction (PCR) 32

2.2.2.6 Conventional reverse transcriptase-PCR 32

2.2.2.7 Real-Time reverse transcriptase-PCR analysis 32

2.2.2.8 Enzymatic digestion of DNA 33

2.2.2.9 Purification of PCR products 33

2.2.2.10 Ligation 34

2.2.2.11 Direct sequencing of genomic DNA from C. jejuni 34

2.2.3 Experiments related to mutants of $C$. jejuni and their characterization $\quad 34$

2.2.3.1 Knock-out inactivation of cj0005c 34

2.2.3.2 Cloning of $C$. jejuni genes into expression vector pRRC 35

2.2.3.3 Motility assay 35

2.2.3.4 Chemotaxis assay $\quad 35$ 
2.2.3.5 Autoagglutination assay

2.2.4 Analysis of protein expression 36

2.2.4.1 Measurement of protein concentration 36

2.2.4.2 Sodium dodecy sulphate polyarcylamide gel electrophoresis 36 (SDS-PAGE)

2.2.4.3 Protein analysis by SDS-PAGE 36

2.2.4.4 Purification of $6 x$ His-tagged proteins from C. jejuni under 37 denaturing conditions

2.2.4.5 Western blotting 37

2.2.4.6 Statistical analysis 38

3 Results 39

3.1 Invasion capacity of $C$. jejuni isolate B2 and the $C$. jejuni strains 39 NCTC 11168 and 81-176

3.2 Identification of invasion related C. jejuni genes 40

3.3 Characterization of cj0952c 42

3.3.1 Transposone insertion in gene cj0952c does not alter the 42 transcription of both upstream gene cj0953c as well as downstream gene cj0951c

3.3.2 Functional restoration of $C$. jejuni B2 $\Delta c j 0952 c$

3.3.3 C. jejuni genes $c j 0952 c$ and $c j 0951 c$ of the clinical isolate B2 are 46 translated into separate proteins

3.3.4 Decreased motility of $C$. jejuni B2 $\Delta c j 0952 c \quad 48$

3.3.5 The C. jejuni protein Cj0952c and Cj0951c alters chemotactical 50 behavior of the pathogen in the presence of formic acid

3.3.6 The presence or absence of Cj0952c-Cj0951c does not 53 affect the usage of formic acid

3.4 Characterization of $c j 0005 c \quad 54$

3.4.1 Construction of a cj0005c knock-out mutant and complementation of 55 the mutant phenotype

3.4.2 The loss of a functional SOR leads to reduced growth in the 58 presence of sodium sulphite 
3.4.3 The absence of a functional SOR reduces the motility of $C$. jejuni 59

3.4.4 Reduced motility conduces to decreased invasiveness 59

3.4.5 Diminished adherence contribute to reduced invasiveness 60

3.4.6 The transcription of virulence associated genes is down-regulated 61 in the absence of a functional SOR

3.4.7 Reduced autoagglutianation of mutant B2::cj0005c 64

4 Discussion 66

5 References 74

6 Curriculum vitae 92 


\section{List of Figure and Tables}

Figure 1.1: Electron microscopy image of $C$. jejuni cells 1

Figure 1.2: The diagram of $C$. jejuni ecological cycle 2

Figure 1.3: A cross-sectional appearance of the C. jejuni cell wall 5

Figure 1.4: $\quad$ Pathogenesis model of $C$. jejuni $\quad 7$

Figure 3.1: $\quad$ Comparative analysis of the C. jejuni strains B2, 39 NCTC 11168 and 81-176

Figure 3.2: Invasion of the B2 wild-type strain and selected clones of a transposon based mutant library

Figure 3.3: Genomic composition of cj0953c, cj0952c, cj0951c and cj0950c in the clinical $C$. jejuni isolate B2

Figure 3.4: $\quad$ RT-PCR assays for the investigation of $C$. jejuni genes cj0951c, cj0952c and cj0953c in wild-type B2 strain and the mutant strain B2 $\Delta$ cj0952c

Figure 3.5: Infection of Caco2 cells by wild-type strain B2, the mutant and the complemented mutants

Figure 3.6: Detection of the recombinant protein in $C$. jejuni by immunobloting

Figure 3.7: Motility of wild type strain, complemented mutants and cj0952c-mutant of $C$. jejuni strain B2

Figure 3.8: Motility zones of the wild-type strain B2, the mutant B2 $\Delta c j 0952 c$ and the complemented mutants

Figure 3.9: Photographical representation of chemotactical behavior of the investigated strains in the presence of formic acid

Figure 3.10: Growth curve with and without $10 \mathrm{mM}$ formic acid

Figure 3.11: Recovery of $C$. jejuni bacteria after invasion assays on Caco2 cells

Figure 3.12: Genome arrangements, verification of cloning procedures and invasion assays of the strains under investigation

Figure 3.13: Growth curve in the presence and absence of $10 \mathrm{mM}$ $\mathrm{Na}_{2} \mathrm{SO}_{3}$ 
Figure 3.14: Motility assays and gentamycine protection assays after centrifugation

Figure 3.15: Adhesion assays 61

Figure 3.16: Autoagglutination 65

Table 2.1: HPLC-purified oligonucleotide primers used for 23 sequencing

Table 2.2: HPLC-purified oligonucleotide primers used for cloning in $\mathrm{pRRC}$ and pBluescript II

Table 2.3: Oligonucleotide primers used for semiquantitative real-time RT-PCR analysis

Table 3.1: $\quad$ Specification of the detected genes with regard to function and localization as far as known

Table 3.2: The mean values of colonies recovered after five independent invasion experiments

Table 3.3: Chemoattraction zones of $C$. jejuni wild type strain, complemented mutants and mutant are indicated in $\mathrm{mm}$

Table 3.4: Transcription level of selected virulence-associated genes 63 from C. jejuni in wild-type strain B2 and mutant B2::cj0005c

Table 3.5: Restored transcription levels of genes in the complemented 64 mutant compared to parental strain B2 


\section{List of Abbreviations}

\begin{tabular}{|c|c|}
\hline AMAN & Acute motor axonal neuropathy \\
\hline AMSAN & Acute motor axonal and sensory neuropathy \\
\hline ATP & Adenosine tri-phosphate \\
\hline BCA & Bicinchoninic acid \\
\hline BCIP & 5-bromo-4-chloro-3-indolylphosphate \\
\hline BLAST & Basic Local Alignment Search Tool \\
\hline bp & Base pairs \\
\hline BSA & Bovine serum albumin \\
\hline${ }^{0} \mathrm{C}$ & Centigrade \\
\hline Caco2 & Human colon carcinogenic \\
\hline $\mathrm{CaCl}_{2}$ & Calcium chloride \\
\hline $\mathrm{Cam}^{r}$ & Chloramphenicol resistance \\
\hline cDNA & Complementary DNA \\
\hline CDT & Cytolethal distending toxin \\
\hline $\mathrm{CHO}$ & Chinese hamster ovary \\
\hline cfu & Colony forming unit \\
\hline $\mathrm{cm}$ & Centimeter \\
\hline $\mathrm{Cp}$ & Crossing point \\
\hline $\mathrm{ddH}_{2} \mathrm{O}$ & Double distilled water \\
\hline DMEM & Dulbecco minimal essential medium \\
\hline DMSO & Dimethyl sulfoxide \\
\hline DNA & Deoxyribonucleic acid \\
\hline dNTP & Deoxyrinucleotide triphosphate \\
\hline ECL & Enhanced chemiluminescent \\
\hline EDTA & Ethylenediaminetetraacetic acid \\
\hline FCS & Fetal calf serum \\
\hline Fig. & Figure \\
\hline g & Force of gravity \\
\hline GBS & Guillain-Barre syndrome \\
\hline
\end{tabular}




\begin{tabular}{|c|c|}
\hline gDNA & Genomic DNA \\
\hline GIT & Gastrointestinal tract \\
\hline $\mathrm{HCl}$ & Hydrogen chloride \\
\hline HIV & Human immunodeficiency virus \\
\hline HPLC & High Performance Liquid Chromatography \\
\hline Hrs & Hours \\
\hline $\operatorname{kan}^{\mathrm{R}}$ & Kanamycin resistance \\
\hline $\mathrm{kb}$ & Kilo base pair \\
\hline $\mathrm{KCl}$ & Potassium chloride \\
\hline kDa & Kilo Dalton \\
\hline kV & Kilo volt \\
\hline LB & Luria broth \\
\hline M & Molar \\
\hline Mbp & Mega base pair \\
\hline MCPs & Methyl-accepting chemotaxis proteins \\
\hline $\mathrm{MH}$ & Mueller Hinton \\
\hline $\min$ & Minute \\
\hline $\mathrm{ml}$ & Milliliter \\
\hline $\mathrm{mm}$ & Millimeter \\
\hline $\mathrm{mM}$ & Millimole \\
\hline $\mathrm{MgCl}_{2}$ & Magnesium chloride \\
\hline $\mathrm{MnCl}_{2}$ & Manganese chloride \\
\hline $\mathrm{MOI}$ & Multiplicity of infection \\
\hline$\mu \mathrm{g}$ & Micrograme \\
\hline$\mu l$ & Microliter \\
\hline mRNA & Messenger ribonucleic acid \\
\hline NAD & Nicotineamide dinucleotide \\
\hline $\mathrm{NaCl}_{2}$ & Sodium chloride \\
\hline NADPH & $\begin{array}{l}\text { Reduced nicotinamide adenine dinucletide and } \\
\text { phosphate }\end{array}$ \\
\hline NBT & Nitroblue tetrazolium chloride \\
\hline
\end{tabular}




\begin{tabular}{|c|c|}
\hline NEAA & Non essential amino acid \\
\hline ND & NanoDrop \\
\hline Ni-NTA & Nickel-nitrilotriacetic acid \\
\hline $\mathrm{ng}$ & Nanogram \\
\hline OD & Optical density \\
\hline PAGE & Polyacrylamide gelelectrophoresis \\
\hline PBS & Phosphate buffered saline \\
\hline pmol & Pico mole \\
\hline PCR & Polymerase chain reaction \\
\hline $\mathrm{RbCl}_{2}$ & Rubidium Chloride \\
\hline RNA & Ribonucleic acid \\
\hline rpm & Round per minute \\
\hline RT & Room temperature \\
\hline RT-PCR & Reverse transcriptase-Polymerase chain reaction \\
\hline s & Seconds \\
\hline SDS & Sodiumdodecylsulfate \\
\hline SOR & Sulphite:cytochrome c oxidoreductase \\
\hline spp. & Species \\
\hline TEMED & N,N,N,N-Tetramethyl-Ethylenediamine \\
\hline TFB & Transformation buffer \\
\hline Tlp & Transducer-like protein \\
\hline Trp & Tryptophan \\
\hline$U$ & Unit \\
\hline UV & Ultraviolet \\
\hline $\mathrm{v} / \mathrm{v}$ & Volume/volume \\
\hline$w / v$ & Weight/volume \\
\hline
\end{tabular}




\section{SUMMARY}

Campylobacter jejuni, an important food-borne human bacterial pathogen in industrialized countries and in the developing world, the leading cause of bacterial diarrhoea. The infection may be accompanied by fever and abdominal cramps and, as a post-infection complication, in rare cases Guillain-Barré syndrome might emerge. In contrast, $C$. jejuni is only a commensal in poultry, where the pathogen predominantly resides in the cecum which contains many anaerobic fermentative bacteria. Within these natural habitats, $C$. jejuni is able to metabolize products like free amino and keto acids that have been generated by these bacterial species or from the host himself.

In order to identify genes which are related to the invasion of host cells by the pathogen, 660 clones of a transposon mutant library generated in the clinical $C$. jejuni isolate B2 were screened. Thereby, a clone with a transposon insertion in gene cj0952c was identified. As in the sequenced C. jejuni strain NCTC 11168, the respective protein together with the gene product of adjacent gene $c j 0951 \mathrm{c}$ consists of two transmembrane domains, a HAMP domain followed by C-terminal putative MCP domain together believed to act as a chemoreceptor, designated as Tlp7. This thesis describes that genes cj0952c and cj0951c are important for the invasion of host cells by the pathogen. In addition, it is clearly shown that both genes are not translated as one protein in $C$. jejuni isolate B2 contradicting the idea of a postulated read-through mechanism. Moreover, these genes are responsible for reduced motility of $C$. jejuni and alter the chemotactical behavior of the pathogen towards formic acid. Finally, growth curve analysis indicates that these genes are not related to the utilization of formic acid by formate dehydrogenase.

A second gene investigated in this thesis with regard to reduced infectivity of $C$. jejuni isolate B2 is cj0005c, which encodes a molybdopterin oxidoreductase. Together with the monohaem cytochrome $\mathrm{c}$ oxidoreductase subunit encoded by cj0004c, both subunits constitute a sulphite: cytochrome c oxidoreductase (SOR). 
Since $C$. jejuni lacks the glycolytic enzyme phosphofructokinase, glucose cannot be catabolized and other products serve as electron donors, e.g. succinate, malate, formate, D-lactate, hydrogen and $\mathrm{NAD}(\mathrm{P}) \mathrm{H}$. The possession of a sulphite oxidation system is believed to be important for $C$. jejuni to survive in food which has been treated with sulphite for preservative reasons, as well as in humans since it has been shown that sulphite is also released by neutrophils as part of the host defense. In this thesis SOR is docummented to be an important virulence factor of $C$. jejuni by reducing the motility of the pathogen and by the downregulation of $C$. jejuni genes known to belong to the virulence repertoire of the pathogen. 


\section{INTRODUCTION}

\subsection{Campylobacter jejuni}

The genus Campylobacter belongs to the epsilon proteobacteria class, order Campylobacterales and to the family Campylobacteriaceae, which consists of 16 species where Campylobacter jejuni is known as the most common causative agent of gastroenteritis. C. jejuni is Gram negative, curved or rod shaped with a size of 0.2 to $0.8 \mu \mathrm{m}$ and a single flagellum at one or both ends. An electron microscopy image of $C$. jejuni cells is shown in Fig 1.1.

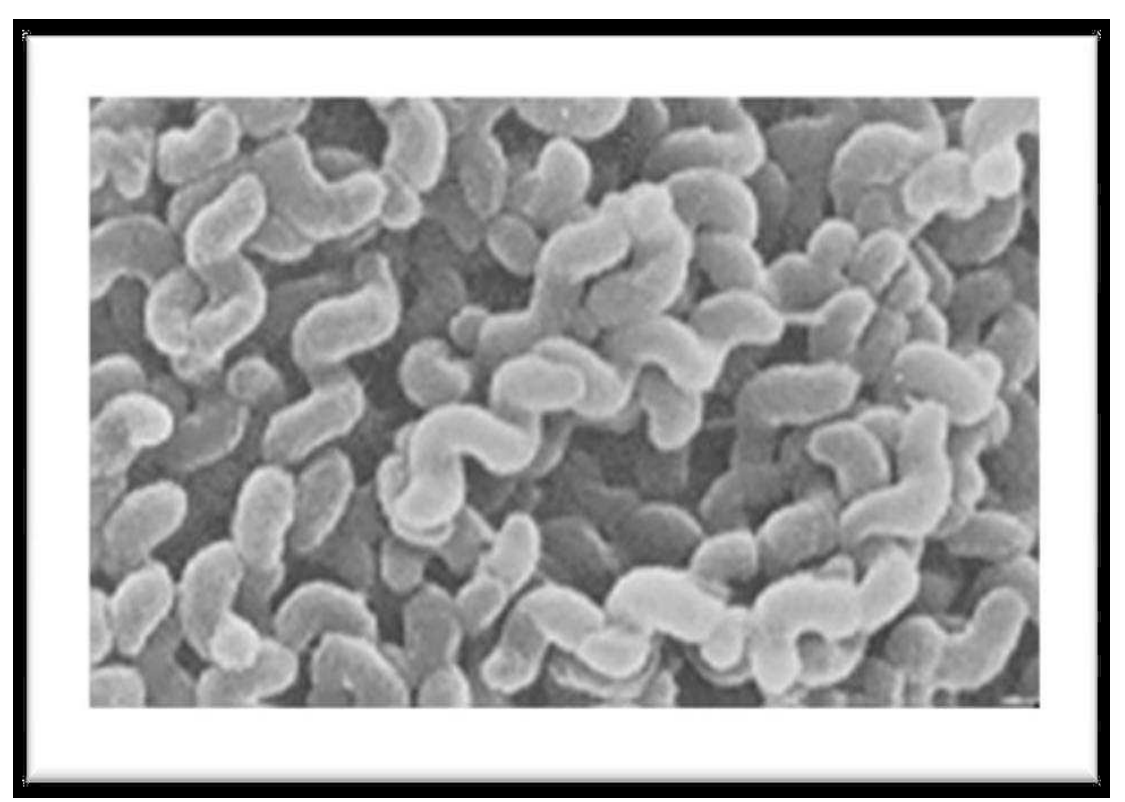

Figure 1.1 Electron microscopy image of $C$. jejuni cells

The figure reflects the typical spiral shape of $C$. jejuni bacteria (Courtesy: Prof. D. E. Taylor, University of Alberta, Canada and Adopted with permission from Lai-King N.G. et al., 1985; J. of Bacteriology 338-343)

The genome of $C$. jejuni has a size of approximately 1.6-1.7 Mbp and an AT ratio of about $70 \%$ (Parkhill et al., 2000). C. jejuni needs a special microaerophilic environment at $37-42{ }^{\circ} \mathrm{C}$ for its growth. Poultry is the most prominent reservoir for 
Campylobacter with microaerophilic conditions and a body temperature between 41 and $44{ }^{\circ} \mathrm{C}$. There are numerous transmission routes for Campylobacter spp. to infect humans. Most infections usually account through the consumption of undercooked contaminated poultry and, to a lesser extent, through the ingestion of contaminated meat from cattle, sheep, pigs, goats or seafood (Yamazaki et al., 2009; Whyte et al., 2004). The ecological cycle of $C$. jejuni is shown in Fig 1.2.

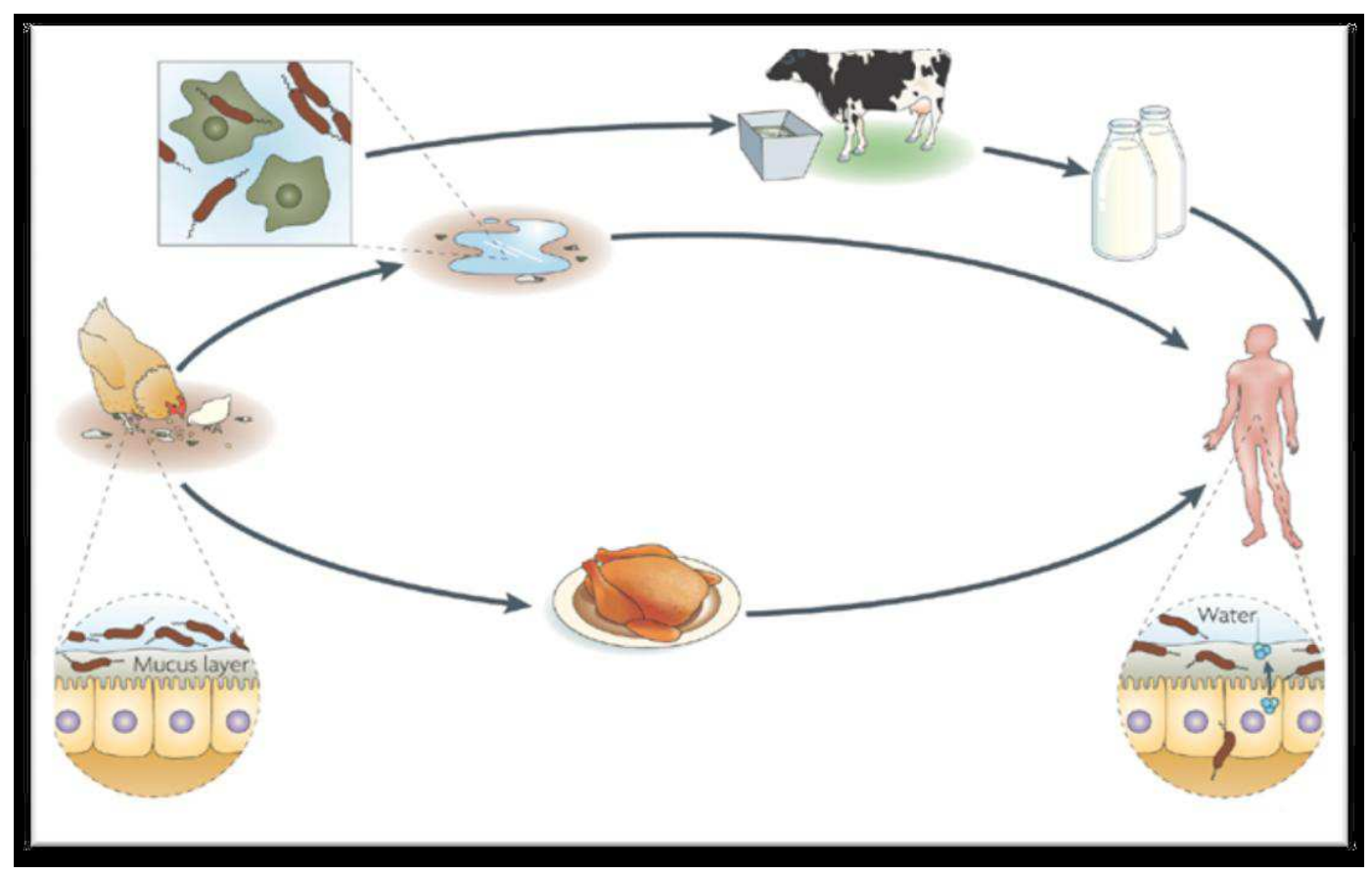

\section{Figure 1.2 The diagram of $C$. jejuni ecological cycle}

Ecological cycle shows that livestock, wild birds and chicken constitute the major reservoirs of $C$. jejuni. It colonizes the gastrointestinal mucosa of chicken; from there it is transferred between chicks within a flock through the faecal-oral route or enters the water supply. Humans are directly infected by $C$. jejuni through the consumption of contaminated animal products such as meat, particularly poultry or drinking of contaminated water or unpasteurized milk. In humans, $C$. jejuni invades the intestinal epithelial layer, which can result in inflammatory diarrhoea [Adopted with permission from Young et al., 2007; Nat Rev Microbio/ 5:665-679). 


\subsubsection{C. jejuni caused diseases}

Campylobacter jejuni is one of the most common causes of human diarrheal diseases. The incubation period can range from one to eight days with a typical time frame of three to four days (Skirrow et al., 1995). Clinical manifestation can range from watery to bloody diarrhoea often associated with acute abdominal cramps and fever. The peak of illness usually lasts for 24-48 hrs before it gradually resolves over a week, but convalescence may also be prolonged. The incidences of campylobacteriosis are increasing worldwide. Meanwhile, the number of cases often exceeding those of salmonellosis and shigellosis (Altekruse, 1999; Bryan and Doyle, 1995) with incidence rates in the United States of estimated 2.5 million cases and in Germany of more than 60,000 cases per year (Friedman et al., 2000). Age also determines the pattern of infection, the incidence under an age of four years is significantly higher than it is observed in the 19-29 year old patient group. There are peak incidences during the summer months indicating maximum replication rate of $C$. jejuni at higher temperatures (Asrat et al., 1999; Banmali et al., 2006).

The infection becomes subclinical, when patients frequently exposed to Campylobacter evolve immunity. This is most observed in children in developing countries, which are repeatedly infected, but also in certain western countries among people that are working in poultry abattoirs. In certain diseases e.g. diabetes, HIV infection, cancer in which the immune system is impaired, the risk of Campylobacter infection increases. In HIV positive patients, e.g. the risk of disease is 40 times higher than compared to normal patients (Sorvillo at al., 1990).

Like other enteropathogenic bacteria, C. jejuni can cause post-infectious manifestations like urticaria, erythemanodosum and reactive arthritis. In addition, C. jejuni has been recently described to be associated with immunoproliferative small intestinal diseases (Lecuit, 2004). The Guillain-Barré syndrome (GBS) is considered to be the most important post-infectious manifestation with reported 
incidences of 1.2 to 1.9 per 100,000 in Europe alone (Rees, 1998; Govoni, 2001). Increasing rates of GBS are reported with advancing age of 75 years of about four per 100,000 compared to less than one per 100,000 in patients younger than 18 to 30 years. The most common subtypes are acute motor axonal neuropathy (AMAN), acute inflammatory demyelinating polyradiculoneuropathy (AIDP) and when sensory fibers are affected, acute motor and sensory axonal neuropathy (AMSAN) (Asbury et al., 1969; McKhann et al., 1991; Griffin, 1996). The main symptoms of GBS are rapidly progressing bilateral and relative symmetrical weakness of the limbs and impairment of the respiratory muscles. Frequently, pain occurs from mild to severe (Asbury and Cornblath, 1990).

A reduction of the infection rate of $C$. jejuni in humans will be related to the knowledge of biological aspects and, in particular, to the knowledge of virulence factors, which are directly responsible to the pathogenesis of the disease. However, less is known about the pathogenesis of the disease at the molecular level. Meanwhile, genome sequences of different Campylobacter strains are available, which open new directions in Campylobacter research (Parkhill et al., 2000).

\subsection{General features of virulence associated factors of $C$. jajuni}

\subsubsection{Flagellum}

Campylobacter jejuni possesses a polar flagellum which mediates motility that, in turn, plays a very vital role for host colonization and host-cell invasion (Yao et al., 1994; Ormonde et al., 2000). Early studies indicated that aflagellated C. jejuni mutants illustrate clearly reduced internalization into the host cells in-vitro (Yao et al., 1994; Wassenaar and Blaser, 1999). The flagellum of Campylobacter jejuni is composed of O-linked glycosylated flagellin. It was observed that for the expression of a functional flagellum different strains of $C$. jejuni are possessing different numbers of genes, e.g. 50 (cj1293 to cj1342) adjacent to flaA and flaB, in case of $C$. jejuni strain NCTC 11168 (Parkhill, 2000). In contrast, in C. jejuni strain 
81-176, the flagellin O-linked glycosylation locus comprises only 26 genes (Guerry, 2006; Goon et al., 2003). Recent studies have suggested a twocomponent system comprised of the sensor FlgS and the response regulator FlgR to be the major control on flagella expression in $C$. jejuni. The composition of the cell wall and the flagellum of $C$. jejuni is shown in Fig. 1.3.

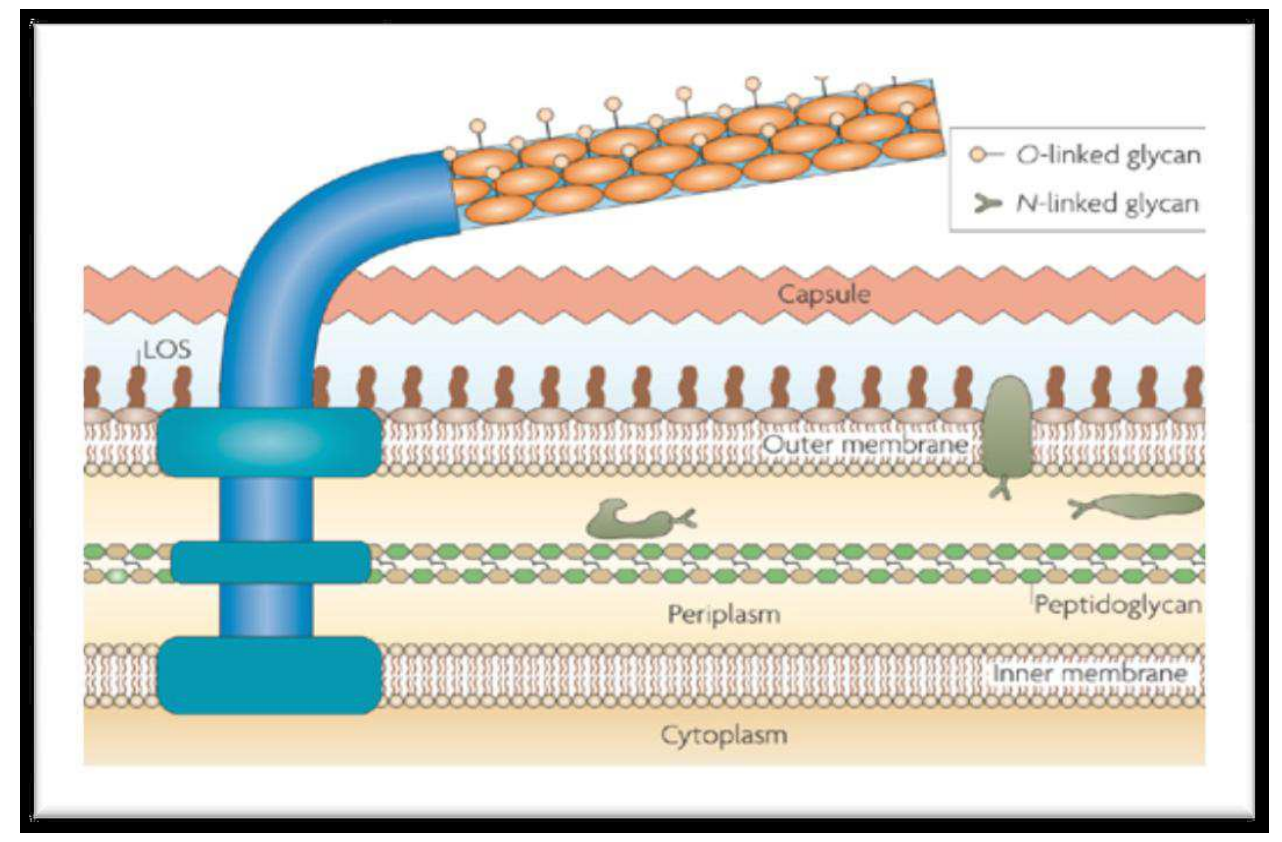

Figure 1.3 A cross-sectional appearance of the $C$. jejuni cell wall

The figure illustrates the flagellum, outer membrane protein and its associated lipooligosaccharides, capsule and the $\mathrm{O}$ - and $\mathrm{N}$-linked glycans [Adopted with permission from Young et al., 2007; Nat Rev Microbiol 5:665-679].

Specialized type-III-secretion systems have been shown to be important for virulence of many Gram-negative enteric pathogens. However, genomic studies have indicated that $C$. jejuni strains lack these systems (Galan and Collmer, 1999). However, there are several reports that the flagellum can function to secrete virulence associated proteins. One of these proteins that were identified along with seven other proteins is $\mathrm{CiaB}$ (Konkel et al., 1999). A ciaB mutant of $C$. 
jejuni strain F38011 has no obvious motility defects, but is unable to secrete any of the Cia proteins. Moreover, the mutant has a 50-fold reduced invasion capacity compared to the parental strain F38011 (Konkel et al., 1999). In contrast, a mutation of ciaB in $C$. jejuni strain 81-176 had no significant effect on the invasion of INT407 cells (Goon et al., 2006). These different observations might be due to variable invasion mechanisms among strains, cell lines used or methodologies (Goon et al., 2006). At least, a minimum flagella structure is required for the secretion of Cia proteins. In an flhB mutant secretion of the Cia proteins was not detected.

Recent studies have suggested that in both, in a fla $A$ mutant and in a flaB mutant secretion occurs, but not in a double mutant that lacks all filament structure (Konkel et al., 2004). Recently, secreted proteins FlgP and FlgQ, have been described to be important for flagellar motility in $C$. jejuni but their functional mechanisms remain unclear. Furthermore, they show no similarity with already characterized proteins (Sommerlad and Hendrixson, 2007).

\subsubsection{Invasion}

The invasion capability of $C$. jejuni is an important virulence factor. Intracellular bacterial survival and replication has been observed extensively using mammalian cell tissue. Also, the results of intestinal biopsies of patients have demonstrated that $C$. jejuni invades the cells of the gut. Thereby, the ability of $C$. jejuni to invade host cell has been shown to be strain depended (Newell et al., 1985). Statistically, significant differences were observed in the ability of host cell invasion of $C$. jejuni strains from individuals with non inflammatory diarrhea versus individuals with colitis (Everest, 1992). In addition, it was observed that some $C$. jejuni strains show a very low level of invasion (Konkel et al., 1992), suggesting the use of different mechanisms for cell entry similar to those observed for different Chlamydia serovars (Byrne and Moulder, 1978; Clausen et al., 1997). In order to characterize the interaction of $C$. jejuni with host cells in vitro, invasion assays, largely based upon gentamicin protection, have been used to study extensively 
the invasiveness of $C$. jejuni in various cell lines including HeLa, HEp2, INT 407 and Caco-2 cells (Fauche're et al., 1986; Konkel and Joens, 1989; Wassenaar et al., 1991; Everest et al., 1992; Russell and Blake, 1994). Furthermore, Russell et al., (1993) reported based on an experimental primate model of Macaca mulatta the ability of $C$. jejuni to invade colonic epithelial cells and to cause diarrhea. Moreover, mucosal damage as a result of a $C$. jejuni invasion of colonic epithelial cells has been reported in different animal models.

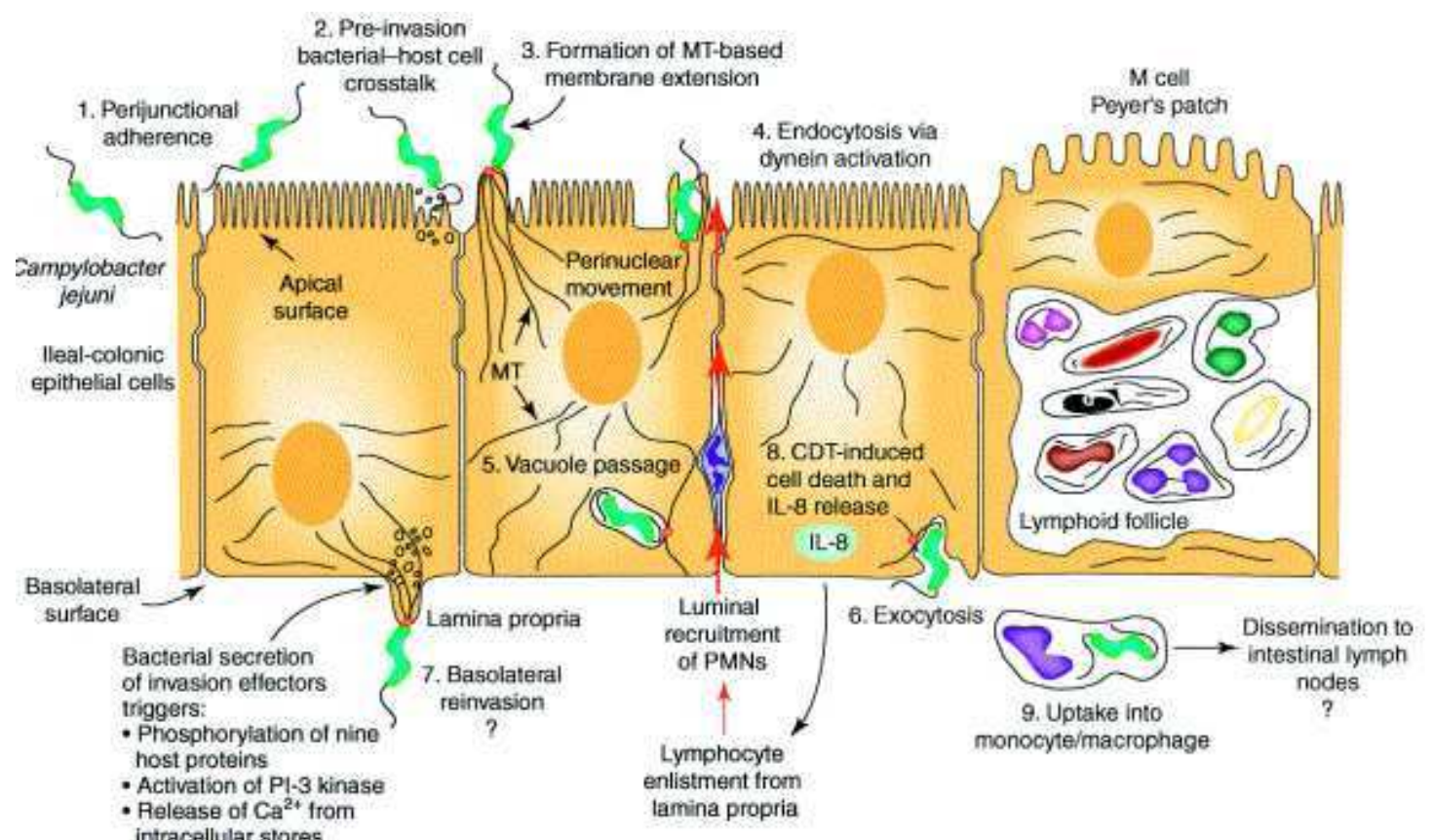

Figure 1.4 Pathogenesis model of $C$. jejuni.

(1) Initially, C. jejuni adheres at the apical cell surface at the peri-junctional region. (2) Where it secretes putative invasion effectors into the host cell. (3) Disruption of cortical actin filaments and an extension of microtubules (MTs) are mediated by host signalling cascades to form a membrane protrusion. (4) Endocytosis of the bacterium via membrane invagination. (5) Membrane bound $C$. jejuni vacuole moves to the basolateral surface through dynein along MTs. (6) For exocytosis (7) Again re-enter the epithelium basolaterally. (8) Interleukin (IL)-8 secretions of infected cells, and subsequent proliferation of lymphocytes from the lamina propria. (9) Uptake of $C$. jejuni into macrophages and local dissemination [Adopted with permission from Kopecko, et al. 2001; TRENDS in Microbiology 9:389-396]. 


\subsubsection{Chemotaxis}

Chemotaxis is the ability of an organism to move toward or away from a chemical stimulus. Thereby, chemotaxis and cellular motility contribute to the colonization and virulence of pathogenic bacteria (Josenhans and Suerbaum, 2002). It is also reported that chemotactic mediated motility plays an important role in disease progression of virulent organisms, e.g. Vibrio cholerae (Lee et al., 2001). Several studies demonstrated the ability of $C$. jejuni to sense its external environment through chemical gradients, indicating that chemotaxis is an important virulence factor which plays a crucial role in the colonization and pathogenicity (Yao et al., 1997). C. jejuni was found to exhibit a number of chemoreceptors for the detection of chemoattractants and chemorepellents such as the amino acids L-glutamate, Laspartate, L-cysteine, and L-serine, the carbohydrate L-fucose, and the organic acids pyruvate, fumarate, succinate, malate, citrate and ketoglutarate (Hugdahl et al., 1988).

The attraction and repulsion of chemicals is sensed by trans-membrane methylaccepting chemotaxis proteins (MCPs) which transmit the information to the flagellum motor via the histidine kinase CheA and the response regulator CheY. $C$. jejuni strain NCTC 11168 possesses altogether two aerotaxis genes and ten genes for putative chemoreceptor molecules, designated Tlps for transducer-like proteins (Marchant et al., 2002). It has been reported that colonization in chickens and the invasion of human epithelial and chicken embryo cells are severely impaired in $C$. jejuni strains with mutations in $t / p 1, t / p 3$, docB, and docC (Christina et al., 2009). The importance of the chemosensory receptors of $C$. jejuni for virulence has also been described with a mutant lacking Tlp9 (Cj1189c or CetB), which shows invasion deficiency of human tissue culture cells (Golden and Acheson, 2002). Furthermore, Tlp10 (Cj0019) and Tlp4 (Cj0262C) were found to be necessary for wild-type colonization of the chick gastrointestinal tract (Hendrixson and DiRita, 2004). Altogether, although chemotaxis has been demonstrated to be important for C. jejuni (Hugdahl et al., 1988; Takata et al., 1992), the mechanistic source of the sensory control of motility is yet to be 
elucidated.

\subsubsection{Cytolethal distending toxin}

Protein toxins are a well defined source of bacterial virulence with specific effects on normal host cell function. Mostly, they act on membranes or interfere with intracellular signaling by binding with specific signaling proteins thereby facilitating the process of infection. Although the genome of $C$. jejuni has been sequenced, yet only a few potential virulence factors produced are known (Parkhill et al., 2000). One of these is the cytolethal distending toxin (CDT), which is produced by a number of Campylobacter species, including C. jejuni, C. lari, C. coli, C. fetus, and C. upsaliensis, (Johnson and Lior, 1988; Mooney et al., 2001). This toxin was observed to induce cell distension in different mammalian cells such as HeLa, Chinese hamster ovary ( $\mathrm{CHO})$, Caco2 cells and other, which is characterized by elongation, swelling and eventually cell death (Whitehouse et al., 1998; Shenker et al., 1999). However, it was observed that not all cell types were sensitive to CDT because NIH 3T3 fibroblasts and mouse Y-1 adrenal cells are not affected by toxin treatment (Cortes-Bratti et al., 1999; Johnson and Lior, 1988). CDT is a holotoxin complex, which comprises of three polypeptide subunits encoded by the adjacent linked genes $c d t A, c d t B$, and $c d t C$ (Smith and Bayles, 2006). The entire complex is required to show a maximal activity (Heywood et al., 2005). It has been documented that $\mathrm{CdtA}, \mathrm{CdtB}$, or $\mathrm{CdtC}$ have no toxic activity when applied to cells separately, but when these three proteins are combined, they interact together to form an active tripartite holotoxin which produces maximum cell toxicity (Lara-Tejero and Galan, 2000). How CDT causes pathogenesis in C. jejuni is still unclear, but the mechanism of action is known. The toxic component CdtB (Lara-Tejero and Galán, 2001) shows activity similar to the enzyme deoxyribonuclease (DNasel) (Lara-Tejero and Galan, 2000) which causes cell cycle arrest in the G2/M transition phase through blocking of CDC2 kinase, an enzyme known to be involved in mitosis entry (Pickett and Whitehouse, 1999). The entry of CdtB in the nucleus, which depends on an atypical nuclear localization signal, is important for the cytotoxic activity (McSweeney and Dreyfus, 
2004). It was observed that CDT acts as nuclease and cuts the double-stranded DNA of host cells (Hassane et al., 2001).

Incubation of HeLa cells with lysates from cdtB-deficient $C$. jejuni strains 81-176 and NCTC 11168 showed that the NCTC 11168 lysate was greatly attenuated, while 81-176 lysates retained toxic activity, suggesting CDT to be the principal, but not the only toxin of $C$. jejuni (Purdy et al., 2000). It was also observed that $C$. jejuni mutants lacking CDT are possessing the competence to colonize NF-kBdeficient mice, but are unable to cause gastroenteritis as it was observed for the wild type (Fox et al., 2004). Similar studies were also performed in Helicobacter hepaticus, where CDT mutants are showing the same results regarding to the colonization of the mouse gut (Young et al., 2004).

\subsubsection{Translocation}

Translocation is defined as the ability of bacteria to translocate from the gastrointestinal tract (GIT) to other internal organs (Berg, 1983). The competence to translocate across the cell barrier greatly differs among different pathogens. For instance, $S$. Typhi is able to migrate across a polarized monolayer which leads to cellular destruction and a complete loss of the monolayer integrity, whereas $S$. typhimurium causes significant less damage in the initial stage of the infection process (Kops et al., 1996). In order to infect the host, $C$. jejuni must cross the mechanical and immunological barriers of the GIT. In most organisms, the mucus membrane serves as the first line of defense which is 30 to $50 \mu \mathrm{m}$ thick and is considered to be the first point of contact between the host cell and $C$. jejuni (Rozee et al., 1982). However, C. jejuni utilizes several virulence factors such as motility, adhesion, as well as capsule synthesis to penetrate these barriers (McSweegan and Walker, 1986). In addition, translocation was also observed in a few strains of $C$. jejuni across an intact polarized Caco-2 cell monolayer (Everes et al., 1992). The exact mechanism by which $C$. jejuni translocates across polarized cells is still unclear, however, electron microscopic studies suggested that both, transcellular and paracellular routes exist to cross polarized epithelial 
cells (Konkel et al., 1992a). Translocation also occurs by disruption of the tight junction of adjacent cells which was achieved by disturbing the cellular cytoskeleton or by altering the specific proteins of tight junctions which are claudins, occludin, and junctional adhesion molecules (Schneeberger and Lynch, 2004; MacCallum et al., 2005). Recently, it was also observed that Campylobacter induces translocation of non-invasive gastrointestinal bacteria (Lisa et al., 2009). The migration of Campylobacter spp. to the mesenteric lymph nodes is important for the pathogenesis of enteric infections which was observed in $\mathrm{C} 3 \mathrm{H}$ or athymic and euthymic BALB/c germfree mice (Levine et al., 1983; FauchEre et al., 1985; Yrios and Balish, 1986).

Translocation is inhibited by chloramphenicol, which indicates that this process is linked to active bacteria (Konkel et al., 1992a). In addition, it was observed that butyrate, which is an bacterial metabolite found in the lumen of the large intestine protects Caco-2 monolayers from C. jejuni infection (Rechkemmer et al., 1988; Von et. al., 2008)

\subsection{Response to stress}

Campylobacter species lack many adaptive responses as compared to other food-borne pathogens and therefore are more prone to environmental stress. The genomic study of $C$. jejuni might be a sound tool to insight into pathogenicity, growth and survival mechanisms under different stressful conditions (Tauxe et al., 1992). Many studies have been attempted to focus on the involved mechanisms of $C$. jejuni survival at thermal stress i.e. above $42{ }^{\circ} \mathrm{C}$. Konkel and coworkers (1998) have found that at least twenty four proteins were upregulated when heat shocked was provided to the cell in the temperature range from $43{ }^{0} \mathrm{C}$ to $48{ }^{\circ} \mathrm{C}$. Under normal environmental conditions, some proteins behave as chaperones to ensure proper protein folding and also help to eliminate misfolded proteins (Konkel et al., 1998). Seventeen proteins that are either heat shock or chaperone homologues have been investigated in $C$. jejuni (Stintzi, 2003). Among these heatshock proteins, some were molecular chaperones including GroELS, DnaJ and 
DnaK (Murphy et al., 2006). DnaJ has been further characterized by Konkel et al., (1998). Thereby, it has been established that the growth of DnaJ mutants was drastically reduced when incubated at $46{ }^{\circ} \mathrm{C}$. Furthermore, they have lost the ability to colonize chicken cells, which documents the fact that heat shock proteins have a key role in both, thermotolerance and colonization. Most prokaryotes possess two-component regulators, which act as a survival mechanism by affecting gene expression in response to an environmental change (Murphy et al., 2006). They are constituted by a cytoplasmic sensory histidine kinase and a response regulator in the cytoplasmic membrane.

There are nine response regulators and six histidine kinases that have been reported in C. jejuni (Murphy et al., 2006). A signal transduction system called RacR-RacS was described by Bras and coworkers (1999). To define temperature as the function of the two-component regulator, $C$. jejuni mutants were generated that do not produce RacR. RacR mutant bacterial cells show a growth rate as the normal strain at $37{ }^{\circ} \mathrm{C}$, but have declined growth at $42{ }^{\circ} \mathrm{C}$. The study concluded that RacR has a temperature-dependent influence on the growth rate. It has been demonstrated that $C$. jejuni cells in a stationary phase are less prone to heat stress as compared to the exponential-growth phase, suggesting that thermotolerance is not responsible by changes accompanying stationary phase entry (Kelly, 2001). This finding is further supported by the lack of the rpoS gene in the genome of $C$. jejuni (Parkhill et al., 2000).

The $\mathrm{pH}$-value is also a determining factor of bacterial growth. The optimum $\mathrm{pH}$ for C. jejuni growth and survival is in the range between 6.5 and 7.5 , with a maximum $\mathrm{pH}$ tolerance of about 9.0 (Chaveerach et al., 2003). C. jejuni cell numbers are significantly reduced when the $\mathrm{pH}$ is in a range above 9.0 or below 4.0 (Chaveerach et al., 2003). The survival and infectivity of $C$. jejuni depends how rapidly it adapts to the acidic environment in the gastrointestinal tract of the host (Reid et al., 2008). However, the mechanism of how $C$. jejuni responds to low $\mathrm{pH}$ values has not been well documented. C. jejuni strain Cl120 has an adaptive 
tolerance response (ATR) for survival in the acidic environment that is induced by acid and oxygen (Reid et al., 2008). Protein synthesis is essential to augment ATR, indicating that $\mathrm{pH}$-stimulating proteins are involved (Reid et al., 2008). However, a particular protein of $C$. jejuni responsible for protection against low $\mathrm{pH}$ has yet to be determined. (Reid et al., 2008, Ma et al., 2009).

Campylobacter spp. is less tolerant to a sudden change in solute concentrations (osmotic stress) than other food-borne pathogens (Doyle and Roman, 1982). Birk and coworkers (2004) used chicken juice to study $C$. jejuni survival at different sodium chloride concentrations. They reported that $C$. jejuni strains incubated at $42{ }^{\circ} \mathrm{C}$ were unable to grow in the presence of $2.0 \%$ sodium chloride. In addition, Campylobacter uses aerotaxis and chemotaxis as a defense mechanism to fight against various environmental responses (Hazeleger et al., 1998). 


\section{Objective of the study}

Campylobacter jejuni has emerged as an important food-born gastrointestinal pathogen for the last few decades in developing and industrialized countries. Furthermore, post infectious manifestations e.g. reactive arthritis or Guillain-barre syndrome might occur. In Germany approximately sixty thousand cases of Campylobacter mediated enteritis are reported annually. Although, a few factors that are essential for our understanding of the pathogenesis have been described, the mechanism of Campylobacter infection in humans is still not fully understood.

In order to identify pathogenesis-related genes that have not been described so far, an existing transposon-generated mutant library of Campylobacter jejuni should be screened to detect mutants with reduced capacity to invade host cells. Corresponding genes, identified by this method, should furthermore be characterized with regard to their biological function to explain the particular effect of these genes during the procedure of host cell infection. For this, specific assays to answer the question which step in the complicated process of attachment, entry and maintenance of intracellular survival is affected by these new virulence associated factors will be performed. Taken together, this study is expected to give new insights in Campylobacter pathogenesis by the identification and characterization of to date unfamiliar infection-related factors. 


\section{MATERIALS AND METHODS}

\subsection{Materials}

\subsubsection{Instruments}

\section{Instrument Model and Manufacturer}

Agarose gel electrophoresis cells

Keutz Labortechnik, Reiskirchen

Bio safety cabin

BDK and Luft Reinraumtechnik, Gmbh

Balances

BP 221 S, Sartorius, Göttingen

LP6200 S, Sartorius, Göttingen

Cell culture microscope

Modell DM IL, Leica, Heidelberg

Centrifuges

Megafuge 2.0 RS, Heraeus, Hanau

Megafuge 2.0, Heraeus, Hanau

Modell 5417 R, Eppendorf, Hamburg

Modell 5417 C, Eppendorf, Hamburg

RC-26 Plus, Sorvall-Kendro, Hanau

Electro Cell Manipulator

BTX ECM600, San Deigo, California

Incubators

Heraeus, Hanau

Gel documentation system

BioDoc II, Biometra, Göttingen

Gel electrophoresis cells for PAGE Modell

Mini Protean II, BioRad, München

Lightcycler 1.5

Roche, Basel, Schweiz

Magnet bars

RCT basic, IKA Labortechnik, Staufen

pH-Meter

Modell 766 Calimatic, Knick, Berlin

Power supply

EPS 600, Pharmacia Biotech

Freiburg Standard Power Pack P25,

Biometra,Göttigen

Pipet aid

Hirschmann Laborgeräte, Eberstadt

Pipettes

Modell Research, Eppendorf,

Hamburg 


$\begin{array}{ll}\text { Photometer } & \text { Ultraspec 1000, Pharmacia Biotech, } \\ & \text { Freiburg } \\ \text { Shaking Incubater } & \text { SM-30 Control, Johanna Otto GmbH, } \\ \text { Thermomixer } & \text { Modell Compact, Eppendorf, } \\ & \text { Hamburg } \\ \text { Thermocycler } & \text { Modell T3, Biometra, Göttingen } \\ \text { UV-Crosslinker } & \text { UVC-500, Hoefer, San Francisco, } \\ & \text { CA, USA } \\ \text { UV-Table } & \text { TFX-20M, Gibco BRL, Eggenstein } \\ \text { Water bath } & \text { Laborte Chink, Hannover Vinnhorst } \\ \text { NanoDrop ND } 1000 \text { spectrophotometer } & \text { Peqlab, Erlangen }\end{array}$

\subsubsection{Cell culture media and additives}

\subsubsection{Antibiotics (disc)}

Ampicillin

Kanamycin

Tetracycline

Ciprofloxacin

Gentamicin

Chloramphenicol

\subsubsection{Antibiotics (powder)}

Chloramphenicol

Kanamycin

Penicillin
Oxoid Hampshire, England

Oxoid Hampshire, England

Oxoid Hampshire, England

Oxoid Hampshire, England

Oxoid Hampshire, England

Oxoid Hampshire, England

Sigma Steinheim Germany

Sigma Steinheim Germany

Sigma Steinheim Germany

\subsubsection{Media and additives}

Dulbecco's MEM (DMEM) Medium supplemented with 580 mg/l L-glutamine und $3.7 \mathrm{~g} / \mathrm{l} \mathrm{NaHCO}$.

DMEM Medium (10x) with $4.5 \mathrm{~g} / \mathrm{l}$ D-glucose, $8 \mathrm{mg} / \mathrm{l}$ Phenol Red, without $\mathrm{NaHCO}_{3}$, Na-pyruvate and L-glutamine. 
Penicillin / Streptomycin, 10,000 U / 10,000 $\mu \mathrm{g} / \mathrm{ml}$.

Fetal Calf Serum (FCS); heat inactivated by incubation for $45 \mathrm{~min}$ at $56{ }^{\circ} \mathrm{C}$.

EDTA (Versen) $1 \%$ in PBS without $\mathrm{Ca}^{2+}, \mathrm{Mg}^{2+}$

Trypsin (1:250) $0.25 \%$ in PBS without $\mathrm{Ca}^{2+}, \mathrm{Mg}^{2+}$

PBS (phosphate buffered saline)

All the product mentioned above were obtain from Biochrom, Berlin

\subsubsection{Disposable materials and plastic ware}

Disposable materials and plasticware used in the cell culture were purchased from Nunc,

Roskilde, Denmark; Falcon, Becton - Dickinson, Heidelberg; Corning Costar, Bodenheim; Greiner, Frickenhausen; Braun, Braun-Melsungen, Melsungen.

\subsubsection{Cell lines}

Human colon carcinoma Caco2

\subsubsection{Bacterial strains and mutants}

\begin{tabular}{|c|c|c|}
\hline Bacterial Strains & Specification & Source \\
\hline \multirow[t]{3}{*}{ Campylobacter jejuni } & NCTC11168 & Dr. A. Karlyshev \\
\hline & $81-176$ & Prof. M. Kist, Freiburg \\
\hline & B2 & $\begin{array}{l}\text { University Medical Center, } \\
\text { Göttingen }\end{array}$ \\
\hline \multirow[t]{2}{*}{ E. coli } & $\mathrm{DH} 5 \alpha$ & Invitrogen \\
\hline & BL21 & CodonPlus-RIL Stratagene \\
\hline $\begin{array}{l}\text { Transposone-generated mutants } \\
\text { Library }\end{array}$ & B2 mutants & $\begin{array}{l}\text { Dr. J. I. Dasti, Gottingen } \\
\text { Institute of Medical. } \\
\text { Microbiology }\end{array}$ \\
\hline
\end{tabular}

\subsubsection{Bacterial culture media}

\section{Luria Bertani (LB) Broth}

1\% Bacto-Trypton

0.5\% Bacto-yeast extract

$0.5 \% \mathrm{NaCl}$ 
LB-Plates

Brain Heart Infusion

Columbia Agar Base

Muller Hinton Agar

Sheep Blood
0.5\% Bacto-yeast extract

$0.1 \%$ Bacto-Trypton

$0.5 \% \mathrm{NaCl}$

$1.5 \%$ Agar (Difco, Detroit, USA)

Bacto $^{T M}$

Merck, Darmstadt

Oxoid, Wesel

Oxoid Wesel

\subsubsection{Chemicals and reagents}

Chemicals and solutions were purchased from Roche (Mannheim), Merck (Darmstadt), Roth (Karlsruhe), Calbiochem (Bad Soden) or Sigma (Deisenhofen). In general, solutions were prepared using double distilled water.

\subsubsection{Antibodies}

Monoclonal Mouse anti His Tag primary antibody (Qiagen) Hilden Horseradish-Peroxidase-Conjugated anti Mouse secondary antibody (Dianova)

\subsubsection{Membranes and filters}

Nitrocellulose membrane

Hybond N (Amersham Biosciences)

Nylon membrane Hybond P (Amersham Biosciences)

Filter discs Oxoid, Wesel

Corning $26 \mathrm{~mm}$ Syringe Filter, $0.20 \mu \mathrm{m}$ Micron Membrane, $0.45 \mu \mathrm{m}$ Micron Membrane

\subsubsection{Enzymes}

Quick T4 DNA Ligase

Proteinase $\mathrm{K}$

Pfu DNA-Polymerase

Taq DNA-Polymerase
New England Biolabs

Roth, Karlsruhe

Promega, Mannheim

Roche, Mannheim 
Restriction endonucleases

Alkaline antarctic Phosphatase

KOD DNA-Polymerase

RNase A

DNase 1

RQI Rnase Free DNA
New England Biolabs

New England Biolabs

Novagen

Sigma-Aldrich, Steinheim

Sigma-Aldrich, Steinheim

Promega, Mannheim

\subsubsection{Standard Buffers}

Alkaline Phosphatase (AP) staining solution $0.05 \%$ (v/v) BCIP $0.5 \%(\mathrm{v} / \mathrm{v}) \mathrm{NBT}$

BCIP

Blocking solution

Coomassie staining solution

10x DNA loading dye

NBT

Separating gel (12\%)
$5 \%(w / v)$ 5-bromo-4-chloro-3-indolylphosphate in $\mathrm{dd}_{2} \mathrm{O}$

$5 \%(w / v)$ dry skimmed milk power

$0.2 \%(v / v)$ Tween 20

$0.2 \%(\mathrm{w} / \mathrm{v}) \mathrm{NaN}_{3}$

in $1 \times$ PBS (pH 7.4)

$0.025 \%(w / v)$ Coomassie brilliant

blue $\mathrm{G}$

$30 \%(\mathrm{v} / \mathrm{v})$ methanol

10\% (v/v) acetic acid

in $\mathrm{ddH}_{2} \mathrm{O}$

$30 \%(v / v)$ methanol

$10 \%(\mathrm{v} / \mathrm{v})$ acetic acid

in $\mathrm{ddH}_{2} \mathrm{O}$

$40 \%$ (v/v) glycerol

$1 \%(w / v)$ bromophenol blue

in TE buffer ( $\mathrm{pH} 8.0)$

$1 \%(\mathrm{w} / \mathrm{v})$ nitotetrazolium blue chloride in $\mathrm{ddH}_{2} \mathrm{O}$

$0.940 \mathrm{ml} 2 \mathrm{M}$ Tris- $\mathrm{HCl}(\mathrm{pH} 8.8)$

$0.1 \mathrm{ml} \mathrm{10 \% (w/v)} \mathrm{SDS}$ 
Stocking gel (4.4\%)

$1.94 \mathrm{ml} \mathrm{30 \%} \mathrm{acrylamide}$

$0.02 \mathrm{ml} 10 \%(\mathrm{w} / \mathrm{v})$ APS

$0.01 \mathrm{ml}$ TEMED

$2.0 \mathrm{ml} \mathrm{dd} \mathrm{H}_{2} \mathrm{O}$

$0.625 \mathrm{ml} 0.5 \mathrm{M}$ Tris-HCl (pH 6.8)

$0.05 \mathrm{ml} 10 \%$ (w/v) SDS

$0.36 \mathrm{ml} \mathrm{30 \%} \mathrm{acrylamide/}$

0,125 ml 10\% (w/v) APS

$0.01 \mathrm{ml}$ TEMED

$0.01 \mathrm{ml}$ Bromphenol Blue in $\mathrm{ddH}_{2} \mathrm{O}$

2x Sample buffer

$20 \%(v / v)$ glycerol

$1 \%(w / v)$ SDS

125 mM Tris- $\mathrm{HCl}$ (pH 6.8)

$0.002 \%(w / v)$ bromophenol blue 65 mM DTT

in $10 \mathrm{ml} \mathrm{ddH} \mathrm{H}_{2} \mathrm{O}$

Substrate solution

$200 \mathrm{ml} \mathrm{10 \%} \mathrm{(v/v)} \mathrm{diethamolamine}$ (pH 9.6)

20 ul $5 \mathrm{mM} \mathrm{MgCl}_{2}$

$800 \mathrm{ml}$ physiological $\mathrm{NaCl}(\mathrm{pH} 7.4)$

50x TAE (pH 8.0) $242 \mathrm{~g}$ Tris

$57.1 \mathrm{ml} \mathrm{98 \%} \mathrm{acetic} \mathrm{acid}$ $100 \mathrm{ml} 0.5$ M EDTA (pH 8.0)

in $1 \mathrm{~L} \mathrm{ddH} \mathrm{H}_{2} \mathrm{O}$

10x TE buffer (pH 8.0)

$100 \mathrm{mM}$ Tris

100 mM EDTA

in $\mathrm{ddH}_{2} \mathrm{O}$

Washing solution

0.05\% (v/v) Tween 20

in 1xTBS (pH7.4)

1\% Triton X-100/PBS

1\% (v/v) Triton X-100 
TFB1 (pH 5.8)

TFB2 (pH 6.8)

\subsubsection{Kits and reagents}

\section{RNA Isolation}

Ribo-Pure Bacteria Kit

Reverse Transcription

One Step RT-PCR Kit

DNA Cloning Kit

QIAGEN PCR Cloning Kit

Plasmid-DNA Isolation

GenElute Plasmid Mini Prep Kit

DNA Isolation from Agarose Gels

QIAEX II Gel Extraction Kit in $1 \times$ PBS

$100 \mathrm{mM} \mathrm{RbCl}$

$50 \mathrm{mM} \mathrm{MnCl}{ }_{2}$

$30 \mathrm{mM} \mathrm{KAc}$

$10 \mathrm{mM} \mathrm{CaCl}_{2}$

$15 \%(\mathrm{v} / \mathrm{v})$ glycerol

in $\mathrm{ddH}_{2} \mathrm{O}$

$10 \mathrm{mM}$ MOPS

$10 \mathrm{mM} \mathrm{RbCl}$

$75 \mathrm{mM} \mathrm{CaCl}_{2}$

$15 \%(v / v)$ glycerol

in $\mathrm{ddH}_{2} \mathrm{O}$

Ambion

Qiagen, Hilden

Qiagen, Hilden

Sigma-Aldrich, Deisenhofen

Qiagen, Hilden 
DNA and PCR Purification

QIAquick PCR Purification Kit Qiagen, Hilden

Genomic DNA Isolation

QIAamp. DNA Mini Kit

Qiagen, Hilden

Protein-His Tag Expression and Purification

QIAexpress Kit

Qiagen, Hilden

Quantification of Proteins

Pierce BCA Protein Assay Kit

Pierce, Rockford, IL, USA

\section{Molecular weight markers}

GeneRuler 100 bp DNA Ladder

MBI Fermentas

GeneRuler 1kb DNA Ladder

MBI Fermentas

Prestained Protein Marker, Broad Range

New England Biolabs 


\subsubsection{Oligonucleotide}

Table 2.1 HPLC-purified oligonucleotide primers used for sequencing

\begin{tabular}{lll}
\hline Gene & Primer name & Sequence (5' to 3') \\
\hline \multirow{2}{*}{ aphA-3 } & KanF & TATCACCTCAAATGGTTCGCT \\
cj0952c-51c & Cj0952c-51cF & GCCAATTCATTTTCATTTTGAGA \\
& Cj0952c-51cR & TTTTAATGCTTAAAGTTGTTGTTGG
\end{tabular}

Table 2.2 HPLC-purified oligonucleotide primers used for cloning in pRRC and pBluescript II

\begin{tabular}{|c|c|c|}
\hline Gene & Primer name & Sequence (5' to $\left.3^{\prime}\right)$ \\
\hline \multirow[t]{2}{*}{ cj0952c } & Cj0952cF & GCTCTAGATAGGAAACTATGATGTTTAAAACTA \\
\hline & Cj0952cR & GCTCTAGAAATTAATTGATATATCCACA \\
\hline cj0952c/ & Cj0952cF & GCTCTAGATAGGAAACTATGATGTTTAAAACTA \\
\hline cj0951c & Cj0951cR & NNNTCTAGACCTTTAAATTTGAAATTGGTTAAGTTCGC \\
\hline cj0952c/ & Cj0952cF & GCTCTAGATAGGAAACTATGATGTTTAAAACTA \\
\hline \multirow[t]{2}{*}{ cj0951cHis } & Cj0951His & GGCTCTAGATTAGTGATGGTGATGGTGATGAATTTG \\
\hline & & AAATTGGTTAAGTTCGC \\
\hline cj0952c/ & Cj0952F & GCTCTAGATAGGAAACTATGATGTTTAAAACTA \\
\hline cj0950c & Cj0950R & NNNTCTAGAGGGCATTATTTAAAAAAGATTTTGATTTC \\
\hline \multirow[t]{2}{*}{ cj0005c } & Cj00005cF & GCTCTAGATTAAGGATATTTCAATGAAACAGAAC \\
\hline & Cj00005cR & GCTCTAGAACTTAAACTCCATAAACATTAACTC \\
\hline \multirow[t]{2}{*}{ kanR } & Kan1 & P-GTAAGATTATACCGAGGTATGAAAACG \\
\hline & Kan2 & P-AATCTAGGTACTAAAACAATTCATCCA \\
\hline
\end{tabular}

Xbal restriction sites for cloning in pRRC and pBluescript II are underlined, the start codon of cj0952c and cj0005c are shown in bold and the putative SD sequence is illustrated in italic. Primers Kan1 and Kan2 are 5'-phosphorylated. Gene numbering according to $C$. jejuni strain NCTC 11168. 
Table 2.3 Oligonucleotide primers used for semiquantitative real-time RT-PCR analysis

\begin{tabular}{|c|c|c|}
\hline Gene & Primer name & Sequence (5' to $\left.3^{\prime}\right)$ \\
\hline \multirow[t]{2}{*}{$23 S$} & Cj23SF & GTTCGCCATTTAAAGCGGTA \\
\hline & Cj23SR & TGCTCTTGGCAGAACAACAG \\
\hline \multirow[t]{2}{*}{ cj0951c } & Cj0951fF & GAGTTACCAAAGCCCTAGCATC \\
\hline & Cj0951R & CATGGTCAATCAAGCAGG \\
\hline \multirow[t]{2}{*}{ cj0952c } & Cj0952F & GCCACTTCCTTGAGC \\
\hline & Cj0952R & CGCCCTTTGATTGCAGATG \\
\hline \multirow[t]{2}{*}{ cj0953c } & Cj0953F & GGCTGAAATGATTACTTCTAC \\
\hline & Cj0953R & CTAACCGATATTAACGCAGC \\
\hline \multirow[t]{2}{*}{ groEL (cj1221) } & GroELF & ATGGGGCCAAGAGGACGCAA \\
\hline & GroELR & GCAGTAGTTGTTCCATCGCCTGCT \\
\hline \multirow[t]{2}{*}{ dnaJ (cj1260c) } & DnaJF & GGCTTTGGCTCATCGCGTCG \\
\hline & DnaJR & ACCTTGAGAAACCCCAACCTGTCC \\
\hline \multirow[t]{2}{*}{ dnaK (cj0759) } & DnaKF & TGCTGTGTATGAACGCGGCGA \\
\hline & DnaKR & ACCGCTTGGCGTTTTGCACT \\
\hline \multirow[t]{2}{*}{$\operatorname{cheY}(\operatorname{cj1118c)}$} & CheYF & GCTGAGCATGGCGTTGAAGC \\
\hline & CheYR & АCTCCAAGCCATTCATTTCTGGCA \\
\hline \multirow[t]{2}{*}{ t/p7 (cj0952c) } & Tlp7F & GAGTTACCAAAGCCCTAGCATC \\
\hline & Tlp7R & CATGGTCAATCAAGCAGG \\
\hline \multirow[t]{2}{*}{$\operatorname{cadF}(c j 1478 c)$} & CadFF & TGGACATTATGGCGCGGGTGT \\
\hline & CadFR & TGTGGAGTTGCACGAGTATCAGCA \\
\hline \multirow[t]{2}{*}{ peb1a (cj0921c) } & Peb1aF & ACAAGAGGCCCTTTGCTTGATAATGGT \\
\hline & Peb1aR & AGTTGCAGCTTGAGCCACTCCA \\
\hline \multirow[t]{2}{*}{ jlpA (cj0983) } & JlpAF & AGCACACAGGGAATCGACAGCA \\
\hline & JlpAR & AAATGACGCTCCGCCCATTAACA \\
\hline \multirow[t]{2}{*}{$\operatorname{ciaB}(c j 0914 c)$} & CiaBF & TCATGCGGTGGCATTAGAATGGG \\
\hline & CiaBR & CATCATTTGGAACGACTTGAGCTGAGA \\
\hline \multirow[t]{2}{*}{$c d t B(c j 0078 c)$} & CdtBF & TGCAAGGCTCATCCGCAGCC \\
\hline & CdtBR & TGGCGTCCTGTTGGAGTGGC \\
\hline
\end{tabular}




\begin{tabular}{|c|c|c|}
\hline \multirow[t]{2}{*}{ flaA (cj1339c) } & FlaAF & AGGCGCTATGGCTGTGATGGA \\
\hline & FlaAR & TGCACTCTCGGCTGCAAAGTCT \\
\hline \multirow[t]{2}{*}{ ptmC (cj1327) } & PtmCF & ACAGGCATGGAAATTTAGGCGA \\
\hline & PtmCR & ACAAGCTCCCAAGGCAACCGC \\
\hline \multirow[t]{2}{*}{ ptmD (cj1328) } & PtmDF & ACCAAAGCAAATGCCGATGAAAATGG \\
\hline & PtmDR & AACGCCCTTTTTGTCTATCGCCT \\
\hline \multirow[t]{2}{*}{ ptmE (cj1329) } & PtmEF & ACTAGGTACAGCAGGGGCTTTAAGC \\
\hline & PtmER & CTCACGCACGCAAACGCTCA \\
\hline \multirow[t]{2}{*}{ ptmF (cj1330) } & PtmFF & GCTAGGTGGTGGGGTTTTACTCGA \\
\hline & PtmFR & GCCAAAAAGGCAAAATCATCGCTTGT \\
\hline \multirow[t]{2}{*}{$p t m B(c j 1331)$} & PtmBF & GCTAGGGGTGGTAGCAAAGGCG \\
\hline & PtmBR & GCAAGGCATCACGCATGACAGGA \\
\hline \multirow[t]{2}{*}{ ptmA (cj1332) } & PtmAF & CGCGTTAATACTCTAGCAAGCGGAGG \\
\hline & PtmAR & AAGCCCCATCCATCATCTACCACT \\
\hline \multirow[t]{2}{*}{ pseD (cj1333) } & PseDF & CCCATGGCAATGATCCTCTTGATGC \\
\hline & PseDR & TCCAAAGAAAGCACATAATCGGGCT \\
\hline \multirow[t]{2}{*}{ pseE (cj1337) } & PseEF & TGTTGGAACGCGATGAAATAGTAGCT \\
\hline & PseER & GCATCTCGCAAAAGGCTGGCC \\
\hline \multirow[t]{2}{*}{ pseB (cj1293) } & PseBF & CGGGCGGAACAGGCTCGTTT \\
\hline & PseBR & CGTCGATGACATTTTGCGCACCG \\
\hline \multirow[t]{2}{*}{ pseC (cj1294) } & PseCF & GCCGCTACAGCTAATGCGGC \\
\hline & PseCR & GCTGGTTTATCCCTAAAGCACAAGCA \\
\hline \multirow[t]{2}{*}{ pgIE (cj1121c) } & PglEF & AGGAGCTATTGGCGTAGCGCA \\
\hline & PglER & TCATCGCCGTGCCACTTGC \\
\hline \multirow[t]{2}{*}{ pseA (cj1316) } & PseAF & ATGGCGGTCCTGCGAGTAGT \\
\hline & PseAR & TGCTCTAACCAAAGCCGTATTTGCA \\
\hline \multirow[t]{2}{*}{ porA (cj1259) } & PorAF & TGGCTGCAGAGCAAGGTGCA \\
\hline & PorAR & AACCTACAGCAGCAGCACCG \\
\hline \multirow[t]{2}{*}{ flap (cj1279c) } & FlpAF & GGTTCTGAACGAAGCCCGGCT \\
\hline & FlpAR & AGACGCTATGGCGGGGGAGCA \\
\hline
\end{tabular}




$\begin{array}{lll}p t m G(c j 1324) & \text { PtmGF } & \text { TGTGATCACTGCGGTGATGCCAA } \\ & \text { PtmGR } & \text { ACCTCCACTTACGGCAATAGCACA } \\ \text { ptmH }(c j 1325) & \text { PtmHF } & \text { TGGCAATGGTGTACATTCAGCG } \\ & \text { PtmHR } & \text { TCCACCTGTGTTCAAAAGTTCGT }\end{array}$




\subsection{Methods}

\subsubsection{Cell Culture}

\subsubsection{Bacterial strains and culture conditions}

The Campylobacter jejuni strains used in this study were B2, isolated in the University Medical Center Göttingen from a patient suffering from gastroenteritis (Schmidt-Ott et al., 2005; Dasti et al., 2007), NCTC 11168 and strain 81-176. All strains were cultured on Columbia blood agar supplemented with $5 \%$ defibrinated sheep blood under microaerophilic conditions (85\% $\mathrm{N}_{2}, 10 \% \mathrm{CO}_{2}$, and $5 \% \mathrm{O}_{2}$ ) at $42{ }^{\circ} \mathrm{C}$ for 24 hours. As per requirement, appropriate antibiotics were added at the following concentrations: kanamycin $(50 \mu \mathrm{g} / \mathrm{ml})$ or chloramphenicol $(30 \mu \mathrm{g} / \mathrm{ml})$. For growth experiments, bacteria were grown at $42{ }^{0} \mathrm{C}$ in $\mathrm{MH}$ broth under microaerophilic conditions in the presence or absence of $10 \mathrm{mM}$ formic acid or 10 $\mathrm{mM} \mathrm{Na}_{2} \mathrm{SO}_{3}$. Every growth experiment was carried out three times. Escherichia coli strain DH5a which was used for cloning experiments was grown on LuriaBertani (LB) agar or in Luria-Bertani broth at $37^{\circ} \mathrm{C}$. When necessary, the medium was supplemented with ampicillin $(100 \mu \mathrm{g} / \mathrm{ml})$.

\subsubsection{Preparation of chemical competent E.coli cells}

A single colony of E.coli DH5a was inoculated into $10 \mathrm{ml}$ of LB broth and kept overnight at $37{ }^{\circ} \mathrm{C}$ under shaking. $2 \mathrm{ml}$ of the over night culture was added into $100 \mathrm{ml}$ of pre-warmed LB broth and left on the shaker until an $\mathrm{OD}_{(600 \mathrm{~nm})}$ of 0.5 was reached. The culture was transferred into a $50 \mathrm{ml}$ Falcon tube and cooled on ice for $10 \mathrm{~min}$. Cells were centrifugated at $4000 \times \mathrm{g}$ for $10 \mathrm{~min}$ at $4{ }^{\circ} \mathrm{C}$ in a pre-cooled centrifuge. Then the cell pellet was gently resuspended in $30 \mathrm{ml}$ ice cold TFB1 buffer and incubated on ice for $30 \mathrm{~min}$. Cells were pelleted by centrifugation as described above, resuspended in $2 \mathrm{ml}$ ice-cold TFB2 buffer and incubated on ice for another $30 \mathrm{~min}$. After incubation aliquots of $100 \mu \mathrm{l}$ were stored at $-80{ }^{\circ} \mathrm{C}$. 


\subsubsection{Generation of electrocompetent bacterial cells}

$10 \mathrm{ml}$ of LB-broth were inoculated with a single $E$. coli DH5a colony and incubated overnight at $37{ }^{\circ} \mathrm{C}$ under shaking. Afterward, three $\mathrm{ml}$ of the overnight culture were grown in $500 \mathrm{ml}$ LB-broth at $37{ }^{\circ} \mathrm{C}$ until an $\mathrm{OD}_{(600 \mathrm{~nm})}$ of $0.35-0.45$ was reached. After placing the culture on ice for $10 \mathrm{~min}$ it was centrifuged for $15 \mathrm{~min}$ at $4,000 \times \mathrm{g}$ at $4{ }^{0} \mathrm{C}$. The pellet was gently resuspended in $50 \mathrm{ml}$ cold water and again centrifuged for $15 \mathrm{~min}$ at 4,000 $\times \mathrm{g}$. After repeating this step three times, the cell pellet was resuspended in $15 \mathrm{ml} 10 \%$ glycerol and centrifuged again. Finally, the cell pellet was carefully dissolved in a final volume of $1 \mathrm{ml} 10 \%$ glycerol and $100 \mu \mathrm{l}$ aliquots were used for each electroporation. In order to prepare competent cells of $C$. jejuni, cells were collected from Columbia blood agar plates and centrifuged at $5,000 \times \mathrm{g}$ at $4{ }^{0} \mathrm{C}$ for $10 \mathrm{~min}$. The cells so obtained were resuspended in $1 \mathrm{ml}$ ice-cold washing buffer containing $272 \mathrm{mM}$ sucrose and 15 $\%$ glycerol at $4{ }^{0} \mathrm{C}$. After repeating this step three times, the pellet was resuspended in $400 \mu \mathrm{l}$ washing buffer and $100 \mu \mathrm{l}$ aliquots were used for each transformation.

\subsubsection{Electroporation}

After the addition of 0.5 to $3 \mu \mathrm{g}$ of plasmid DNA to the electrocompetent cells, the mixture was transferred into an ice-cold electroporation cuvette and the cuvette was incubated on ice for $30 \mathrm{sec}$. Electroporation was performed at $2.5 \mathrm{kV}, 25 \mu \mathrm{F}$ and $200 \Omega$ using a BTX Electro Cell Manipulator. Then, $500 \mu \mathrm{l}$ of SOC medium was added to the cuvette and in case of $C$. jejuni the suspension was transferred onto a non selective Columbia blood agar plates and incubated overnight at $37{ }^{\circ} \mathrm{C}$ under microaerophilic conditions. Finally, the cells were transferred onto selective plates and incubated at $42{ }^{\circ} \mathrm{C}$ under microaerophilic conditions for further 2-3 days. After electroporation of E.coli cells, the suspension was transferred to a polypropylene tube, incubated with gentle rotation for 1 hour at $37{ }^{\circ} \mathrm{C}$ and plated on LB agar containing the appropriate antibiotic. 


\subsubsection{Cultivation of eukaryotic cells}

Human colon carcinoma 2 (Caco2) cells were maintained in $175 \mathrm{~cm}^{2}$ and $25 \mathrm{~cm}^{2}$ cell culture flasks as well as in 6 well plates in Dulbecco minimal essential medium (DMEM) supplemented with $10 \%$ heat inactivated fetal calf serum, $1 \mathrm{x}$ non-essential amino acids, with/without $100 \mathrm{U} / \mathrm{ml}$ penicillin, and $100 \mu \mathrm{g} / \mathrm{ml}$ streptomycin. The cells were routinely cultured in a humidified atmosphere of 95 $\%$ air and $5 \% \mathrm{CO}_{2}$ at $37{ }^{\circ} \mathrm{C}$. The Caco2 cells were grown in $175 \mathrm{~cm}^{2}$ flask to form a confluent monolayer for 2 days. Afterwards, the cells were split by removing the old media, washing the monolayer with $5 \mathrm{ml}$ EDTA and detaching the cells with 5 $\mathrm{ml}$ of trypsin. Then $10 \mathrm{ml}$ of DMEM medium (10\% FCS and 1x non-essential amino acids) were added, the cells were centrifugated at $1380 \mathrm{rpm}$ for $5 \mathrm{~min}$. and recultivated in $175 \mathrm{~cm}^{2}$ cell culture flasks (10\% FCS, $1 \%$ NEAA, $1 \%$ Penicillin/streptomycin).

\subsubsection{Invasion and adhesion assays}

Bacterial invasion of host cells was initially described by Everest et al., (1992). After achieving a semiconfluent layer cells were washed with PBS and 400 $\mu \mathrm{l} C$. jejuni suspension in DMEM, (10 \% FCS and 1x NEAA) were added. To assure that the number of bacteria was identical in every assay performed, the solution was adjusted to an $\mathrm{OD}_{(600 \mathrm{~nm})}$ of 0.5 representing a multiplicity of infection (MOI) of 100 and incubated at $37{ }^{\circ} \mathrm{C}, 5 \% \mathrm{CO}_{2}$ to allow the bacteria to invade the host cell. In some experiments bacteria were brought in contact with the Caco2 cell by centrifugation on 6 well plates at $600 \times \mathrm{g}$ for 15 minutes. At $2 \mathrm{hrs}$ post infection, the cells were washed three times with 1x PBS before further incubation with culture medium containing $100 \mu \mathrm{g} / \mathrm{ml}$ gentamicin. After treatment with gentamicin for 2 hrs the cells were washed again three times with $1 x$ PBS. Then the cells were lysed with $1 \%$ Triton $\mathrm{X}-100$ for $10 \mathrm{~min}$ to release intracellular bacteria. Finally, the number of viable bacteria was determined by plating serial dilutions on Columbia blood agar and counting of the number of bacteria grown after incubation for $48 \mathrm{hrs}$ at $42{ }^{\circ} \mathrm{C}$ under microaerophilic conditions. Thereby, the number of colonies obtained after the invasion assay with wild-type strain B2, 
mutants and complemented mutants was defined as cfu in each experiment. In order to investigate bacterial adhesion, Caco2 cells were incubated with the bacteria for only $30 \mathrm{~min}$. After the monolayer was washed with PBS, the cells were lysed and the bacteria were plated on Columbia blood agar plates in order to determine the number of recovered bacteria as described above.

\subsubsection{Freezing and thawing of Caco2 cells}

The detached Caco2 cells were suspended in $40 \%$ DMEM and mixed with the same volume of 2x freezing solution (20\% DMSO, 40 \% FCS in DMEM). One mililiter aliquotes were kept for one day at $-80{ }^{\circ} \mathrm{C}$ and transferred into liquid nitrogen for long term storage. In order to thaw the frozen cells, a cryotube was taken from liquid nitrogen and incubated in a $37{ }^{\circ} \mathrm{C}$ water bath. After the cells were thawed they were cultured under the conditions described above.

\subsubsection{Modification of nucleic acids}

\subsubsection{Preparation of genomic DNA}

Genomic DNA of C. jejuni was isolated using the QIAamp DNA Mini Kit following the manufacturer's instructions. The bacteria were removed from culture plates and mixed with $180 \mu \mathrm{l}$ of Buffer ATL by vigorous shirring. Then $20 \mu \mathrm{l}$ of Proteinase $\mathrm{K}$ was added and the sample was incubated at $56{ }^{\circ} \mathrm{C}$ until the bacteria were completely lysed. After lysis $200 \mu \mathrm{l}$ of AL buffer was added and the sample was incubated at $70{ }^{0} \mathrm{C}$ for $10 \mathrm{~min}$. After mixing $200 \mu \mathrm{l}$ of ethanol (100\%) to the sample the lysate was transferred to a QIAamp spin column and centrifuged at $6,000 \mathrm{~g}$ for 1 minute. The column was washed once with $500 \mu \mathrm{l}$ of buffer AW1 and then with $500 \mu \mathrm{l}$ buffer AW2. Finally, the QIAamp spin column was transferred in a clean $1.5 \mathrm{ml}$ tube and the genomic DNA was eluted with $200 \mu \mathrm{l}$ Buffer AE.

\subsubsection{Isolation of plasmid DNA}

Plasmid DNA was isolated from $E$. coli cells using a plasmid purification kit (Sigma-Aldrich) according to the manufacturer's instructions. Bacteria from a $2 \mathrm{ml}$ 
of overnight culture were centrifugated and resuspented in $200 \mu \mathrm{l}$ resuspension solution. Then $350 \mu \mathrm{l}$ of neutralization solution was added and the sample was transferred to a spin column and centrifugated at maximum speed for 1 min. After washing the column with $750 \mu$ washing solution, the plasmid DNA eluted with 10 $\mu$ of the appropriate buffer.

\subsubsection{Isolation of RNA}

Bacterial RNA was extracted from C. jejuni cells with the RiboPure Bacter Kit (Ambion) following the recommendations of the protocol. After the over night culture was centrifugated, $350 \mu \mathrm{l}$ RNAwiz solution was added and the sample was transferred to a tube containing $250 \mu \mathrm{l}$ Zirconioa beads. After mixing for $10 \mathrm{~min}$ at maximum speed the sample was centrifugated for $5 \mathrm{~min}$ at $4{ }^{\circ} \mathrm{C}$. The supernatant was transferred to a fresh $1.5 \mathrm{ml}$ tube and mixed with $0.2 \times$ volume of chloroform. Then the sample was centrifugated for $5 \mathrm{~min}$ at $4{ }^{\circ} \mathrm{C}$ and the aqueous phase was conveyed to a fresh $1.5 \mathrm{ml}$ tube where $0.5 \mathrm{x}$ volume of $100 \%$ ethanol was added. The mixture was converted into filter cartilage for centrifugation at $13,000 \mathrm{rpm}$ for $1 \mathrm{~min}$. Afterwards the flow-through was discarded and the column was washed once with $700 \mu \mathrm{l}$ wash solution 1 and twice with wash solution 2/3. Finally, the RNA was eluted by applying $30 \mu$ of preheated $\left(95^{\circ} \mathrm{C}\right.$ to $\left.99{ }^{\circ} \mathrm{C}\right)$ elution solution to the centre of filter and centrifugation for $1 \mathrm{~min}$. After RNA isolation, DNA contaminations were removed by DNase I treatment and incubation at $37{ }^{\circ} \mathrm{C}$ for $30 \mathrm{~min}$ followed by denaturation of the enzyme by for $15 \mathrm{~min}$ at $75{ }^{\circ} \mathrm{C}$. To make sure that the RNA was free of remaining traces of DNA after DNase I treatment, PCR assays were performed after every preparation.

\subsubsection{Quantification of DNA and RNA}

Concentrations of DNA and RNA were determined with a NanoDrop ND 1000 spectrophotometer according to the instruction of the manufacturer. 


\subsubsection{Polymerase chain reaction (PCR)}

PCR was used to amplify $C$. jejuni genes for subsequent cloning into vectors pRRC or pBluescript II. The genomic DNA of wild type B2 and mutant were used as template to amplify the desired sequences, using PCR primers with or without introduced endonuclease restriction sites which are summarized in Table 2.2. Each $50 \mu \mathrm{l}$ of PCR mixture contained $40 \mathrm{ng}$ genomic DNA, $10 \mathrm{mM}$ TRIS-HCl pH 8.3, $50 \mathrm{mM} \mathrm{KCl}, 1.5 \mathrm{mM} \mathrm{MgCl}_{2}$, all four dNTPs (each $0.2 \mathrm{mM}$ ) and $2.5 \mathrm{U}$ Taq DNA polymerase. After initial incubation at $95{ }^{\circ} \mathrm{C}$ for $1 \mathrm{~min}, 40$ cycles at $95{ }^{\circ} \mathrm{C}$ for $30 \mathrm{~s}$, $55{ }^{\circ} \mathrm{C}$ for $30 \mathrm{~s}$ and $72{ }^{\circ} \mathrm{C}$ for $1 \mathrm{~min}$ were carried out with a final incubation at $72{ }^{\circ} \mathrm{C}$ for $10 \mathrm{~min}$. Different annealing temperatures were used based on the melting point of the respective primer pairs, while the length of the amplified region determined the elongation time (30 $\mathrm{s}$ for each $1 \mathrm{~kb}$ ). All PCR products were analysed on 1-1.5\% agarose gels stained with ethedium bromide at a final concentration of $1 \mu \mathrm{g} / \mathrm{ml}$. The size of PCR products was determined by using the $1 \mathrm{~kb}$ or $100 \mathrm{bp}$ GeneRulers DNA ladder which was run along with the PCR sample.

\subsubsection{Conventional reverse transcriptase-PCR}

Conventional (RT)-PCR assays were carried out with the OneStep RT-PCR Kit following the manufacturer's recommendations. After reverse transcription of 10 ng for $30 \mathrm{~min}$ at $50{ }^{\circ} \mathrm{C}, 35$ cycles of amplification were performed according to the following protocol: cDNA was denatured at $94{ }^{\circ} \mathrm{C}$ for $30 \mathrm{~s}$, primer annealed at 55 ${ }^{0} \mathrm{C}$ for $30 \mathrm{~s}$ and extended at $72{ }^{\circ} \mathrm{C}$ for $1 \mathrm{~min}$, with a final incubation at $72{ }^{\circ} \mathrm{C}$ for 10 min. The primers used for reverse transcriptase-PCR are listed in Table 2.3.

\subsubsection{Real-Time reverse transcriptase-PCR analysis}

Semi-quantitative real-time RT-PCR analysis was carried out with the LightCycler 1.5 and the QuantiFast SYBR Green RT-PCR Kit. LightCycler PCR assays were performed in glass capillaries in triplicate in a final volume of $20 \mu \mathrm{l}$ with $10 \mathrm{pmol}$ of each HPLC purified primer, $10 \mu \mathrm{l}$ of master mix and $50 \mathrm{ng}$. Amplicons of the constitutively transcribed $23 S$ gene were applied to adjust the RNA samples under 
investigation based on the crossing points obtained for this gene. 23S RT-PCR was carried out according to this protocol: initial reverse transcription for $20 \mathrm{~min}$ at $50{ }^{\circ} \mathrm{C}$ and denaturation for $5 \mathrm{~min}$ at $95{ }^{\circ} \mathrm{C}$ was followed by 25 cycles of denaturation (95 $\left.{ }^{\circ} \mathrm{C} ; 10 \mathrm{~s}\right)$, annealing $\left(50{ }^{\circ} \mathrm{C} ; 10 \mathrm{~s}\right)$ and elongation $\left(72{ }^{\circ} \mathrm{C} ; 5 \mathrm{~s}\right)$. After adjustment of the RNA samples real time RT-PCR assays for the genes under investigation were run. Therefore, reverse transcription was followed by 25 cycles of denaturation $\left(95{ }^{\circ} \mathrm{C}, 10 \mathrm{~s}\right)$, annealing $\left(55^{\circ} \mathrm{C} ; 10 \mathrm{~s}\right)$ and elongation $(72$ $\left.{ }^{0} \mathrm{C} ; 10 \mathrm{~s}\right)$. Each $\mathrm{PCR}$ included a negative control comprising of all elements without RNA to monitor possible contamination. The primers used for both assays are listed in Table 2.3. Every assay was run in duplicate. The specifity of the signal was assured by melting curve analysis and agarose gel electrophoresis. Semiquantification of transcription levels were calculated as follows: $x=2^{\Delta C p}$, where $x$ represents the factor of altered transcription, and $\Delta C p$ illustrates the difference of crossing points (Cp1-Cp2) of the two samples to be compared.

\subsubsection{Enzymatic digestion of DNA}

Purified PCR products harbouring the required endonuclease restriction sites and cloning vectors were digested by $10 \mathrm{U}$ restriction endonuclease in such a way that $10 \mu \mathrm{l}$ of purified PCR product or $1 \mu \mathrm{g}$ of cloning vector were incubated with $1 \mathrm{x}$ NEB buffer, $1 \times 10 \%$ BSA (optional) and $\mathrm{dH}_{2} \mathrm{O}$ in a volume of $20 \mu \mathrm{l}$ at $37{ }^{\circ} \mathrm{C}$ for 2 hrs.

\subsubsection{Purification of PCR products}

DNA extraction from agarose gels were performed using QIAGEN PCR Purification Kit according to manufacturer's protocol. DNA fragment was excised with a scalpel from the agarose gel, weighted and three volume of buffer QG to one volume of excised gel was added, respectively. The mixture was completely dissolved by incubating at $50{ }^{\circ} \mathrm{C}$ for $10 \mathrm{~min}$ and mixed thoroughly with $1 \mathrm{gel}$ volume of isoproponal. Then the sample was transferred to a QIAquick spin column placed in $2 \mathrm{ml}$ collection tube, centrifugated 13,000 rpm for one min and washed with $750 \mu \mathrm{l}$ of buffer PE. After all, the DNA was eluted by adding $30 \mu \mathrm{l}$ of 
elution buffer in the middle of column and final centrifugation at $8,000 \mathrm{rpm}$ for 1 $\min$.

\subsubsection{Ligation}

The restricted DNA fragments were ligated to a vector in a final volume $20 \mu \mathrm{l}$ containing 1:3 molar ratios of vector and insert, $1 \mu \mathrm{l}$ of Quick T4 DNA ligase, 1x ligation buffer and $d \mathrm{dH}_{2} \mathrm{O}$. The ligation reaction mixture was kept at room temperature for 1 hour or incubated over night at $16{ }^{\circ} \mathrm{C}$.

\subsubsection{Direct sequencing of genomic DNA from $C$. jejuni}

Each $10 \mu \mathrm{g}$ of genomic DNA were sequenced directly by the SeqLab Company (Göttingen, Germany) applying 10 pmol of KanF primer which is listed in Table 2.1.

\subsubsection{Experiments related to mutants of $C$. jejuni and their characterization}

\subsubsection{Knock-out inactivation of $c j 0005 c$}

Primers Cj0005cF and Cj0005cR (Table 2.2) were used to amplify a 1239 bp DNA fragment corresponding to $C$. jejuni gene cj0005c. The PCR fragment was Xbaldigested and inserted into plasmid vector pBluescript II KS to obtain pBcj0005c. To insert a kanamycin resistance cassette containing the aphA-3 gene, PCR was carried out with plasmid pSB1699 as a template and the 5'-phosphorylated primers Kan1 and Kan2 (Table 2.2). The PCR reaction contained $10 \mathrm{mM}$ Tris-HCl pH8.3, $50 \mathrm{mM} \mathrm{KCl}, 1.5 \mathrm{mM} \mathrm{MgCl}_{2}$, all four dNTPs (each $0.2 \mathrm{mM}$ ), 10 pmol of primers and $1 \mathrm{U}$ Pfu Polymerase to generate a blunt-end PCR product. After initial incubation at $95{ }^{\circ} \mathrm{C}$ for $1 \mathrm{~min}, 30$ cycles at $95{ }^{\circ} \mathrm{C}$ for $30 \mathrm{~s}, 55{ }^{\circ} \mathrm{C}$ for $30 \mathrm{~s}$ and $72{ }^{\circ} \mathrm{C}$ for $1 \mathrm{~min}$ followed, with a final incubation at $72{ }^{\circ} \mathrm{C}$ for $10 \mathrm{~min}$. Following purification of the PCR product using the QIAquick PCR Purification Kit (Qiagen), the kanamycin resistance cassette was cloned into plasmid pBcj0005c, which was BsaB1 blunt end restricted and dephosphorylated with Antarctic Phosphatase (New England Biolabs) to obtain pBcj0005cKan. 


\subsubsection{Cloning of $C$. jejuni genes into expression vector pRRC}

The $C$. jejuni genes were PCR-amplified and subsequently cloned into expression vector pRRC using primers harboring restriction sites for Xbal, respectively. The primer sequences with Xbal restriction sites are mentioned in Table 2.2. The Campylobacter expression vector pRRC contains a $\mathrm{Cam}^{r}$ gene cassette which is bounded by a $16 \mathrm{~S}$ sequence and tRNAs for alanine and isoleucine, respectively. Immediately downstream of the $\mathrm{Cam}^{r}$ gene cassette resides a single Xbal site to allow the expression of Xbal-cloned genes under the control of the constitutively expressed $\mathrm{Cam}^{r}$ gene promoter. After transformation of $\mathrm{C}$. jejuni with the recombinant plasmid, the $\mathrm{Cam}^{r}$ gene cassette together with the Xbal-cloned gene is integrated into one of the $16 \mathrm{~S}$ loci of the recipient cell via highly efficient double recombination (Karlyshev and Wren, 2005).

\subsubsection{Motility assay}

One microliter of an overnight culture of $C$. jejuni adjusted to an $\mathrm{OD}_{(600 \mathrm{~nm})}$ of 0.025 was sticked in the center of a $0.4 \%$ Mueller-Hinton agar plate with the help of a suited normalized inoculation loop. Afterwards, the plates were incubated at $42{ }^{\circ} \mathrm{C}$ under microaerophilic conditions for 36 hrs. The low concentration of the agar allows the bacteria to swarm, and to form a visible halo within the agar. Motility of the bacteria under investigation was judged by the expansion of the halos which was examined by the measurement of the respective radii. Each experiment was performed seven times.

\subsubsection{Chemotaxis assay}

For chemotaxis assays, bacteria were grown on Columbia blood agar plates overnight at $42{ }^{\circ} \mathrm{C}$ under microaerophilic conditions. Then, the bacteria were suspended in PBS ( $\mathrm{pH} 7.0$ ), adjusted spectrophotometrically to an $\mathrm{OD}_{(600 \mathrm{~nm})}$ of 1 and mixed (1:1) with temperate soft agar (0.8\%, BD, Heidelberg, Germany). Afterwards, $12 \mathrm{ml}$ of the bacterial soft agar suspension were poured into a petri dish and $6 \mathrm{~mm}$ discs sodden with $20 \mu \mathrm{l}$ of the respective test chemicals $(0.1 \mathrm{M}$ and $\mathrm{pH}$ 7.0) were placed on the solidified agar. Zones of bacterial attraction or 
repulsion were measured after four hours of incubation at $42{ }^{0} \mathrm{C}$ under microaerophilic conditions. The chemotaxis assays have been carried out four times, respectively.

\subsubsection{Autoagglutination assay}

Autoagglutination assays were performed as described by Misawa and Blaser (2000). Bacteria inoculation from petri dishes were suspended in PBS and adjusted to an $\mathrm{OD}_{(600)}$ of approximately 1.0. Bacterial suspensions of $2 \mathrm{ml}$ were incubated under a microaerophilic condition for $24 \mathrm{hrs}$ at $37{ }^{\circ} \mathrm{C}$. Then, $1 \mathrm{ml}$ of the suspensions were removed carefully and the $\mathrm{OD}_{(600)}$ was measured. Bacterial strains under investigation were measured five times. The obtain data were normalized following the protocol of Howards and co-workers (2009).

\subsubsection{Analysis of Protein Expression}

\subsubsection{Measurement of protein concentration}

Protein concentrations were quantified by BCA protein assay kit (Pierce) according to the manufactures instructions.

\subsubsection{Sodium dodecyl sulphate polyarcylamide gel electrophoresis (SDS-PAGE)}

Proteins were separated by SDS-PAGE. The acrylamide gel cast was set up by laying two spacer sandwiched between rectangular glass plates. Firstly, the separating gel (10\% or $12 \%$ ) was applied to the space. After the polymerization of the separating gel, the stocking gel $(4.4 \%)$ was pipetted on the top of the separating gel and a comb was inserted into the sandwiched plates. After the gel was solidified, the comb was removed and the gel was ready to use.

\subsubsection{Protein analysis by SDS-PAGE}

The bacteria were suspended in $8 \mathrm{M}$ urea and boiled for $10 \mathrm{~min}$ at $95{ }^{\circ} \mathrm{C}$ in order to lyse the cells. Equal amounts of cell lysates were mixed with $2 \times$ SDS-PAGE 
sample buffer and heated at $95{ }^{\circ} \mathrm{C}$ for $10 \mathrm{~min}$ before gelelectrophoretic separation. The SDS gel was run at $32 \mathrm{mV}$ for approximately $2 \mathrm{hrs}$. After electropheresis, gel was stained with Coomassie staining solution for 1 hour. Unspecific staining was removed by incubating the gel in $30 \%$ methanol and 10 $\%$ acetic acid. Finally, the Coomassie stained gel was sealed in cellophane and dried overnight.

\subsubsection{Purification of a $6 x$ His-tagged Protein from $C$. jejuni under denaturing conditions}

All steps were carried out according to the QIA expressionist protocol (Qiagen) with the solutions recommended for purification of $6 x$ His-tagged proteins under denaturating conditions. The cell were suspended in lysis buffer (100 mM $\mathrm{NaH}_{2} \mathrm{PO}_{4}, 8 \mathrm{M}$ urea, $10 \mathrm{mM}$ Tris- $\mathrm{HCl}, \mathrm{pH} 8.0$ ) and stirred on a shaker for $60 \mathrm{~min}$ at room temperature. After centrifugation at $10,000 \mathrm{~g}$ the supernatant was mixed with Ni-NTA beads ( $1 \mathrm{ml} \mathrm{Ni-NTA}$ slurry to $4 \mathrm{ml}$ cleared lysate) and gently mixed at $200 \mathrm{rpm}$ on a rotary shaker for $90 \mathrm{~min}$ at room temperature. Then the lysate $\mathrm{Ni}$ NTA was loaded carefully into an empty column and the flow through was collected. The column was washed twice with $4 \mathrm{ml}$ buffer $\mathrm{C}\left(100 \mathrm{mM} \mathrm{NaH} \mathrm{PO}_{4}\right.$, $10 \mathrm{mM}$ Tris- $\mathrm{HCl}, 8 \mathrm{M}$ urea, $\mathrm{pH}$ 6.3) and the his-tagged protein was eluted with elution buffers $\mathrm{D}\left(100 \mathrm{mM} \mathrm{NaH}_{2} \mathrm{PO}_{4}, 10 \mathrm{mM}\right.$ Tris- $\mathrm{HCl}, 8 \mathrm{M}$ urea, $\mathrm{pH}$ 5.9) and $\mathrm{E}$ (100 mM NaH${ }_{2} \mathrm{PO}_{4}, 10 \mathrm{mM}$ Tris- $\mathrm{HCl}, 8 \mathrm{M}$ urea, $\mathrm{pH} 4.5$ ) following the instruction of the manufacturer.

\subsubsection{Western blotting}

Proteins separated on $12 \%$ SDS-PAGE were transferred to a PVDF membrane (GE Healthcare) using a semidry transport system (Sartorius). For this the blot sandwich was build up in the following arrangement. Anode (+): soaked 6 Whatman filter papers in $0.3 \mathrm{M}$ Tris- $\mathrm{HCl}, \mathrm{pH} 10.4$ and $20 \%$ methanol, soaked 3 Whatman filter papers in $25 \mathrm{mM}$ Tris- $\mathrm{HCl}, \mathrm{pH} 10.4$ and $20 \%$ methanol, nitrocellulose membrane gel, 9 Whatman filter papers soaked in $40 \mathrm{mM} \mathrm{6-}$ aminocapronic acid, $\mathrm{pH} 7.6$ and $20 \%$ methanol, Cathode (-) . The proteins were 
transferred at $0.9 \mathrm{~mA} / \mathrm{cm}^{2}$ of gel size for $90 \mathrm{~min}$. The membrane was blocked for $1 \mathrm{~h}$ with $5 \%$ milk powder in PBS containing $0.05 \%$ Tween 20. After incubation of the membrane with a 1:3,000 diluted monoclonal mouse anti His primary antibody over night at $4{ }^{\circ} \mathrm{C}$, the immune complexes were labelled with a 1:3000 diluted horseradish-peroxidase-conjugated anti mouse secondary antibody for $1 \mathrm{~h}$ at room temperature. The membrane was washed $4 \times$ for 20 min with $0.05 \%$ Tween 20 and incubated with ECL reagent (GE Health Care). Finally the membrane was covered by plastic foil and visualized by ECL chemoluminiscence following the manufacturer's recommendations.

\subsubsection{Statistical analysis}

Significant differences between mean values were calculated by the Student's $t$ Test using MS-Excel software. $P$-values of less than 0.01 were considered to be significant. 


\section{RESULTS}

\subsection{Invasion capacity of $C$. jejuni isolate B2 and the $C$. jejuni strains NCTC 11168 and 81-176}

Invasion experiments using Caco2 cells were performed in order to determine the infectivity of $C$. jejuni strain B2 compared to the well characterized $C$. jejuni strains NCTC 11168 and 81-176 by performing gentamicin protection assays. Thereby, it was observed that isolate B2 is more invasive than both reference strains. The result of this experiment is shown in Fig. 3.1. The mean value of colonies that were recovered from infections with the clinical isolate B2 was $1.2 \times 10^{6} \mathrm{cfu} / \mathrm{ml}$, whereas $0.85 \times 10^{5}$ and $4.1 \times 10^{5} \mathrm{cfu} / \mathrm{ml}$ were counted for, NCTC 11168 and 81-176, respectively.

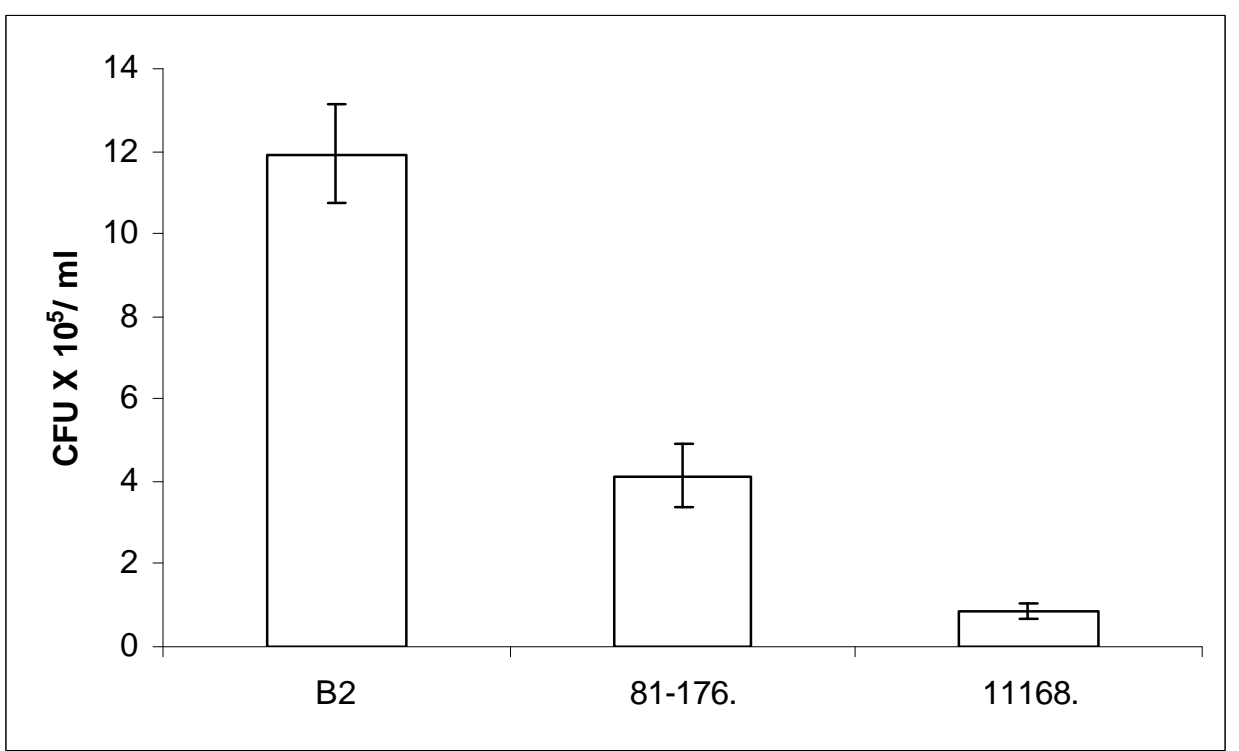

Figure. 3.1 Comparative analysis of the $C$. jejuni strains B2, NCTC 11168 and 81-176

Invasion assays using Caco2 cells showed increased infectivity of clinical isolate B2. The mean values of colonies recovered from NCTC 11168 and $81-176$ were $0.85 \times 10^{5}$ and $4.1 \times 10^{5} \mathrm{cfu} / \mathrm{ml}$. Compared to these two reference strains, the infectivity of the clinical isolate B2 was clearly higher with $1.2 \times 10^{6} \mathrm{cfu} / \mathrm{ml}$. 


\subsection{Identification of invasion related $\boldsymbol{C}$. jejuni genes}

To identify candidate genes that mediate a less invasive activity of $C$. jejuni to invade Caco2 cells, altogether 660 clones of an existing transposon-based mutant library of the C. jejuni strain B2 which was kindly provided by Dr. J. I. Dasti, (University of Gottingen) were individually analysed by performing gentamicin protection assays. Out of these 660 clones, seven clones have been detected with a strongly decreased invasiveness (Fig 3.2). Direct genomic sequencing was applied in order to determine the transposon insertion site within the genome of the respective $C$. jejuni clones, using a primer that binds directly to the 5 ' region of the kanamycin resistance cassette and, therefore, allows the determination of the affected $C$. jejuni genes. In order to approve the reduced invasiveness, gentamicin protection assays with the respective clones and wild-type strain B2 were repeated five times. Thereby, the mean value of colonies recovered from clinical isolate B2 were $1.1 \times 10^{6} \mathrm{cfu} / \mathrm{ml}$ whereas the $\mathrm{cfu} / \mathrm{ml}$ obtained for the respective mutants were as follows: cj0005c: $2,8 \times 10^{5} \mathrm{cfu} / \mathrm{ml}$, cj0078c: $3.4 \times 10^{5}$ cfu/ml, cj0093: $4.1 \times 10^{5} \mathrm{cfu} / \mathrm{ml}$, cj0268c: $3.3 \times 10^{5} \mathrm{cfu} / \mathrm{ml}$, cj0721c: $3.2 \times 10^{5} \mathrm{cfu} / \mathrm{ml}$, cj1439c: $3 \times 10^{5} \mathrm{cfu} / \mathrm{ml}$, and cj0952c: $2.3 \times 10^{5} \mathrm{cfu} / \mathrm{ml}$ with P-values of $<0.001$, respectively. The results are summarized in Fig. 3.2 and table 3.1. 


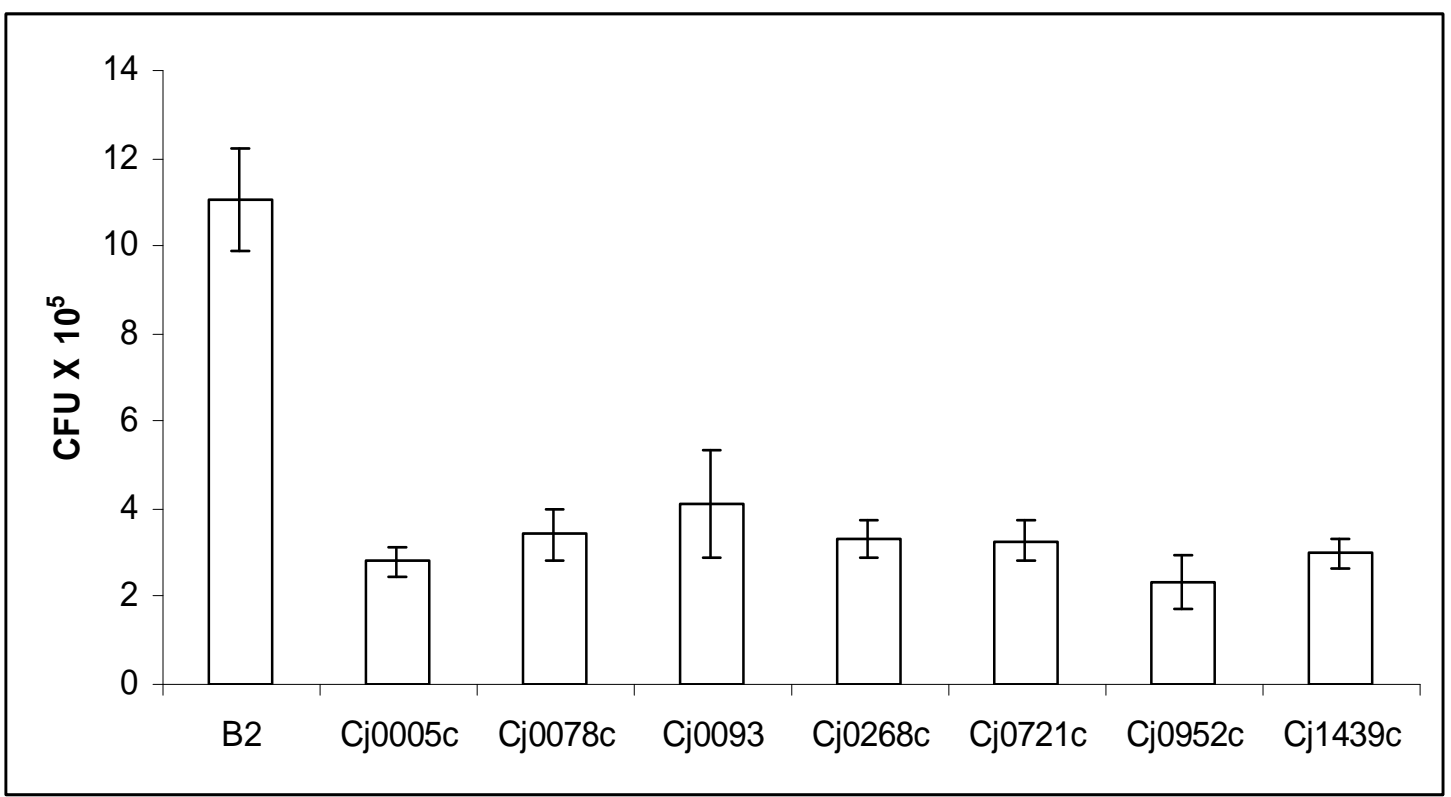

Figure 3.2 Invasion of the B2 wild-type strain and selected clones of a transposon based mutant library

The names of clones correspond to the mutated gene. See text for details.

Table 3.1 Specification of the detected genes with regard to function and localization as far as known

\begin{tabular}{|l|l|}
\hline Gene & Putative Function \\
\hline cj1439c & UPD-galactopyranose mutase \\
\hline cj0952c & N-terminal of a chemoreceptor \\
\hline cj0721c & Integral membrane protein \\
\hline cj0268c & Transmembrane protein \\
\hline cj0093 & Periplasmic protein \\
\hline cj0078c & CdtB, cytolethal distending toxin \\
\hline cj0005c & Sulphite:cytochrome c oxidoreductase \\
\hline
\end{tabular}


Gene cj0952c represents the N-terminus of a putative chemoreceptor, whereas cj0005c encoding one subunit of a sulphite: cytochrome c oxidoreductase (SOR). Since to my knowledge neither a chemoreceptor nor an enzyme from the energy metabolism has been connected to the infectivity of $C$. jejuni so far, these two genes were selected for further investigation.

\subsection{Characterization of $c j 0952 c$}

3.3.1 Transposon insertion in gene cj0952c does not alter the transcription of both upstream gene cj0953c as well as downstream gene cj0951c

To determine the effect of the transposone insertion into gene cj0952c on the transcription of the upstream and downstream genes, reverse transcription PCR assays with the wild-type strain B2 and the mutant B2 $\Delta c j 0952 c$. was performed The non-coding sequence between $c j 0953 c$ and $c j 0952 c$ has a length of $96 \mathrm{bp}$, and the gap between cj0952c and cj0951c is 263 bp in the clinical isolate B2, indicating a transcription of these three genes in a polycistronic fashion. For this, an altered transcription of especially the downstream located gene cj0951c cannot be excluded in case of a transposon insertion within gene cj0952c (Fig. 3.3). As it is shown in Fig. 3.4, transcription of cj0952c including the transposon, but also of cj0953c and cj0951c could clearly be proven in the mutant B2 $\Delta c j 0952 c$, ensuring the invasion-deficient phenotype to be exclusively mediated by the insertion of the transposon into cj0952c. 


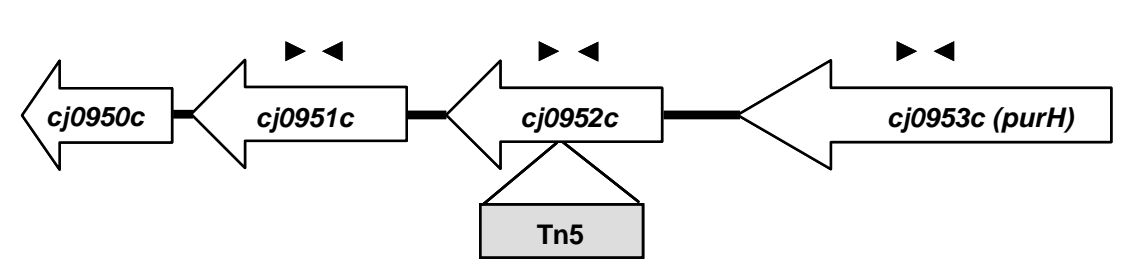

Figure. 3.3 Genomic composition of cj0953c, cj0952c, cj0951c and cj0950c in the clinical $C$. jejuni isolate B2

In the above vectorial diagram the directions of the arrows denotes the transcriptional orientation of the genes. Primers used for RT-PCR analysis are shown as arrowheads. The Insertion site of the transposon is indicated. (Genbank accession number GU799572).

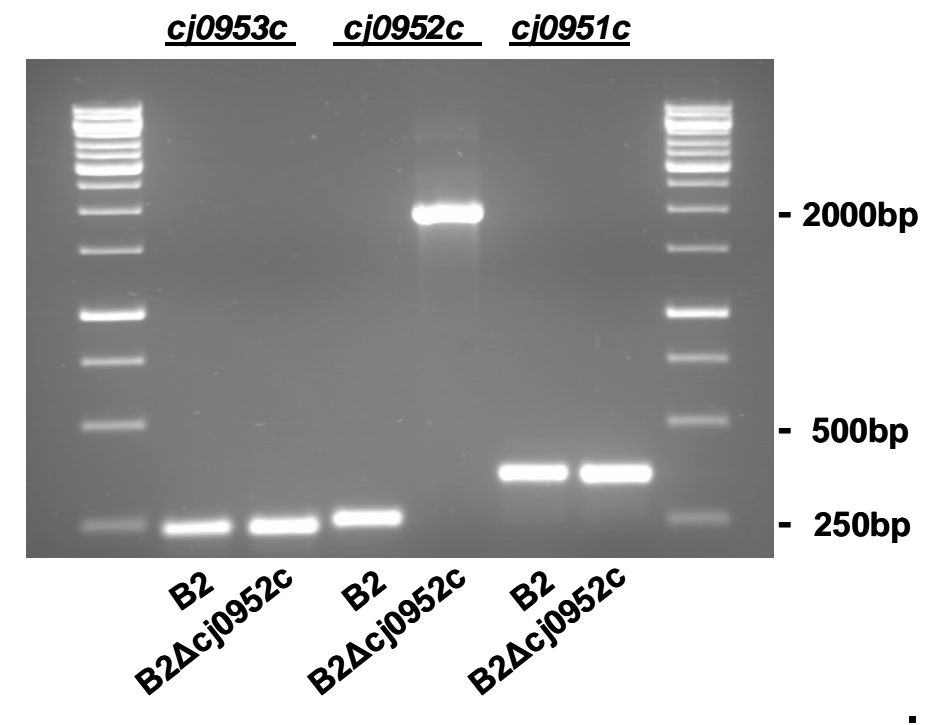

Figure 3.4 RT-PCR assays for the investigation of $C$. jejuni genes cj0951c, cj0952c and cj0953c in wild-type B2 strain and the mutant strain B2Acj0952c

The PCR amplicons are displaying the expected size for cj0953c (225 bp), cj0952c wild-type (267 bp), cj0952c with the integrated transposon (2091 bp) and cj0951c (356 bp). To confirm RNA purification, PCR analysis on RNA templates under investigation yielded no amplicons, reflecting that DNA contaminations were completely removed (not shown).

\subsubsection{Functional restoration of $C$. jejuni $\mathrm{B} 2 \Delta$ cj0952C}

To restore the infectivity of the B2Acj0952c mutant, cj0952c and cj0951c alone and cj0952c combined with cj0951c have been cloned into vector pRRC and were 
channelled into B2Acj0952c. Subsequent gentamicin protection assays on Caco2 cells restored the parental phenotype only in the presence of both genes. Neither cj0952c nor cj0951c alone increased the mutant infectivity towards the wild-type B2. A graphical representation of altogether five independent experiments is shown in Fig 3.5.

Unlike to wild-type strain B2, transcription of cj0952c in the complemented mutants is under control of a cam ${ }^{R}$ promoter. Real time RT PCR was performed in order to compare the transcription level of cj0952c in the wild-type and the complemented mutants. RNA samples adjustment was performed according to the crossing points of the $23 S$ transcripts, respectively. Then, the cj0952c transcription level of the wild type strain, the mutant B2 $\Delta c j 0952 c$ complemented with cj0952c alone and complemented with cj0952c-cj0951c were determined. While the average crossing points of both complemented mutants were 20.49 (cj0952c) and 20.18 (cj0952c-cj0951c), a specific signal for cj0952c from wild type strain was primal obtained at a crossing point of 32.44 , clearly demonstrating a more than 4000 fold upregulation of cj0952c in the complemented mutants compared to the wild type strain. Consequently, a low transcription level of cj0952c under the control of the $\mathrm{cam}^{R}$ promoter could be excluded as a reason for the incomplete invasion phenotype of the mutant which only habours the cj0952c gene.

In contrast to the clinical isolate B2 and C. jejuni strain NCTC 11168 where the putative chemoreceptor Cj0952c-Cj0951c is coated by two individual genes, the corresponding putative chemoreceptor in $C$. jejuni strain $81-176$ is encoded by a single gene that covers the transmembrane domain, the HAMP domain and also the MCP domain. To further investigate whether the single gene of $C$. jejuni 81176 is able to complement B2 $\Delta$ cj0952c, was cloned it into pRRC and introduced the recombinant plasmid into the mutant. Thereby, the Caco2 cell infection was also restored up to the wild type level as summarized in Fig. 3.5. 
In a next attempt to restore the invasiveness of the mutant B2 $\Delta$ cj0952c completely, a PCR fragment encompassing cj0952c-cj0951c-cj0950c was cloned into vector pRRC. Gene cj0950c is located downstream of cj0951c and the corresponding gene product is a putative lipoprotein of yet unknown function. It possesses a predicted signal peptide and shows homology to the heat shock protein HSLJ of $E$. coli. Since nothing is known about the role of this gene, a functional correlation of Cj0950c with Cj0952c and Cj0951c seemed to be possible. Gentamicin protection assays after introduction of the recombinant plasmid carrying all three genes obtained the recovery rates comparable to the mutant complemented with cj0952c-cj0951c indicating that Cj0950c is not in a functional correlation with Cj0952c and Cj0951c (Fig. 3.5 and Table 3.2).

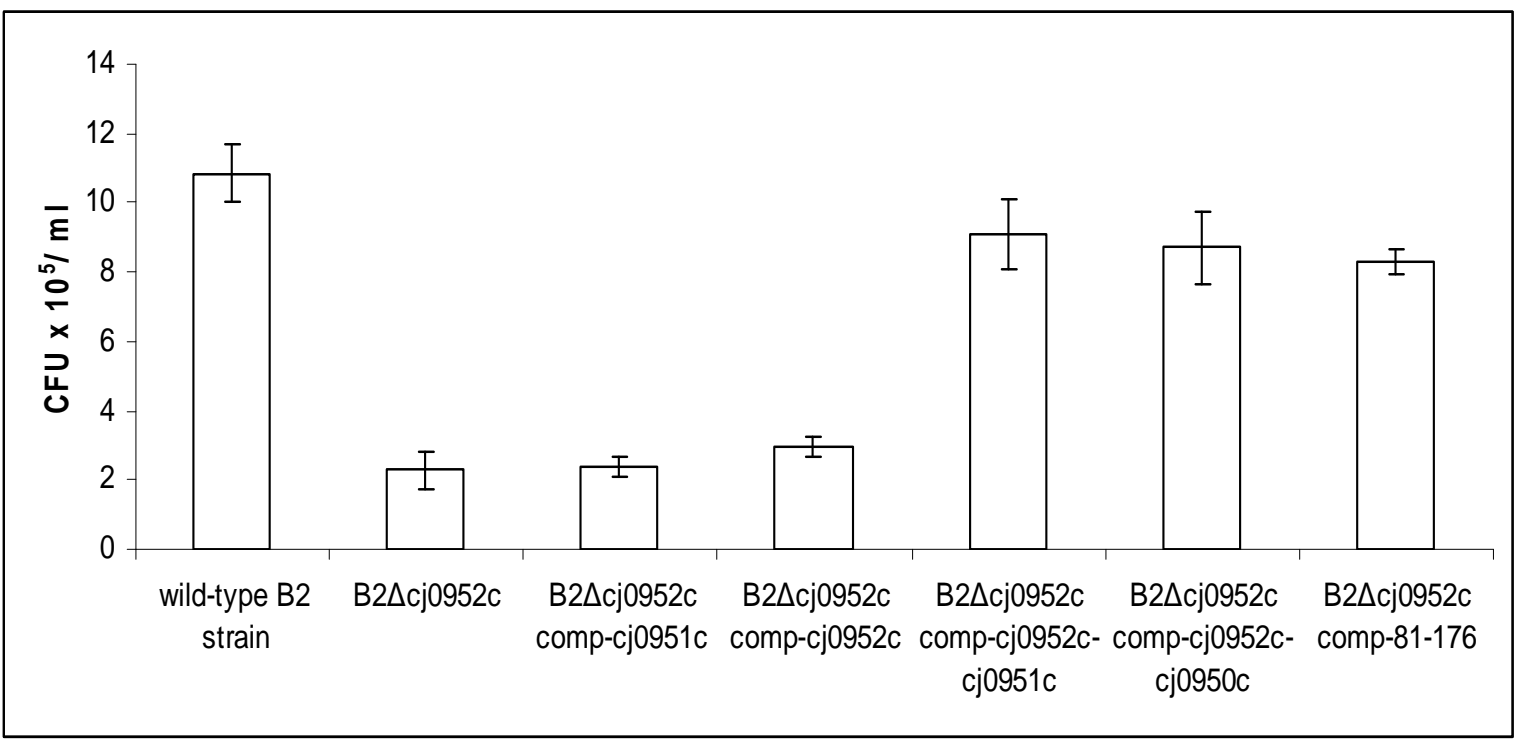

Figure 3.5 Infection of Caco2 cells by wild-type strain B2, the mutant and the complemented mutants

Investigation revealed that the reconstitution of $C$. jejuni wild-type B2 strain invasiveness was achieved by complementation of B2 $\Delta c j 0952 c$ with $c j 0952 c-c j 0951 c$ but not with $c j 0952 c$ or $c j 0951 c$ alone. The additional expression of Cj0950c had no influence on the recovery rate. Complementation of $\mathrm{B} 2 \Delta c j 0952 C$ with the corresponding gene of $C$. jejuni strain 81-176 (B2Acj0952c-comp-81-176) also shifted the infectivity of the mutant back to wild-type level. The experiments were carried out in triplicate and have been repeated five times. The error bars represent the standard deviation. 
Table 3.2 The mean values of colonies recovered after five independent invasion experiments

\begin{tabular}{lcc}
\hline Strain & Cfu/ml $\left(\times 10^{5}\right)$ & Standard deviation $\left(\times 10^{5}\right)$ \\
\hline wild-type B2 & 10.86 & \pm 0.86 \\
B2 $\Delta$ cj0952c & 2.30 & \pm 0.53 \\
B2 $\Delta$ cj0952c comp-cj0952c & 2.98 & \pm 0.28 \\
B2 $\Delta$ cj0952c comp-cj0951c & 2.38 & \pm 0.26 \\
B2 $\Delta$ cj0952c comp-cj0952c- & 9.08 & \pm 1.00 \\
cj0951c & & \\
B2 $\Delta$ cj0952c comp-cj0952c- & 8.72 & \pm 1.04 \\
cj0950c & & \\
B2 $\Delta$ cj0952c comp-81-176 & 8.30 & \pm 0.37 \\
\end{tabular}

The parental strain B2 was significantly more invasive as compared to the reduced invasiveness of the mutant B2Acj0952c and the mutants complemented with cj0952c or cj0951c $(\mathrm{P}<0.001)$. The variation of recovered colony numbers after invasion assays with mutants complemented with cj0952c-cj0951c, cj0952c-cj0950c or 81-176 was subsidiary ( $\mathrm{P}>0.001)$. The standard deviations are indicated. See text for details.

\subsubsection{C. jejuni genes cj0952c and cj0951c of the clinical isolate B2 are translated into separate proteins}

C. jejuni genes cj0951c and cj0952c in the clinical isolated B2 as well as in the strain NCTC 11168 have been annotated to be pseudogenes, postulated to be translated into one functional protein by a speculated read through mechanism. To prove this hypothesis, PCR and reverse transcription-PCR with DNA and RNA of the parental strain B2 was carried out to detect alterations of the synthesized RNA e.g. a stop codon replacement of cj0952c to create one open reading frame for both genes. PCR and reverse transcription-PCR assays have been carried out with the primers Cj0952c-51cF and Cj0952c-51cR (listed in Table 2.1) which are spanning the 3'-region of cj0952c, the intergenic region and the 5'-domain of cj0951c. After sequencing of the PCR and RT-PCR products no differences between both amplicons could be detected. Neither the stop codon of cj0952c at 
position 891663 of the genome was converted, nor other changes within the RNA sequence could be found contradicting the idea of a postulated read-through mechanism (data not shown). However, the analysis of the RT-PCR amplicon could justify that cj0951c and cj0952c are co-transcribed on one mRNA. Afterwards, genes cj0952c and cj0951c together were cloned into vector pRRC to answer the question whether these genes are translated as one protein or separately. For the proof of the recombinant protein expression, the primer corresponding to the 3'-end of cj0951c was extended by nucleotides representing a $6 x$ His-tag (Table 2.2). After introduction of the recombinant plasmid into mutant $\mathrm{B} 2 \Delta$ cj0952c, the proteome was analysed by Western blotting. As a negative control, lysate of parental strain B2 was used. Thereby, a band corresponding to approximately $25 \mathrm{kDa}$ was obtained, which is going along with the predicted protein size of Cj0951c but not with the expected size for Cj0952c-Cj0951c (58 $\mathrm{kDa}$ ), confirming that no read-through mechanism takes place in $C$. jejuni strain B2 (Fig. 3.6). For the confirmation of the immunoblot results, the experiment with other randomly chosen clones which harboured the recombinant plasmid was repeated. While in most of the clones expression of the corresponding protein could not be detected, the same $25 \mathrm{kDa}$ bands were observed in two other clones. No band was found for a recombinant protein with the size of approximately $58 \mathrm{kDa}$ (data not shown). 


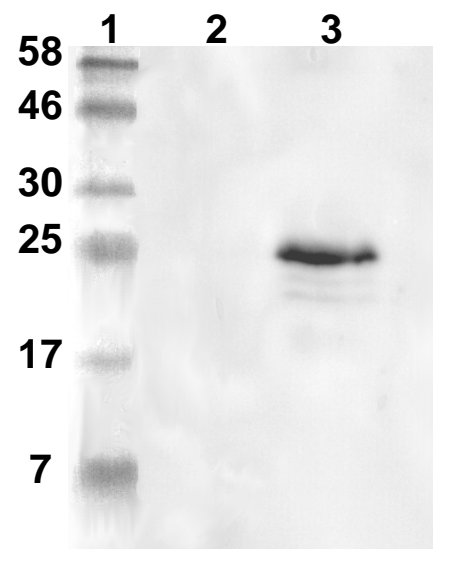

\section{Figure 3.6 Detection of the recombinant protein in $C$. jejuni by Immunobloting}

Lane 1: molecular mass marker (molecular masses are indicated in $\mathrm{kDa}$ ), lane 2: wild-type strain B2, lane 3: mutant B2Acj0952c-comp-cj0952c-cj0951cHis. The observed protein molecule exhibited a molecular mass of approximately $25 \mathrm{kDa}$, which resembled the size of Cj0951c. No protein of a molecular mass of $58 \mathrm{kDa}$ which would indicate a Cj0952c-Cj0951c composition could be detected.

\subsubsection{Decreased motility of $C$. jejuni B2 $\Delta$ cj0952c}

The chemotaxis machinery of a pathogen is known to be connected to motility (Hugdahl et al., 1988). For this, motility assays were used to investigate whether the mutant B2 $\Delta c j 0952 C$ has an altered phenotype. Thereby, a significantly reduced motility of the mutant compared to the parental strain B2 was determined. While the average diameter of the motility zone on semi-solid agar plates for B2 was $47.4 \mathrm{~mm} \pm 3.13$, the mutant was clearly less motile with a mean diameter of $30.6 \mathrm{~mm} \pm 2.4 \quad(\mathrm{P}<0.0001)$. After complementation of the mutant with cj0952c or cj0951c, the motility remained reduced compared to the parental strain $(30.6 \mathrm{~mm}$ $\pm 2.9,29.4 \mathrm{~mm} \pm 2.3, \mathrm{P}<0.0001)$. In contrast, when cj0952c together with cj0951c was introduced into the mutant, the motility of the wild-type was restored (46.6 $\mathrm{mm} \pm 3.2$. . Also the introduction of the corresponding gene of $C$. jejuni 81-176 into the mutant was successful to restore the parental phenotype. The motility zone of this revertant was $46.2 \mathrm{~mm} \pm 2.8$. The results are shown in Fig. 3.7 and 3.8. 

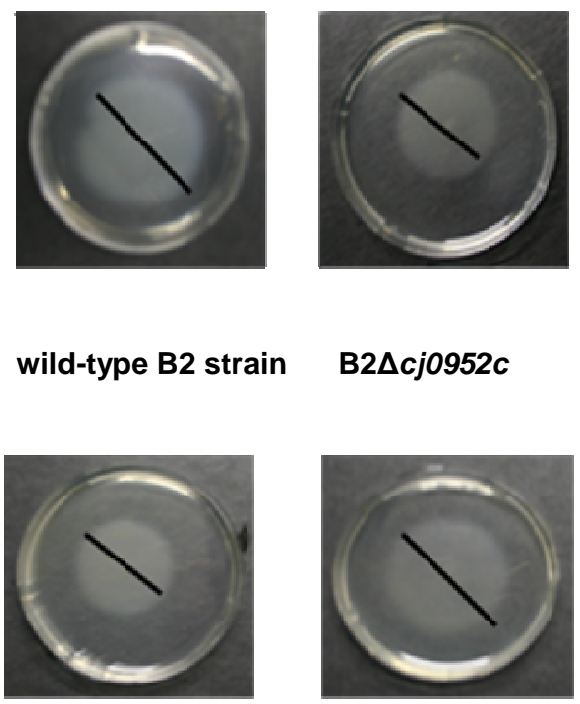

B2Acj0952c

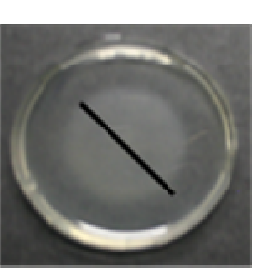

B2Acj0952c-

comp-cj0952c
B2 $\Delta$ cj0952c-compcj0952c-cj0951c

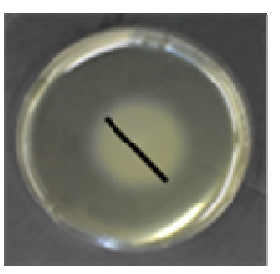

B2 $\Delta c j 0952 c-$

$-c j 0591 c$

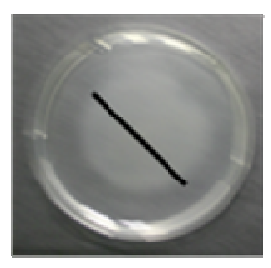

B2Acj0952c-

comp-81-176

Figure 3.7 Motility of wild type strain, complemented mutants and cj0952c-mutant of $C$. jejuni strain $\mathrm{B} 2$

The motility of B2 $\Delta c j 0952 c$ compared to wild-type strain B2 is not restored after introduction of cj0952c or cj0951c alone but only in combination of cj0952c and cj0951c or introduction of the corresponding gene from $C$. jejuni strain 81-176. Diagonal lines show the bacterial spread on the agar plates. 


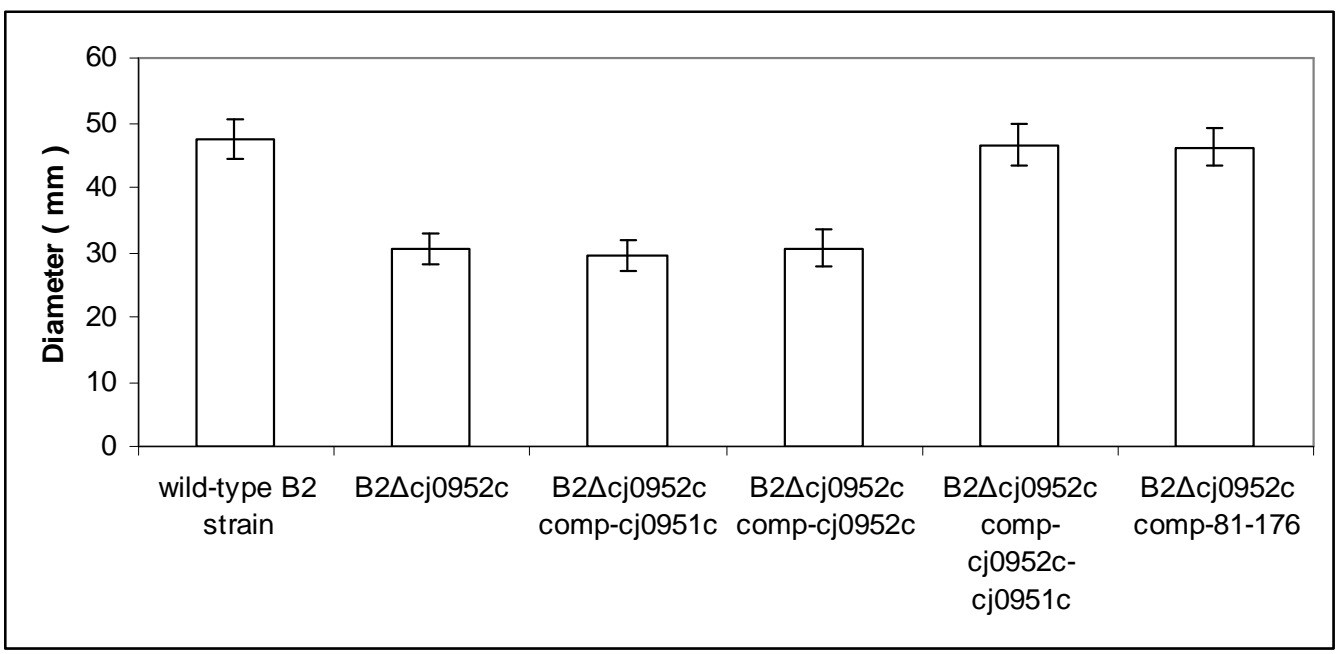

Figure 3.8 Motility zones of the wild-type strain B2, the mutant B2 $\Delta$ cj0952c and the complemented mutants

Motility assays were carried out on semisolid $0.4 \%$ Mueller Hinton agar plates inoculated with the indicated strains and incubated at $42{ }^{\circ} \mathrm{C}$ under microaerophilic conditions. Each experiment was performed seven times. See text for details.

\subsubsection{The C. jejuni proteins Cj0952c and Cj0951c alters the chemotactical behaviour of the pathogen in the presence of formic acid}

Chemotaxis assays were performed to investigate the chemotactical behaviour of the B2 $\Delta c j 0952 c$ mutant compared to that of the wild type strain B2 in order to investigate the function of Cj0952c-Cj0951c. For both strains, the accumulation of bacterial cells indicate chemoattraction around the plugs containing L-asparagine, L-aspartate, L-cysteine, fumarate, L-glutamate, D-lactate, L-(-)-malate, pyruvate, $\mathrm{L}$-serine and succinate. Repulsion was demonstrated around the plugs containing cholic acid, deoxycholic acid, glycocholic acid and taucholic acid while no taxis response could be detected from PBS, L-fucose, and citrate. No significant differences in the extent of attraction or repulsion between wild type strain B2 and the mutant B2 $\triangle c j 0952 c$ could be identified (not shown).

In contrast, in the case of formic acid a clear difference was observed. It was recently described that formic acid serves as chemoattractant for $C$. jejuni (Vegge 
et al., 2009). In accordance with the results of this publication, we demonstrated a significant attraction halo for the parental strain B2 around the fomate-sodden paper disc, whereas for B2 $\Delta$ cj0952c, a biphasic halo with an inner repulsion zone surrounded by a diminished ring was observed (Fig. 3.9). The chemotactical behaviour was restored by complementation of the mutant with cj0952c-cj0951c or the corresponding gene from $C$. jejuni 81-176, but no restoration occurred when the mutant was complemented with cj0952c or cj0951c alone (Fig 3.9). After four independent experiments, the attraction zones from B2 and the mutant complemented with genes cj0952c and cj0951c as well as the gene from $C$. jejuni 81-176 were $26.5 \mathrm{~mm}( \pm 2.6), 25 \mathrm{~mm}( \pm 2.5)$ and $25.5 \mathrm{~mm}( \pm 2.6)$, whereas the attraction zones of the mutant and the mutant complemented either with cj0952c or cj0951c alone were $10.2 \mathrm{~mm}( \pm 0.9), 10.0 \mathrm{~mm}( \pm 1.4)$ and $10.2 \mathrm{~mm}( \pm 1.7)$ as shown in Table 3.3. These results gave strong evidence that the genes cj0952c and cj0951c together are encoding a chemoreceptor which is involved in the chemotactical recognition of formic acid. 

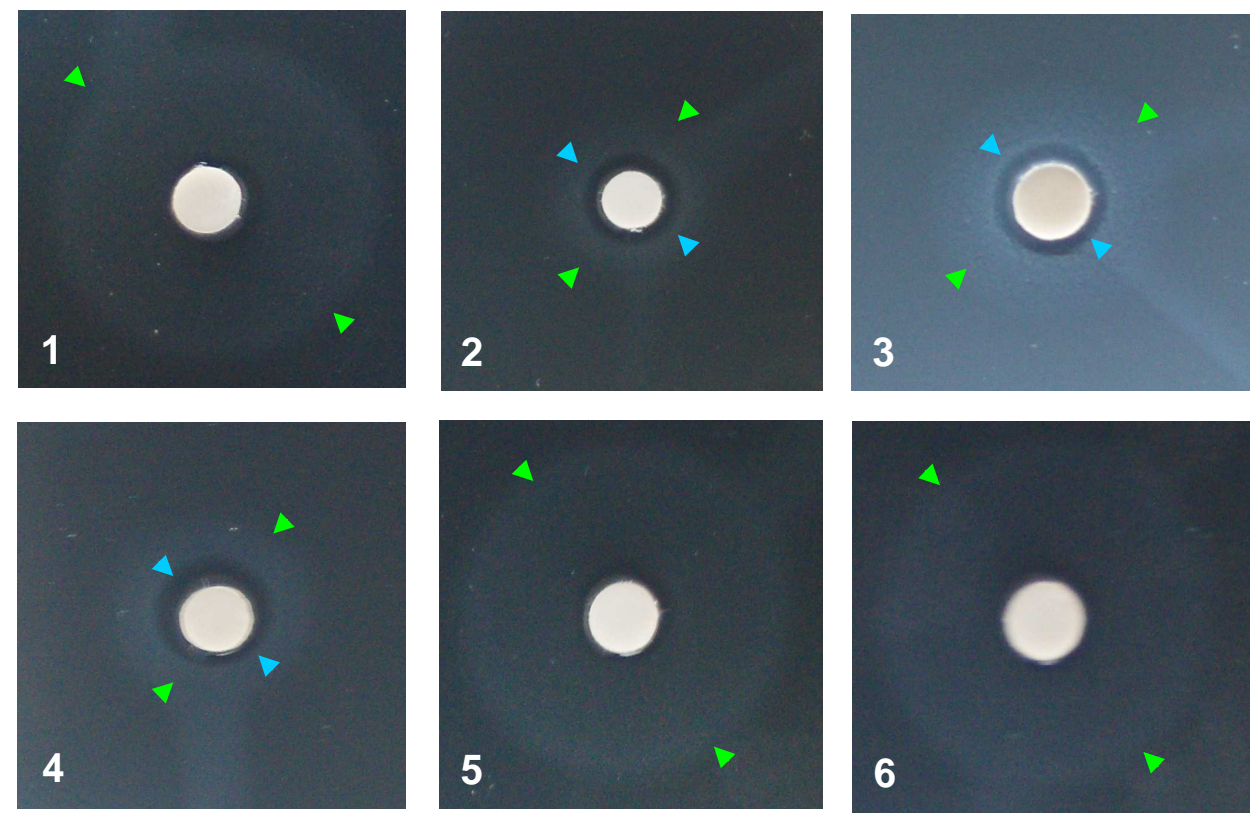

Figure 3.9 Photographical representation of the chemotactical behaviour of the investigated strains in the presence of formic acid

1. wild-type strain B2, 2. B2 $\Delta c j 0952 c$, 3. B2 $\Delta$ cj0952c-comp-cj0951c, 4. B2 $\Delta c j 0952 c$-comp-cj0952c 5. B2Acj0952c-comp-cj0952c-cj0951c, 6. B2 $\Delta c j 0952 c-c o m p-81-176$. Whereas wild-type strain B2, the mutant strain B2Acj0952c complemented with cj0952c-cj0951c or the corresponding gene from $C$. jejini strain 81-176 clearly demonstrated chemoattraction around the formate sodden paper (green arrows), mutant B2 $\triangle c j 0952 c$ as the mutant complemented with cj0952c or cj0951c alone demonstrated a diversified chemotactical response: an inner repulsion zone (blue arrow) could be observed surrounded by a dense chemo attractant ring (green arrow). 
Table 3.3 Chemoattraction zones of $C$. jejuni wild type strain, Complemented mutants and mutant are indicated in $\mathbf{~ m m}$

\begin{tabular}{llc}
\hline Strain & $\begin{array}{l}\text { Chemoattraction zone } \\
(\mathrm{mm})\end{array}$ & $\begin{array}{l}\text { Standard deviation } \\
(\mathrm{mm})\end{array}$ \\
\hline wild-type B2 & 26.50 & \pm 2.64 \\
B2 $\Delta$ cj0952c & 10.25 & \pm 0.95 \\
B2 $\Delta$ cj0952c comp-cj0951c & 10.25 & \pm 1.70 \\
B2 $\Delta$ cj0952c comp-cj0952c & 10.00 & \pm 1.41 \\
B2 $\Delta$ cj0952c comp-cj0952c- & 25.00 & \pm 2.58 \\
cj0951c & & \pm 2.64 \\
B2 $\Delta$ cj0952c comp-81-176 & 25.5 & \\
\end{tabular}

Chemotactical response of wild-type B2 and the mutants B2 $\Delta$ cj0952c complemented with cj0952ccj0951c or the respective gene from strain 81-176 are identical. In contrast, neither cj0952c nor cj0951c did restore the parental phenotype and exhibited significantly reduced chemotaxis $(P<0.001)$ towards formic acid.

\subsubsection{The presence or absence of Cj0952c-Cj0951c does not affect the usage of formic acid}

Both, immunoblot analysis and molecular genetic approaches indicate that both proteins are translated separately and not as a single chemoreceptor. For further investigation of this unusual composed chemoreceptor, Cj0952c-Cj0951c we examined if this composition is correlated with the utilization of formic acid. The growth of the wild-type strain B2, the mutant, the cj0952c-cj0951c-complemented mutant and the mutant harbouring the single gene from $C$. jejuni 81-176 was measured in the presence of $10 \mathrm{mM}$ formic acid. As a negative control, a formate dehydrogenase-deficient mutant of the B2 strain (B2 $f d h$ ) was used. Thereby, no correlation of the chemoreceptor presence or composition and formic acid utilization was found. Wild type strain B2, the cj0952c-mutant and the complemented mutants showed exponentially identical proliferation while the growth of B2 $\Delta f d h$ was clearly reduced (Fig 3.10a). Increased growth of bacterial 
strains under investigation in the absence of formic acid demonstrated comparable results although the growth of $\mathrm{B} 2 \Delta f d h$ was slightly shifted, eventually due to the presence of minor traces of formic acid in the $\mathrm{MH}$ broth which could not be metabolized by $\mathrm{B} 2 \Delta f d h$ (Fig 3.10b).

a)

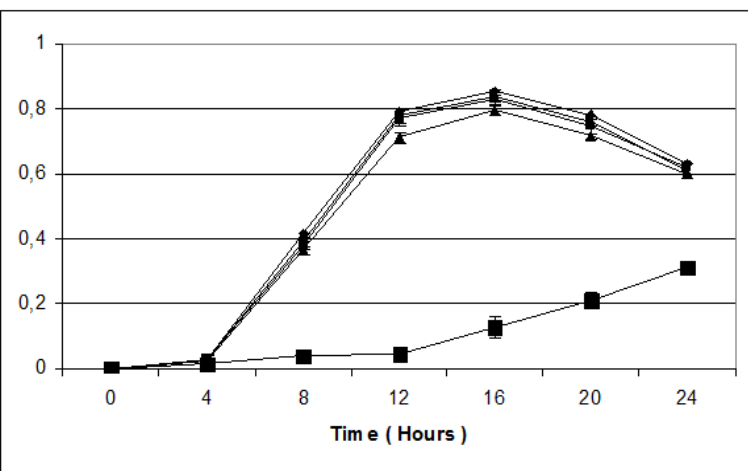

b)

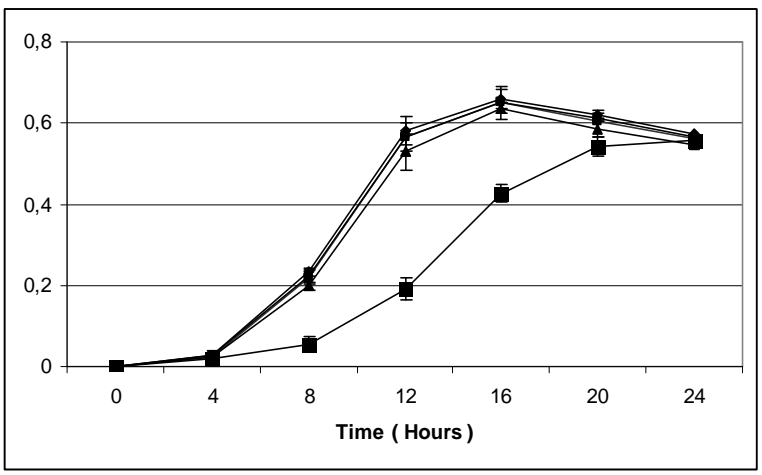

Figure 3.10 Growth curve with and without $10 \mathrm{mM}$ formic acid

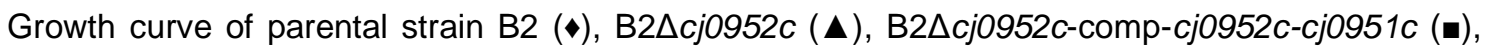

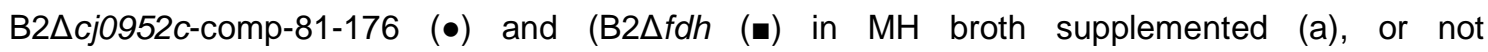
supplemented (b) with $10 \mathrm{mM}$ formic acid. The y axis shows the optical density measured at 600 $\mathrm{nm}$. Error bars are indicated. See text for details.

\subsection{Characterization of cj0005c}

The gene product of $c j 0005 c$ is encoding a molybdopterin oxidoreductase which, together with the monohaem cytochrome c oxidoreductase (cj0004c), constitutes a sulphite:cytochrome c oxidoreductase (SOR). Gentamicin protection assays on Caco2 cells with parental strain B2 and the mutant B2 $\Delta$ cj0005c clearly demonstrated the declined infectivity of the mutant compared to the wild type strain (Fig. 3.12.) The number of colonies recovered from wild type strain B2 was represented as $100 \%$. The recovery rate of the mutant varied between 22 and 28 $\%$. 


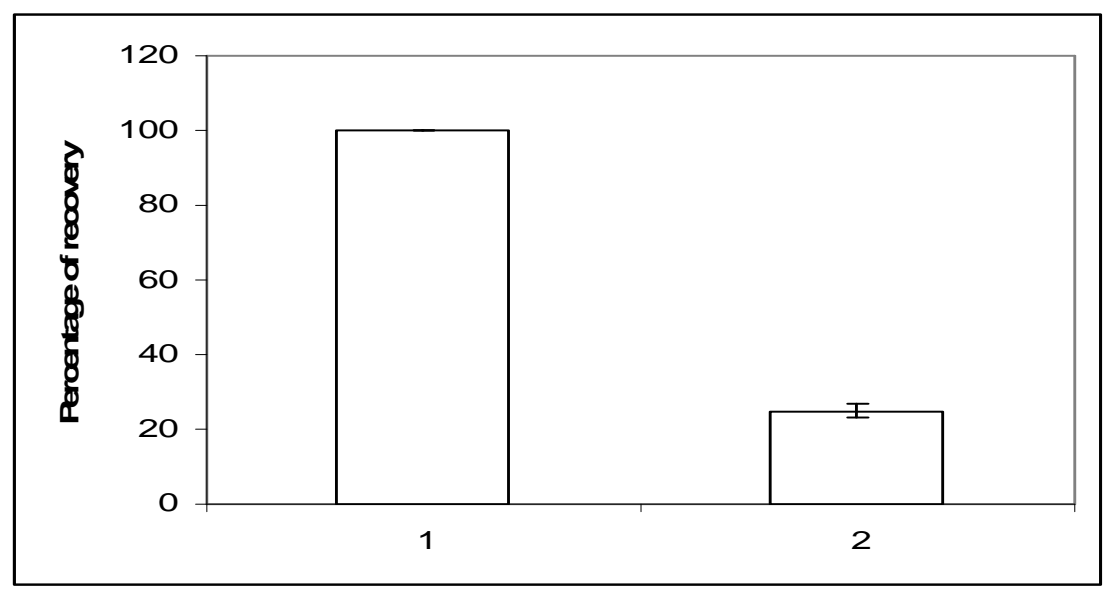

Figure 3.11 Recovery of $\boldsymbol{C}$. jejuni bacteria after invasion assays on Caco2 cells

(1) Parental strain B2, (2) the mutant of B2 carrying a transposon insertion in gene cj0005c (B2 $\Delta c j 0005 c)$. The recovery rate of bacteria from $B 2 \Delta c j 0005 \mathrm{C}$ is clearly reduced compared to the wild type strain. Given the number of colonies reclaimed from strain B2 is defined as $100 \%$, the number of colonies from $B 2 \Delta c j 0005 c$ varied between 22 and $28 \%$ with a mean value of $25 \%$ ( \pm 6.5) and a P-Value of $<0.0001$.

\subsubsection{Construction of a cj0005c knock-out mutant and complementation of the mutant phenotype}

For validation and confirmation of the result described above, homologous recombination was applied to knockout the cj0005c gene in order to verify, that the invasion-deficient phenotype of $C$. jejuni B2 $\Delta c j 0005 C$ was due to the transposon insertion into gene $c j 0005 \mathrm{C}$ and not to other genetic rearrangements somewhere in the genome. A recombinant plasmid carrying the cj0005c gene interrupted by a $\mathrm{kan}^{R}$ gene cassette was introduced into strain B2. Subsequent PCR analysis were performed with genomic DNA from the obtained C. jejuni clones for the detection of an amplicon corresponding of the size of cj0005c including the aphA-3 cassette (B2::cj0005c) (Fig. 3.12b). Using gentamicin protection assays, the knock-out mutant B2::cj0005c confirmed the phenotype of C. jejuni strain B2 harbouring the transposon within cj0005c, which strengthens the finding that the mutation of $c j 0005 c$ is responsible for the invasion-deficient phenotype (Fig. 3.12c). For the restoration of the phenotype of parental strain B2, 
cj0005c was cloned into $C$. jejuni expression plasmid pRRC and introduced into B2::cj0005c to obtain B2::cj0005c-comp-cj0005c (Fig. 3.12a). Afterwards, the invasion assays were repeated with wild-type strain B2, both mutants and the complemented knock-out mutant. The number of B2 colonies recovered was defined as $100 \%$, the mean values of invasion capacities of B2 $\Delta c j 0005 \mathrm{C}$, B2::cj0005c and B2::cj0005c-comp-cj0005c were $25 \%, 29.8 \%$ and $85.6 \%$ respectively. Since the P-values of both mutants were less than 0.0001 , the altered invasion capacities were strongly significant. In contrast, the P-value for the complemented knock-out mutant to be less invasive was only 0.16 , which showed no significant difference compared to the wild-type strain B2. (Fig. 3.12c). 
(a

1

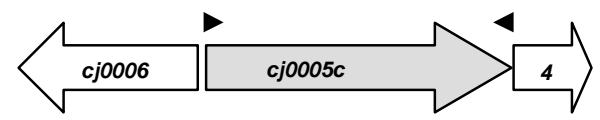

2

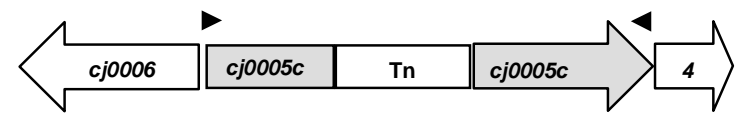

3

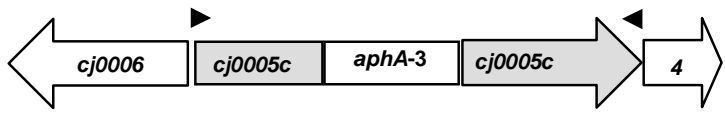

4

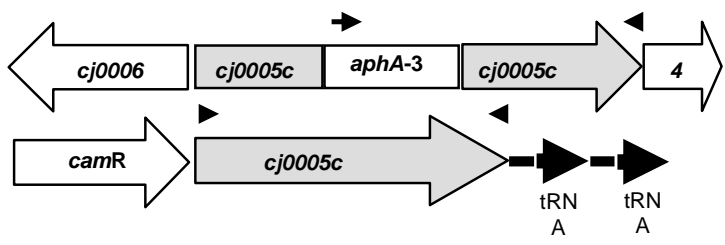

(b

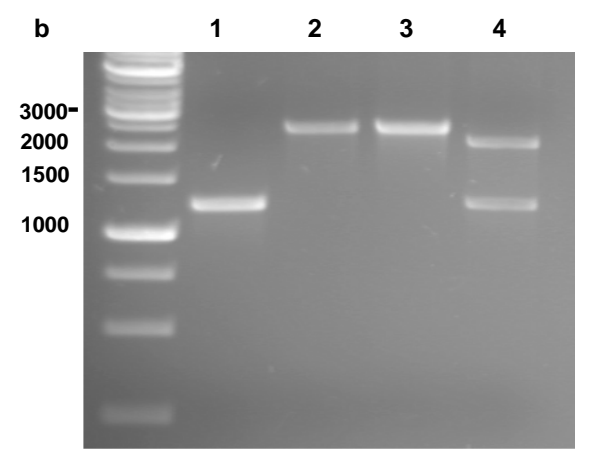

(c

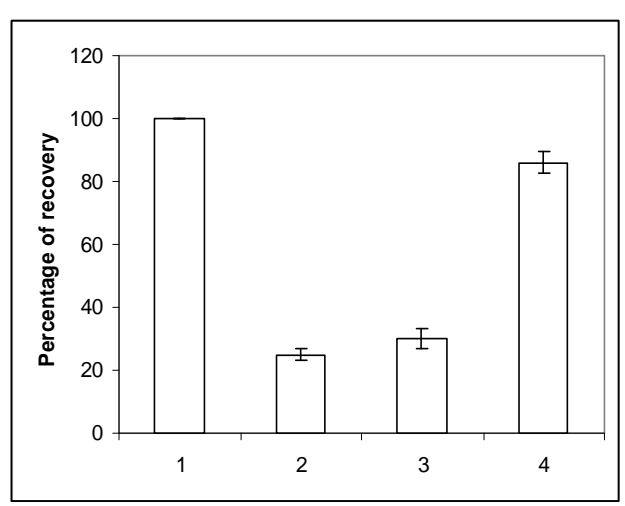

Figure. 3.12 Genome arrangements, verification of cloning procedures and invasion assays of the strains under investigation

(1) parental strain B2. (2) B2 with transposon insertion in cj0005c (B2 $\Delta c j 0005 c)$, (3) cj0005c knock-out mutant (B2::cj0005c), (4) complemented knock-out mutant (B2::cj0005c-comp-cj0005c). (a) Genome disposals of the investigated bacterial strains. Primers Cj0005cF and Cj0005cR for the amplification of $c j 0005 c$ are shown as arrowheads. The primer Kan1 that binds to the 5'-end of the kanamycin resistance cassette is illustrated as an arrow. (b) Detection of the native gene, the respective mutants and the complemented strain by PCR analysis. The native cj0005c gene has a size of $1239 \mathrm{bp}$ (1). Insertion of the transposon and insertion of the kanamycin resistance gene yielded PCR-fragments of 2932 bp (2) and $2623 \mathrm{bp}$ (3), respectively. PCR analysis of the complemented knock out mutant using the primers $\mathrm{Cj} 0005 \mathrm{cF}$ and $\mathrm{Cj} 0005 \mathrm{cR}$ revealed only the amplification of the wild type gene, probably because of its smaller size (not shown). To detect both, the gene with the kanamycin resistance cassette (2234 bp) and the native gene (1239 bp), PCR analysis with primers $\mathrm{Cj} 0005 \mathrm{cF}$ and $\mathrm{Cj} 0005 \mathrm{cR}$ and a third primer (Kan1) corresponding to the 5 '-end of the aphA-3 gene were carried out (4). (c) Gentamicin protection assays. Invasion- 
reduced phenotype of $\mathrm{B} 2 \Delta c j 0005 c$ could be confirmed after specific knock-out of cj0005c (B2::cj0005c). Complementation of the knock-out mutant, in turn, reconstituted the parental phenotype. See text for details.

\subsubsection{The loss of a functional SOR leads to reduced growth in the presence of sodium sulphite}

In order to measure the effect of the SOR knock-out gene product on the proliferation of $C$. jejuni, growth experiments were carried out, both, in the presence and the absence of sodium sulphite. No distinguishable differences were found in the growth of the parental strain B2, the knock-out mutant B2::cj0005c and the complemented mutant in the absence of sodium sulphite. In contrast, in the presence of $10 \mathrm{mM}$ sodium sulphite, the growth of the mutant exhibited a clear reduction compared to B2 and B2::cj0005c-comp-cj0005c (Fig. 3.13).

(a)

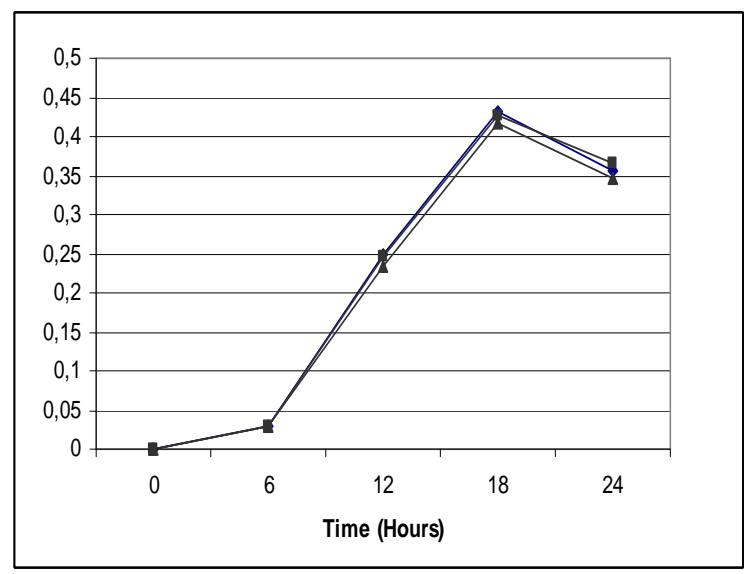

(b)

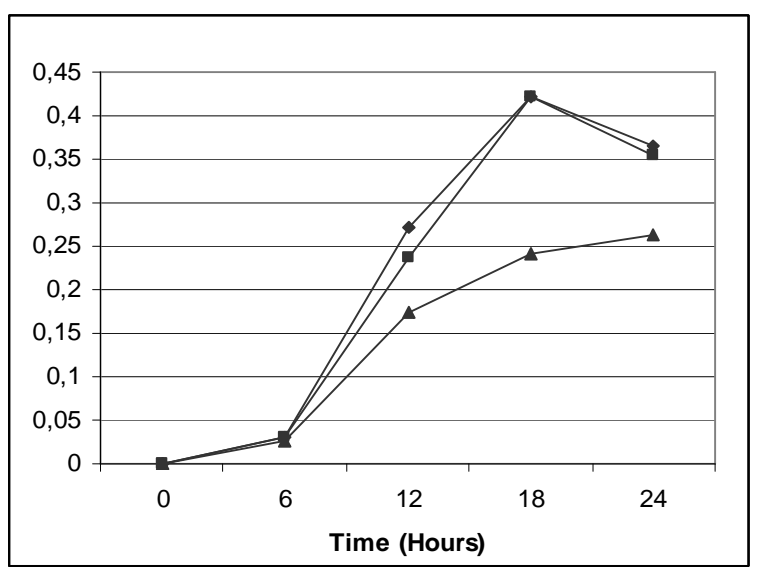

Figure. 3.13 Growth curve in the presence and absence of $10 \mathrm{mM} \mathrm{Na}_{2} \mathrm{SO}_{3}$

Parental strain B2 (\$), B2::cj0005c ( $\mathbf{\Lambda})$ and complemented mutant B2::cj0005c-comp-cj0005c (-) not supplemented (a) and supplemented (b) with $10 \mathrm{mM} \mathrm{Na}_{2} \mathrm{SO}_{3}$. The optical density measured at $600 \mathrm{~nm}$ is shown on the $\mathrm{y}$ axis. 


\subsubsection{The absence of a functional SOR reduces the motility of $C$. jejuni}

As reduced growth in case of an incomplete energy metabolism was detected, It was examined that if the motility of the pathogen was also diminished, since motility is known to be energy consuming. When the motility of the parental strain B2, the knock-out mutant and the complemented knock-out mutant was compared by using semi-solid motility medium, a loss of motility of the mutant B2::cj0005c could be clearly detected which could be reconstituted completely to the wild-type strain motility after introduction of a functional cj0005c gene into the mutant. The results of four independent motility assays performed are summarized in Fig. 3.14a. The mean diameter zone of motility for strain B2 was $40 \mathrm{~mm} \pm 4.6$, while the motility of the mutant $B 2:: c j 0005 c$ was significantly reduced $(18.2 \mathrm{~mm} \pm 1.7$, $\mathrm{P}<0.01)$ compared to B2. After introduction of a functional copy of gene cj0005c, the revertant returned to wild-type motility level with zone diameters of $38.7 \mathrm{~mm} \pm$ 4.1 in average. These findings raised the idea that the reduced infectivity is first of all caused by a lack of motility as a result of a limited energy metabolism.

\subsubsection{Reduced motility conduces to decreased invasiveness}

To investigate whether the reduced motility is the sole reason for the strongly reduced invasion capacity of the cj0005c-deficient mutant or if any other factors are involved, motility was simulated by centrifugation of the bacteria onto the Caco2 host cells. Afterwards, gentamicin protection assays were performed again. Thereby, fixing the number of colonies recovered from wild-type strain B2 as 100 $\%$, we detected $48.6 \%$ of colonies $(\mathrm{P}<0.01)$ after centrifugation of the knock-out mutant B2::cj0005c, while the number of colonies recovered of the complemented mutant was $87 \%$ in average which was not significantly different compared to the wild-type strain (Fig. 3.14b). Compared to the outcome of the gentamicin protection assays without centrifugation (Fig. 3.11) an increase of mutant colonies from $25 \%$ up to $48.6 \%$.was clearly detected This finding was interesting, since one would have expected to shift infectivity of the mutant up to wild-type level after absorbing motility as a virulence factor in case that reduced motility is the only reason for diminished infectivity. But this result indicates that the reduced motility 
is one, but not the only factor responsible for the clearly reduced invasiveness of the cj0005c-deficient mutant.

(a)

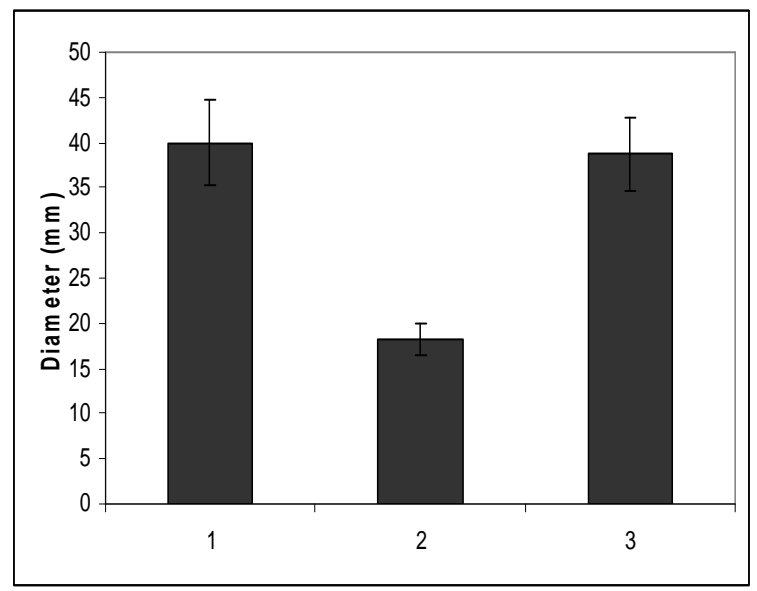

(b)

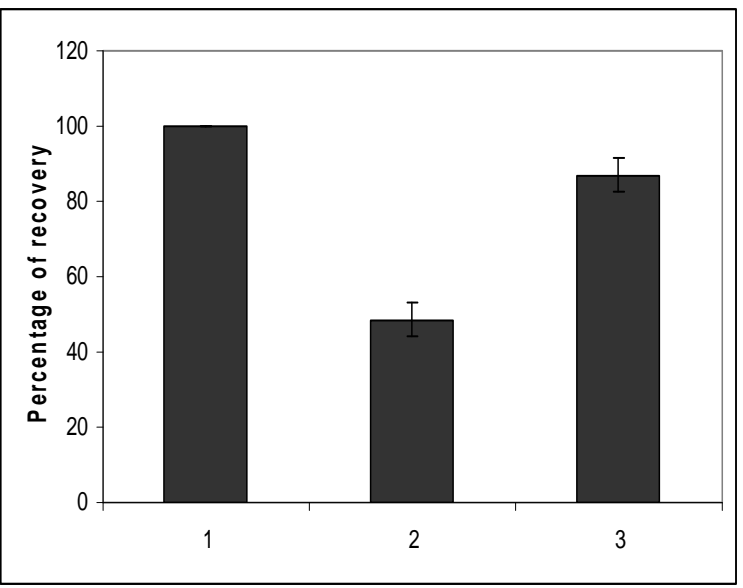

Figure. 3.14 Motility assays and gentamycine protection assays after centrifugation

1. Wild-type strain B2, 2. knock-out mutant B2::cj0005c, 3. complemented mutant B2::cj0005ccomp-cj0005c. (a) Motility assays. The diameter of the motility zones are indicated as bars. The cj0005c-deficient mutant possesses a clearly reduced motility compared to parental strain B2 which could be complemented after introduction of an intact copy of gene cj0005c. (b) Gentamicin protection assays after centrifugation of the bacteria onto Caco2 cells. Defining the number of isolate B2 colonies recovered as $100 \%$, the percentage of colonies of the knock-out mutant $\mathrm{B}:: \mathrm{cj} 0005 \mathrm{c}$ colonies was $48.6 \%$ in average, whereas complementation of the mutant restored the parental phenotype to $87 \%$. See text for details.

\subsubsection{Diminished adherence contributes to reduced invasiveness}

In order to characterize the process of invasion more in detail, It was investigated if already the adherence of the energy deficient mutant is hampered. Therefore, adhesion assays of wild-type isolate B2, the mutant B2::cj0005c and the complemented mutant were performed. To mimic the different motility of the bacteria under investigation, again, the bacteria were centrifuged onto the Caco2 cells. Thereby, an adhesion-deficient phenotype of the mutant could be clearly detected which could be restored to wild-type behaviour after complementation. Taken the number of colonies recovered from parental strain B2 as $100 \%$, the 
mean value of colonies from the mutant B2::cj0005c was $67 \%( \pm 5.1, P<0.0001)$ in contrast to B2::cj0005c-comp-cj0005c with a rate of recovery of $94 \%( \pm 2.2)$ (Fig. 3.15).

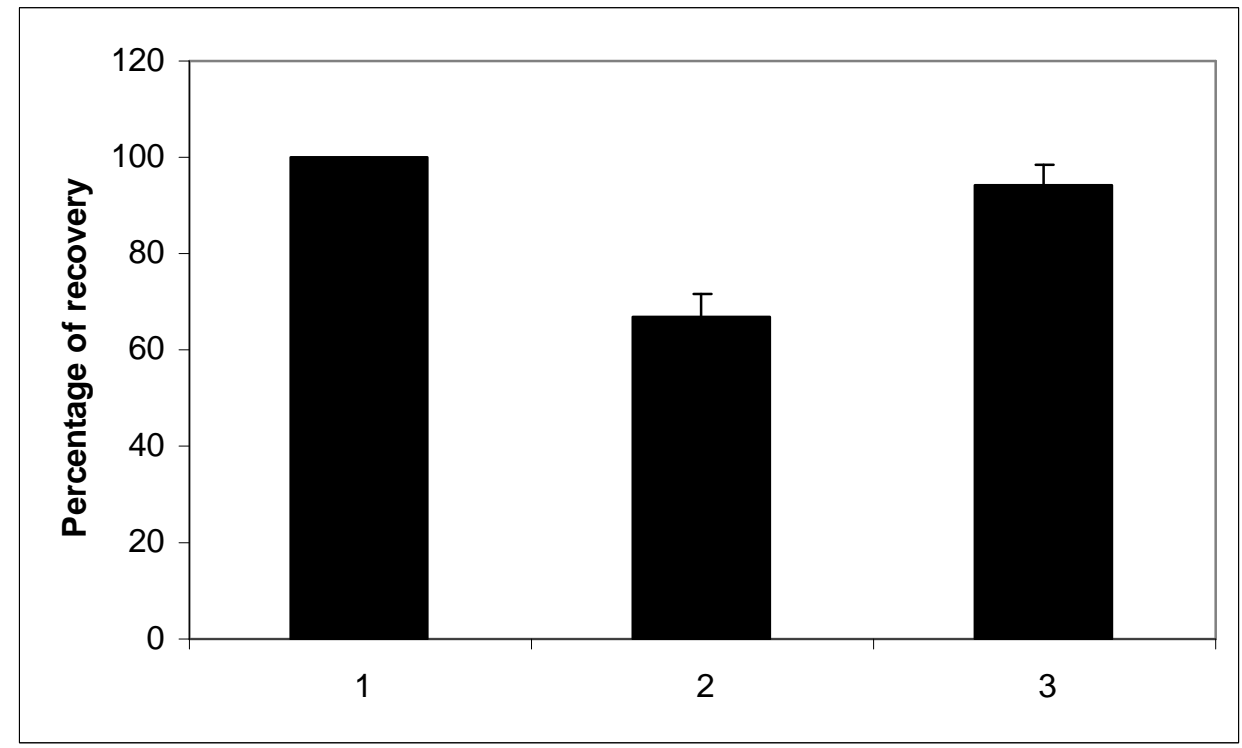

\section{Figure 3.15 Adhesion assay}

1. Wild-type strain B2, 2. knock-out mutant B2::cj0005c, 3. complemented mutant B2::cj0005ccomp-cj0005c. The adherence of the mutant B2::cj0005c onto Caco2 cells was incomplete compared to the wild-type strain. The percentage of adhered colonies of the mutant was only $67 \%$ compared to wild-type isolate B2. See text for details.

\subsubsection{The transcription of virulence associated genes is down-regulated in the absence of a functional SOR}

After the detection that the reduced motility is one, but not the only reason for the decreased invasiveness of the mutant which is deficient in its energy metabolism, the transcription level of genes known to be involved in virulence of the pathogen was examined by semi-quantitative real time RT-PCR. The genes examined were the heat shock proteins groEL and dnaJ (Thies et al., 1999; Konkel et al., 1998), 
the chemotaxis regulator gene cheY (Yao et al., 1997), and the putative chemoreceptor t/p7 (Marchant et al., 2002), also genes known to be crucial for the process of binding and adhesion, like the gene for the fibronectin binding protein cadF (Konkel et al., 1997), as well as genes peb1a and jlpA known to encode adhesion-relevant proteins (Pei and Blaser, 1993; Jin et al., 2001). Furthermore, the transcription level of the Campylobacter invasive antigen ciaB (Rivera-Amill et al., 2001; Konkel et al., 2004), the cytothelial distending toxin cdtB (Pickett and Whitehouse, 1999) and flaA (Guerry et al., 1991) were compared. Furthermore the transcription of genes responsible for O-glycosylation of the flagellum by the synthesis of pseudaminic acid (PseAc) and legionaminic acid (LegAm) was examined (Logan et al., 2008). Thereby, a statistically significant 5.16 fold downregulation of $d n a J$ in the mutant compared to the transcription level of this gene in wild-type strain B2 was clearly detected $(\mathrm{P}<0.001)$. Genes ptmB, ptmC and ptmE, which are involved in the synthesis of legionaminic acid (LegAm) were significantly down-regulated between factor 4.31 to $7.67(\mathrm{P}<0.0001)$ while other genes which are part of the legionaminic acid metabolism were downregulated significantly as well, but to a lesser extent (ptmA, ptmF or ptmG). In contrast, the transcription level of all the other genes studied was not changed significantly irrespective of the presence or absence of SOR. The results are summarized in Table 3.4.The RNA level of mutant complemented with the intact version of cj0005c was examined, it was observed which that the crossing point for the respective genes which were almost identical with the wild type (Table 3.5) 
Table 3.4. Transcription level of selected virulence-associated genes from $C$. jejuni in wildtype strain B2 and mutant B2::cj0005c

Gene

\begin{tabular}{lll} 
B2::cj0005c & B2 wild-type & \\
\hline $\mathrm{Cp}$ (mean value) & $\mathrm{Cp}$ (mean value) & $\begin{array}{l}\text { Fold change } \\
(t \text {-test P-value })\end{array}$
\end{tabular}

Function

\begin{tabular}{|c|c|c|c|c|}
\hline GroEL & $9.79( \pm 0.002)$ & $9.52( \pm 0.02)$ & $1.20(0.03)$ & Heat-shock response \\
\hline DnaJ & $23.34( \pm 0.09)$ & $20.97( \pm 0.05)$ & $5.16(0.0001)$ & Heat shock response \\
\hline DnaK & $12.8( \pm 0.03)$ & $12.64( \pm 0.001)$ & $1.11(0.03)$ & Heat shock response \\
\hline CheY & $12.87( \pm 0.0002)$ & $11.86( \pm 0.87)$ & $2.01(0.13)$ & $\begin{array}{l}\text { Chemotaxis response } \\
\text { regulator }\end{array}$ \\
\hline t/p7 & $6.36( \pm 0.02)$ & $6.66( \pm 0.02)$ & $1.23(0.07)$ & Chemotaxis receptor \\
\hline CadF & $11.70( \pm 0.008)$ & $11.26( \pm 1.15)$ & $1.35(0.51)$ & $\begin{array}{l}\text { Fibronectin binding outer } \\
\text { membrane protein }\end{array}$ \\
\hline peb1a & $9.66( \pm 0.05)$ & $9.78( \pm 0.02)$ & $1.08(0.52)$ & $\begin{array}{l}\text { Periplasmic binding } \\
\text { protein }\end{array}$ \\
\hline JlpA & $13.84( \pm 2.4)$ & $13.44( \pm 1.8)$ & $1.31(0.75)$ & $\begin{array}{l}\text { Surface-exposed } \\
\text { lipoprotein }\end{array}$ \\
\hline CiaB & $20.94( \pm 0.08)$ & $21.49( \pm 0.09)$ & $1.46(0.08)$ & $\begin{array}{l}\text { Campylobacter invasive } \\
\text { antigen B }\end{array}$ \\
\hline$C d t B$ & $13.55( \pm 0.86)$ & $13.84( \pm 0.05)$ & $1.22(0.62)$ & $\begin{array}{l}\text { Cytolethal distending toxin } \\
\text { B }\end{array}$ \\
\hline FlaA & $11.52( \pm 0.04)$ & $11.98( \pm 0.001)$ & $1.37(0.01)$ & Flagellum \\
\hline FlpA & $15.76( \pm 0.27)$ & $15.1( \pm 0.10)$ & $1.58(0.10)$ & Adhesin \\
\hline PorA & $07.23( \pm 0.50)$ & $6.63( \pm 0.66)$ & $1.51(0.058)$ & Adhesin \\
\hline PgIE & $16.39( \pm 0.71)$ & $16.02( \pm 1.03)$ & $1.29(0.64)$ & Protein glycosylation \\
\hline PtmA & $22.29( \pm 0.55)$ & $20.79( \pm 0.17)$ & $2.82(0.0001)$ & LegAm synthesis \\
\hline PtmB & $19.40( \pm 0.77)$ & $16.83( \pm 0.01)$ & $5.93(0.0001)$ & LegAm synthesis \\
\hline PtmC & $18.31( \pm 0.47)$ & $16.20( \pm 1.19)$ & $4.31(0.0001)$ & LegAm synthesis \\
\hline PtmD & $20.53( \pm 0.59)$ & $20.16( \pm 0.72)$ & $1.29(0.39)$ & LegAm synthesis \\
\hline PtmE & $18.11( \pm 0.06)$ & $15.17( \pm 0.38)$ & $7.67(0.0001)$ & LegAm synthesis \\
\hline PtmF & $19.74( \pm 0.50)$ & $18.30( \pm 0.89)$ & $2.71(0.0001)$ & LegAm synthesis \\
\hline PtmG & $19.16( \pm 0.25)$ & $17.64( \pm 0.15)$ & $2.86(0.0001)$ & LegAm synthesis \\
\hline PtmH & $20.17( \pm 0.26)$ & $18.82( \pm 0.12)$ & 2.54. (0.017) & LegAM synthesis \\
\hline PseA & $23.04( \pm 0.48)$ & $21.86( \pm 0.19)$ & $2.26(0.016)$ & PseAc synthesis \\
\hline$P s e B$ & $20.71( \pm 0.02)$ & $21.29( \pm 0.42)$ & $1.49(0.11)$ & PseAc synthesis \\
\hline PseC & $20.74( \pm 0.05)$ & $20.74( \pm 0.016)$ & $0.00(0.80)$ & PseAc synthesis \\
\hline
\end{tabular}




$\begin{array}{lllll}\text { PseD } & 23.30( \pm 0.60) & 21.64( \pm 0.20) & 3.16(0.0001) & \text { PseAc synthesis } \\ \text { PseE } & 21.35( \pm 0.21) & 19.8( \pm 0.05) & 2.92(0.067) & \text { PseAc synthesis }\end{array}$

Strongly downregulated genes are indicated in bold. See text for details.

In contrast, when we compared the transcription level of the genes which are down-regulated in the complemented mutant with the transcription level of the respective genes in the parental strain, the values for the crossing points were almost identical. (Table 3.5).

Table 3.5. Restored transcription levels of genes in the complemented mutant compared to parental strain B2

$\begin{array}{lll}\text { Gene } & \begin{array}{l}\text { B2::cj0005c } \\ \text { comp-cj0005c }\end{array} & \begin{array}{l}\text { Fold change } \\ \text { (Compared to wild-type B2 }\end{array} \\ & \frac{\mathrm{Cp} \text { (mean value) }}{20.87( \pm 0.05)} & \frac{(t \text {-test P-value) }}{1.05(0.571)} \\ \text { ptmA } & 17.45( \pm 0.079) & 1.53(0.071) \\ \text { PtmB } & 16.63( \pm 0.87) & 1.34(0.407) \\ \text { PtmC } & 15.6( \pm 0.20) & 1.34(0.316) \\ \text { PtmE } & 19.74( \pm 0.50) & 2.71(0.014) \\ \text { PtmF } & 17.62( \pm 0.07) & 1.01(0.127)) \\ \text { PtmG } & 22.39( \pm 0.60) & 1.68(0.242) \\ \text { PseD } & 21.11( \pm 0.06) & 1.01(0.071) \\ \text { DnaJ } & \end{array}$

\subsubsection{Reduced autoagglutination of mutant B2::cj0005c}

Recently, Howard and coworkers (2010) reported that $C$. jejuni mutants deficient in gene cj1324 (ptmG) or genes cj1321-1325/6, which are responsible for the legionaminic acid synthesis, showed a significantly decreased capacity for autoagglutination. When the mutant B2::cj0005c was compared with the wild-type strain B2, a significant down-regulation of ptmG-transcription and of other genes of the legionaminic acid metabolism was detected. For this, the competency of autoagglutination of mutant B2::cj0005c compared to wild-type strain B2 was investigated. As is shown in Fig 3.16, the autoagglutination ability of the mutant 
strain is actually diminished. The percent autoagglutination of wild-type strain B2 and complemented mutant was $74.24 \%( \pm 0.25)$ and $73.65 \%( \pm 0.22)$, respectively, whereas the mutant $\mathrm{B} 2:: \mathrm{cj} 0005 \mathrm{c}$ had a remaining autoagglutination level of only $37.02 \%( \pm 0.61)$ which was a significant reduction compared to B2 $(P<0.0001)$.

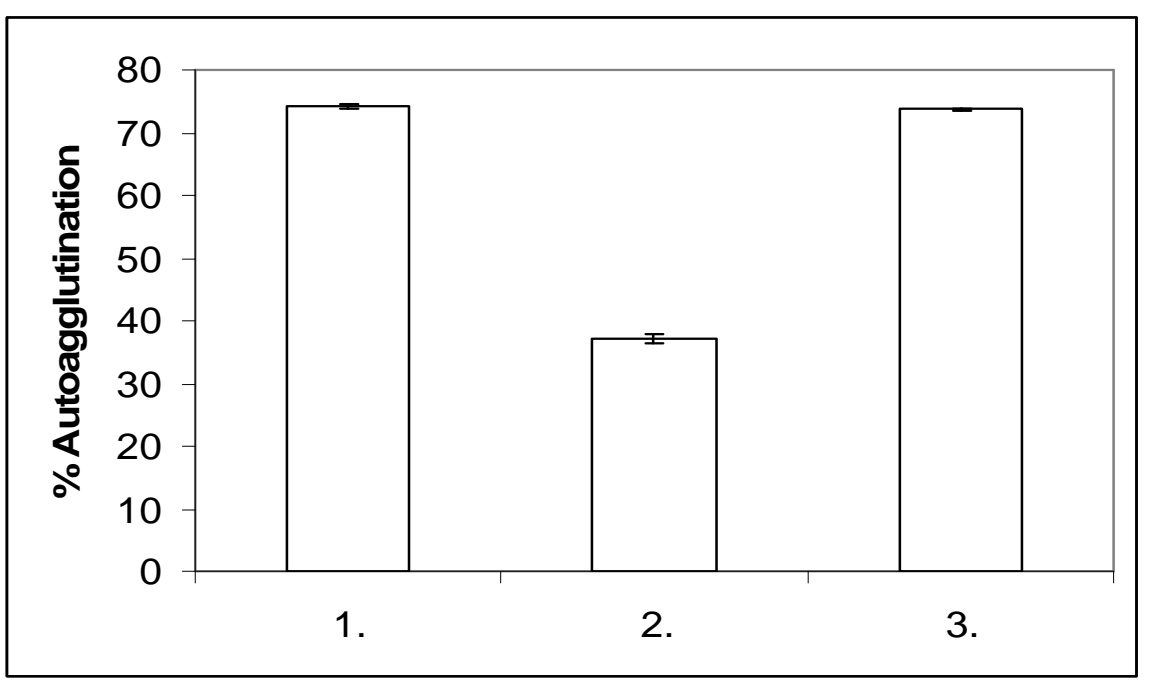

Figure. 3.16. Autoagglutination

Autoagglutination of mutant B2::cj0005c was compared to wild-type strain B2 and complemented mutant. 1. wild-type strain B2, 2. knockout mutant B2::cj0005c, 3. complemented mutant B2::cj0005c-comp-cj0005c. See text for details 


\section{DISCUSSION}

Transposon insertions are interrupting the function of genes and are thus a tool for generating random mutations (Spradlingand and Rubin, 1982). For this, transposon mutagenesis techniques have also been used in $C$. jejuni research to detect genes which contribute to the pathogenicity of the bacterium (Colegio et al., 2001; Golden et al., 2000). Thereby, motility deficient mutants have been examined which carried the transposon in genes that have not been correlated with motility but in genes which belong to the chemotaxis machinery (Golden and Acheson, 2002; Hendrixson et al., 2001). Furthermore, transposon mutagenesis coupled with reporter assays have been used for the identification of genes involved in flageller regulation at the beginning of the flagellar transcription cascade (Hendrixson and DiRita, 2003) and were used to study the nature of Campylobacter colonization in the gastrointestinal tract of chickens (Grant et al., 2005), including the gene encoding the methyl-accepting chemotaxis protein $d_{o c} B$ (tlp 10) (Hendrixson and DiRita, 2004). By using transposon-based mutations

several genes were identified that play an important role for host cell invasion and also for C. jejuni colonization (Novik et al., 2010). Javed and coworkers (2009) used transposon mutagenesis in a hyper-invasive clinical isolate of $C$. jejuni to detect 26 mutants with a clearly reduced invasion capacity. By applying transposon mutagenesis, $C$. jejuni genes which play an important role for the molecular basis of resistance to polymyxin and natural antimicrobial peptides could be identified (Lin et al., 2009).

The present study was undertaken to uncover additional genes of $C$. jejuni that are associated with the invasion process. A total of 660 clones of an existing transposon-based library of clinical $C$. jejuni isolate B2 were screened for reduced invasion of Caco2 cells. Thereby, a clone with a transposon insertion in gene cj0952c, which shows a strong homology to genes representing methyl-accepting chemotaxis proteins (MCPs), was detected. Like all other motile bacteria, C. jejuni has the competence to sense its external environment through chemical 
gradients. Cellular motility and chemotaxis have been connected to virulence of pathogenic bacteria and play a vital role in colonization and invasion of the host intestinal tract (Josenhans and Suerbaum, 2002). Initially, cj0952c was thought to be a pseudogene, since the immediately downstream located gene cj0951c which is encoding a signaling domain, together with $\mathrm{Cj} 0952 \mathrm{c}$, might act as a complete chemoreceptor. Performing BLASTN analysis in the NCBI database, in the $C$. jejuni strains NCTC 11168, CF93-6 and 84-25, the mentioned chemoreceptors is composed of two single genes. Similarly, the genes cj0951c and cj0952c are present separately in the clinical isolate B2. On contrary, in strains $81-176$ or 81116, the corresponding chemoreceptor is the product of only one single gene encompassing the MCP domain, the transmembrane domain and the signaling domain as well. To date, cj0952c has not been described functionally, and was only demonstrated to be up-regulated during the process of chicken colonization (Woodall et al., 2005). As in many other bacteria, C. jejuni resides genes indispensable for chemotaxis like cheA, chew, cheY and MCPs receptors. It is suggested that interaction of $\mathrm{CheW}$ and $\mathrm{CheV}$ is necessary to regulate CheA activity via the MCPs (Rosario et al., 1994). Moreover, it has been shown that $C$. jejuni colonization is only possible with the help of chemotactic-mediated motility (Takata et al., 1992; Yao et al., 1994). Altogether ten genes encoding chemotaxis proteins known as transducer-like proteins (TIps) were identified in the genome of C. jejuni. These ten Tlps can further be classified according to their predicted domain organization into three different groups (A-C) (Marchant et al., 2002). The TIp group $A$ is further divided in subgroups Tlp1, 2, 3, 4, 7 and 10. Thereby, Tlp1 has been demonstrated to be the aspartate chemoreceptor of $C$. jejuni and plays a role in the colonization of the intestinal tract (Hartley-Tassell et al., 2010).

Predicted structures of group A TIps shows classical MCPs organization, identical to that of E. coli MCPs, with a periplasmic sensory domain, which is extremely variable between different receptors, two transmembrane domains, and the Cterminal cytoplasmic signaling domain (Marchant et al., 2002). Cj0952c-Cj0951c (Tlp7) belongs to group A chemoreceptors which are MCPs and having four 
domains that consist of a $\mathrm{N}$-terminal transmembrane domain, a periplasmic ligand-binding domain, a HAMP domain and a C-terminal cytoplasmic signaling domain at the C-terminal end, in which the HAMP domain converts the ligandinduced conformational changes into kinase controlling signals. (Aravind and Ponting, 1999; Butler and Falke, 1998; LeMoual and Koshland, 1996; Williams and Stewart, 1999). Cj0951c shows strong homologies with the cytoplasmic signaling domain of MCPs that possess an adaption region with internal methylation sites. This flexible region mediates the interaction with kinase CheA and chemotaxis protein CheW (Hazelbauer et al., 2008).

In this study, it was shown that transposon insertion in gene cj0952c does not affect the transcription of the adjacent gene cj0951c. In order to create a functional chemoreceptor both genes have probably to be transcribed on the same RNA to allow the aggregation of both subunits to obtain one functionally active chemoreceptor.

To answer the question whether both genes are translated as one protein, the corresponding genes in trans in $C$. jejuni was expressed. For the detection of the recombinant protein a 6x His-tag was added to the C-terminus of Cj0951c. Subsequent western blot analysis only detected a protein that corresponded to the size of Cj0951c but not to the size of a protein that would indicate a protein composed of Cj0952c and Cj0951c. For this, in C. jejuni strain B2, a non-covalent interaction of both subunits might be postulated for the creation of a functional chemoreceptor.

Host-pathogen interaction is a prerequisite for $C$. jejuni pathogenicity and is mediated by the chemotaxis system as it directs the pathogen towards the host cell at the site of entry. Gene alterations in the chemotaxis apparatus reduce the strength of $C$. jejuni to infect human and chicken cells as well. Mutant strains which are deficient in cj0262 (Tlp4, docC) or cj0019 (Tlp10, docB) revealed a decreased colonization of the chicken intestinal cells ( Hendrixson and DiRita, 
2004). In additional, the chemoreceptors Tlp1 (cj1506), Tlp2 (cj0144), Tlp3 (cj1564), Tlp4 and Tlp10 are important for the disease-causing ability of the invading pathogen (Golden and Archeson, 2002; Hendrixson and DiRita, 2004; Vegge et al., 2009). However, less is known about the chemoreceptors of $C$. jejuni, so far.

Recently, Cj1506c (TIp1) of C. jejuni was identified as the aspartate chemosensory receptor and was the first chemoreceptor of this pathogen described with respect to its particular ligand (Hartley et al., 2010). In this study it has described that the inactivation of gene cj0952c changes the chemotactic behaviour of $C$. jejuni with regard to formic acid. While in the wild-type strain formic acid was clearly a chemoattractant, the mutant showed a biphasic halo together with an inner repulsion zone which was surrounded by a diminished ring. The reduced, but still measurable attraction of the mutant towards formic acid might be mediated by the energy taxis system of the pathogen which consists of the two proteins CetA and CetB that act together as energy taxis receptors. In this system, changes in the redox state of the electron transport chain are sensed by Cet $B$ and are subsequently relayed to Cet $A$ via direct interaction. CetA further transduces the signal to the chemotactic machinery and leads to changes in the direction of motility (Hendrixson et al., 2001; Elliot and DiRita, 2008). Energy taxis has been shown to direct $C$. jejuni towards attractants which serve as carbon sources, electron acceptors and, as in the case of formic acid, electron donors (Vegge et al., 2009). The formation of an inner repulsion zone might suggest that another chemosensory mechanism exists that recognizes formic acid as a chemorepellent.

The enzyme formate dehydrogenase catalizes the oxidation of formate to $\mathrm{CO}_{2}$, protons and electrons which are injected into the respiratory chain and, thus, serves as an electron donor. The enzyme consists of four subunits which are encoded by the $f d h A B C D$ operon of $C$. jejuni encompassing the genes cj1511ccj1508c (Weerakoon et al., 2009). In this study it was shown that the $C$. jejuni 
chemoreceptor for the detection formic acid is composed by the proteins Cj0952c and Cj0951c. To investigate whether this unusual composition of the chemoreceptor is going along with the utilization of its particular chemoattractant, the growth curves in the presence and absence of formic acid was carried out. Thereby any differences in the proliferation of wild-type strain B2, the mutant $\mathrm{B} 2 \Delta c j 0952 \mathrm{c}$ and the complemented mutant was not detected. Interestingly, the growth of the bacteria in general (except of B2 $\Delta f d h$ ), significantly promoted in the presence of formic acid in medium, indicating that the generation of electrons by the oxidation of formic acid is an important process in the context of the energy metabolism of $C$. jejuni.

Many prokaryotes possess a complex electron transport chain to cope their energy requirement. Similarly, $C$. jejuni has a highly complex respiratory chain that allows the pathogen to use a variety of electron donors like, succinate, malate, Dlactate, hydrogen, formate, $\mathrm{NAD}(\mathrm{P}) \mathrm{H}$ and sulphite (Kelly, 2001; Sellars et al., 2002; Myers and Kelly, 2005; Weerakoon et al., 2009). In C. jejuni resides an enzyme named molybdenum-containing sulphite: cytochrome c oxidoreductase (SOR) which is involved in the oxidation of sulphite to sulphate and the release of electrons which are used as fuel in the respiratory chain. SOR is the ultimate product of two genes including cj0005c and cj0004c and has similarity to the corresponding enzyme of Starkeya novella (Jonathan and David, 2005). Gene cj0004c is responsible for encoding the monohaem cytochrome c subunit, whereas cj0005c encodes the molybdopterin oxidoreductase. Both subunits of the SOR work in a unifunctional manner and catalyze the conversion of sulphite to sulphate in the presence of oxygen to generate electrons which are used in the respiratory chain after the $b_{1} c_{1}$ complex at the level of cytochrome $c$ (Kappler et al., 2000; Kappler and Dahl, 2001; Myers and Kelly, 2005).

In this work, a cj0005c-deficient mutant of $C$. jejuni with strongly diminished motility function of the pathogen and, therefore, a reduced ability to invade the host cells was investigated, which, in contrast to other motility deficient strains, is 
limited in its energy metabolism instead of the flagellar apparatus. C. jejuni motility function is the key regulator to infect the assigned host, so we can say that motility is the main player responsible for $C$. jejuni colonization. In the recent years, several studies have documented the impact of genes and gene products of the flagellar apparatus with particular reference to the colonization or invasion of host cells. Morooka and coworkers (1985) described that motility-deficient strains of $C$. jejuni were eliminated from the intestinal tract of suckling mice, while a motilitycompetent strain colonized the lower portion of the intestine, the caecum as well as the colon. Pathogen colonization in this animal was shown to depend on the flagella, because a non-flagellated mutant was not able to survive inside the rabbit intestine (Pavlovskis et al., 1991). In addition, the chicken intestinal tract was shown to be invaded only by sound motile strains of $C$. jejuni, reflecting an intact flagellum to be an important invading factor of the pathogen to cause disease (Nachamkin et al., 1993). Mutants can be generated by homologous recombination procedures which can be subsequently investigated in in vitro cell culture experiments for their ability to infect the host cells. Thereby, the role of motility in the invasiveness of $C$. jejuni could also be established (Wassenaar et al., 1991; Yao et al., 1994; Szymanski et al., 1995; Konkel et al., 2004). In addition, numbers of coding sequences and gene products have been described that attributed to the flagellum function for successful motility which is further responsible for motility-mediated pathogen infectivity (Hendrixson, 2008).In order to exclude motility as a virulence factor, the wild-type strain B2, the mutant and the complemented mutant were centrifuged directly onto the host cells. Interestingly, the infectivity of the mutant up to $48.6 \%$ was only restore compared to the parental strain, which clearly indicated that motility was only one, but not the only reason for the incomplete infectivity of the mutant. For this, it was investigated whether the energy-deficient genotype of the mutant has some effect on the transcription level of other virulence-related genes. Semi-quantitative real time RT-PCR was used to investigate selected genes associated with stress response, motility, chemotaxis as well as binding and adhesion. In addition, genes essential for the invasion of host cells by $C$. jejuni were examined. As a result of 
these investigations, a significant down-regulation of dnaJ and of genes for the synthesis of legionaminic acid in the mutant compared to parental strain B2 was detected.

In most prokaryotes and eukayotes the DnaJ (Hsp 40) proteins reside as cochaperones of their corresponding heat shock protein 70 (Hsp 70) partners (DnaKs) (Genevaux et al., 2007). The DnaJ family proteins have an N-terminal Jdomain of approximately 70 amino acids (Hennessy et al., 2005). The molecular function of DnaJ is the interaction of the substrate-bound cochaperone with its corresponding ATP-bound DnaK protein via J-domain and ATPase domain, which mediates a subsequent conformational change of DnaK. Conformational change of DnaK, in turn, stimulates ATP-hydrolysis and is leading to high affinity and low exchange rates for its particular substrate (Liberek et al., 1991; Karzai et al., 1996; Laufen et al., 1999). DnaJ co-chaperones in $C$. jejuni were shown to be an integral part of the heat shock response of the invader (Konkel et al., 1998). The DnaJ protein sequences of $C$. jejuni exhibit $60.8 \%$ homology with the corresponding protein of $E$. coli. It is also established that the function of an E. coli dnaJ deletion mutant could be restored by complementing the corresponding gene from C. jejuni strain F38011 (Konkel et al., 1998).

The acetoamidino form of legionaminic acid (LegAm) together with pseudaminic acid (PseAc) are O-linked to the flagellin protein of most $C$. jejuni strains (Logan et al., 2008). Previously, eight genes have been reported to be involved in the legionaminic acid synthetic pathway including cj1324 (ptmG), cj1325/6 (ptmH), cj1327-cj1330 (ptmC, ptmD, ptmE, ptmF), cj1331 (ptmB) and cj1332 (ptmA) gene (McNally et al., 2007). Although the role of O-glycosylation by legionaminic acid is still not well defined, recent investigations have given a first insight into its biological relevance (Howard et al., 2010). In their report they describe a cj1324deficient mutant, which is fully motile but has a decreased autoagglutination and biofilm formation due to the lack of two legionaminic acid glycan modifications. Furthermore, this mutant possesses a diminished activity to invade chicken cells. 
When the transcription level of the LegAm genes in the cj0005c mutant was investigated, only a 2.86 fold down-regulation of cj1324 (ptmG) was documented. In this study a reduced autoagglutination was shown, but the formation of biofilm was not affected (data not shown). In contrast to the study from Howard et al., (2010) the transcription level of all genes known to be involved in the synthesis of legionaminic acid was examined. Thereby a strong down-regulation of ptmB (cj1331), ptmC (cj1327) and ptmE (1329), in the cj0005c-deficient mutant was detected and a weaker down-regulation of genes ptmA (cj1332), ptmF (cj1330), and ptmG (cj1324) compared to wild-type strain B2. Although transcription analysis was not carried out that covered the whole genome status of the pathogen, alterations in the transcription level of other genes are likely. Nevertheless, the results were obtained so far might indicate a correlation of the affected biological properties of the mutant (e.g. diminished adhesion and autoagglutination) with the downregulation of genes of the LegAm metabolism.

Taken together, two factors of $C$. jejuni that contribute to host cell invasion by two different mechanisms was identified. In the case of cj0952c, a chemoreceptor subunit is deleted, which probably reduces the proper accession of $C$. jejuni to its host cell by a restricted response towards the chemoattractant formic acid and, consequently, by reduced motility. In contrast, the cj0005c-deficient mutant is limited in motility and adhesion probably due to its narrowed energy metabolism. 


\section{References}

Altekruse S.F., Stern N.J., Fields P.I. and Swerdlow D.L. (1999). Campylobacter jejuni-an emerging foodborne pathogen. Emerg Infect Dis. 5:2835

Asbury A.K. and Cornblath D.R. (1990). Assessment of current diagnostic criteria for Guillain-Barré syndrome. Ann Neurol. 27:S21-4.

Asbury A.K., Arnason B.G. and Adams R.D. (1969). The inflammatory lesion in idiopathic polyneuritis. Its role in pathogenesis. Medicine. 48:173-215.

Asrat D., Hathaway A. and Ekwall E. (1999). Studies on enteric campylobacteriosis in Tikur Anbessa and Ethio-Swedish children's hospital, Addis Ababa, Ethiopia. Ethiop Med J. 37:71-84.

Aravind I. and Ponting C.P. (1999). The cytoplasmic helical linker domain of receptor histidine kinase and methyl-accepting proteins is common to many prokaryotic signalling domains. FEMS Microbiol Lett. 176:111-116.

Banmali P., Fritschi L. and Atrie J. (2006). Epidemiology of notified campylobacteriosis in Western Australia. Nepal Med Coll J. 8:31-35.

Berg R.D. (1983). Translocation of indigenous bacteria from the intestinal tract, p. 333-352. In D.J. Hentges (ed.), Human intestinal microflora in health and disease. Academic Press, Inc., New York.

Birk T., Ingmer H., Andersen M.T., Jorgensen K. and Brondsted L. (2004). Chicken juice, a food-based model system suitable to study survival of Campylobacter jejuni. Lett. Appl. Microbiol. 38:66-71. 
Bras A.M., Chatterjee S., Wren B.W., Newell D.G. and Ketley J.M. (1999). A novel Campylobacter jejuni two-component regulatory system important for temperature-dependent growth and colonization. J Bacteriol. 181:3298-3302.

Butler S.I. and Falke J.J. (1998). Cysteine and disulfide scanning reveals two amphiphilic helices in the linker region of the aspartate chemoreceptor. Biochemistry. 37:10746-10756.

Bryan F.L. and Doyle M.P. (1995) Health risks and consequences of Salmonella and Campylobacter jejuni in raw poultry. J Food Prot. 58:326.

Byrne G.I. and Moulder J.W. (1978). Parasitespecified phagocytosis of Chlamydia psittaci and Chlamydia trachomatis by $\mathrm{L}$ and HeLa cells. Infect. Immun. 19: 598-606.

Chaveerach P., ter Huurne A.A., Lipman L.J. and van Knapen F. (2003). Survival and resuscitation of ten strains of Campylobacter jejuni and Campylobacter coli under acid conditions. Appl Environ Microbiol. 69:711-714.

Christiana.S.V., Brondsted L., Li Y.P., Bang B.P. and Ingmer H. (2009) Energy taxis drives Campylobacter jejuni toward the most favorable conditions for growth. Applied and Environment Microbiology. 75:5308-14.

Clausen J.D., Christiansen G., Holst H.U. and Birkelund S. (1997). Chlamydia trachomatis utilizes the host cell microtubule network during early events of infection. Mol. Microbiol. 25:441-449. 
Colegio O.R., Griffin IV T.J., Grindley N.D.F. and Galán J.E. (2001). In vitro transposition system for efficient generation of random mutants of Campylobacter jejuni. J Bacteriol. 183:2384-2388.

Cortes-Bratti X., Chaves-Olarte E., Lagergård T. and Thelestam M. (1999). The cytolethal distending toxin from the chancroid bacterium Haemophilus ducreyi induces cell-cycle arrest in the G2 phase. J. Clin. Investig. 103:107-115.

Dasti J.I., Groß U., Pohl S., Lugert R., Weig M. and Schmidt-Ott R. (2007). Role of plasmid-encoded tet(O) gene in tetracycline-resistant clinical isolates of Campylobacter jejuni and Campylobacter coli. J Med Microbiol 56:833-837.

Doyle M.P. and Roman D.J. (1982). Response of Campylobacter jejuni to sodium chloride. Appl Environ Microbiol. 43:561-565.

Everest P.H., Goossens H., Butzler J.P., Lloyd D., Knutton S., Ketley J.M. and Williams P.H. (1992). Differentiated Caco-2 cells as a model for enteric invasion by Campylobacter jejuni and C. coli. J Med Microbiol. 37:319-325.

Elliot K T. and DiRita V.J. (2008). Characterization of CetA and CetB, a bipartite energy taxis system in Campylobacter jejuni. Mol Microbiol. 69:1091-1103.

FauchEre J.L., Veron M., Lellouch-Tubiana A. and Pfister A. (1985). Experimental infection of gnotobiotic mice with Campylobacter jejuni: colonization of intestine and spread to lymphoid and reticulo-endothelial organs. J. Med. Microbiol. 20:215-224.

Fauchere J.L., Rosenau A., Veron M., Moyen E.N., Richard S. and Pfister, A. (1986). Association with HeLa cells of Campylobacter jejuni and Campylobacter coli isolated from human feces. Infect Immun. 54:283-287. 
Fox J.G., Rogers A.B., Whary M.T., Ge Z., Taylor N.S., Xu S., Horwitz B.H. and Erdman S.E. (2004). Gastroenteritis in NF-kappaB-deficient mice is produced with wild-type Camplyobacter jejuni but not with $C$. jejuni lacking cytolethal distending toxin despite persistent colonization with both strains. Infect Immun 72: 1116-1125.

Friedman C.R., Neimann J., Wegener H.G. and Tauxe R.V. (2000). Epidemiology of Campylobacter jejuni infections in the United States and other industrialized nations. In: Nachamkin I., Blaser M.J., (Eds). Campylobacter. ASM Press, Washington, pp. 121-39.

Galan J.E. and Collmer A. (1999). Type III secretion machines: bacterial devices for protein delivery into host cells. Science. 284:1322-1328.

Genevaux P., Georgopoulos C. and Kelley W.L. (2007). The Hsp70 chaperone machines of Escherichia coli: a paradigm for the repartition of chaperone functions. Mol. Microbiol. 66:840-857.

Goon S., Kelly J.F., Logan S.M., Ewing C.P. and Guerry P. (2003).Pseudaminic acid, the major modification on Campylobacter is synthesized via the cj1293 gene. Mol Microbial. 50:659-71.

Goon S., Ewing P.C., Lorenzo M., Pattarini D., Majam G. and Guerry P. (2006). A sigma28-regulated nonflagella gene contributes to virulence of Campylobacter jejuni 81-176. Infect. Immun. 74:769-772.

Golden N.J., Camilli A. and Acheson D.W. (2000). Random transposon mutagenesis of Campylobacter jejuni. Infec. Immun. 68:5450-5453. 
Golden N.J. and Acheson D.W. (2002). Identification of motility and autoagglutination Campylobacter jejuni mutants by random transposon mutagenesis. Infect Immun. 70:1761-1771.

Govoni V. and Granieri E. (2001). Epidemiology of the Guillain-Barré syndrome. Curr Opin Neurol. 14:605-13.

Grant A.J., Coward C., Jones M.A., Woodall C.A., Barrow P.A. and Maskell D.J. (2005). Signature-tagged transposon mutagenesis studies demonstrate the dynamic nature of cecal colonization of 2-week-old chickens by Campylobacter jejuni. Appl. Environ. Microbiol. 71:8031-8041.

Griffin J.W., Li C.Y. and Ho T.W. (1996). Pathology of the motor-sensory axonal Guillain-Barré syndrome. Ann Neurol. 39:17-28.

Guerry P., Alm R.A., Power M.E., Logan S.M. and Trust T.J. (1991). Role of two flagellin genes in Campylobacter motility. J. Bacteriol 173:4757-4764.

Guerry P., Ewing C.P., Schirm M., Lorenzo M., Kelly J., Pattarini D., Majam G., Thibault P. and Logan S. (2006). Changes in flagellin glycosylation affect Campylobacter autoagglutination and virulence. Mol. Microbiol. 60:299-311.

Hartley Tassell L.E., Shewell L.K., Day C.J., Wilson J.C., Sandhu R., Ketley J.M. and Koroloik V. (2010). Identification and characterization of the aspartate chemosensory receptor of Campylobacter jejuni. Mol Microbiol. 75:710-730.

Hazelbauer G.L., Falke J.J. and Parkinson J.S. (2008). Bacterial chemoreceptors: high-performance signalling in network arrays. Trends Biochem Sci. 33:9-19. 
Hassane D.C., Lee R.B., Mendenhall M.D. and Pickett C.L. (2001). Cytolethal distending toxin demonstrates genotoxic activity in a yeast model. Infect. Immun. 69:5752-5759.

Hazeleger W.C., Wouters J.A., Rombouts F.M. and Abee T. (1998). Physiological activity of Campylobacter jejuni far below the minimal growth temperature. Appl Environ Microbiol. 64:3917

Hendrixson D.R., Akerley B.J. and DiRita V.J. (2001). Transposon mutagenesis of Campylobacter jejuni identifies a bipartite energy taxis system required for motility. Mol. Microbiol. 40:214-224.

Hendrixson D.R. and DiRita V.J. (2003). Transcription of sigma54-dependent but not sigma28-dependent flagellar genes in Campylobacter jejuni is associated with formation of the flagellar secretory apparatus. Mol. Microbiol. 50:687-702.

Hendrixson D.R. and DiRita V.J. (2004). Identification of Campylobacter jejuni genes involved in commensal colonization of the chick gastrointestinal tract. Mol. Microbiol. 52:471-484.

Hendrixson D.R. (2008). Regulation of flagellar gene expression and regulation. In Campylobacter, $3^{\text {rd }}$ edn pp 545-558. Edited by I. Nachamkin, C. M. Szymanski and M. J. Blaser. Washington DC: American Society for Microbiology.

Hennessy F., Nicoll W.S., Zimmermann R., Cheetham M.E. and Blatch G.L. (2005). Not all $\mathrm{J}$ domains are created equal: implications for the specificity of HSP40-Hsp70 interactions. Protein Sci. 14:1697-1709.

Heywood W., Henderson B., and Nair S.P. (2005). Cytolethal distending toxin: creating a gap in the cell cycle. J. Med. Microbiol. 54:207-216. 
Howard S.L., Jagannathan A., Soo E.C., Hui J.P.M., Aubry A.J., Ahmed I., Karlyshev A., Kelly J.F., Jones M.A., Stevens M.P., Logan S.M. and Wren B.W. (2010). Campylobacter jejuni glycosylation island important in cell charge, legionaminic acid biosynthesis, and colonization of chickens. Infect Immun 77:2544-2556.

Hugdahl M.B., Beery J.T. and Doyle M.P. (1988). Chemotactic behavior of Campylobacter jejuni. Infect. Immun. 56:1560-1566

Javed M.A., Grant A.J., Bagnall M.C., Maskell D.J., Newell D.G. and Manning G. (2009). Transposon mutagenesis in a hyper-invasive clinical isolate of Campylobacter jejuni reveals a number of genes with potential roles in invasion. Microbiology. 156:1134-43.

Jin S., Joe A., Lynett J., Hani E.K., Sherman P. and Chan V.L. (2001). JlpA, a novel surface-exposed lipoprotein specific to Campylobacter jejuni, mediates adherence to host epithelial cells. Mol Microbiol 39:1225-1236.

Johnson W.M. and Lior H. (1988). A new heat-labile cytolethal distending toxin (CLDT) produced by Campylobacter spp. Microb. Pathog. 4:115-126.

Jonathan D.M. and David J.K. (2005). A sulphite respiration system in the chemoheterotrophic human pathogen Campylobacter jejuni. Microbiology $151: 233-242$

Josenhans C. and Suerbaum S. (2002). The role of motility as a virulence factor in bacteria. Int J Med Microbiol. 291:605-616.

Karlyshev A.V. and Wren B.M. (2005). Development and application of an insertional system for gene delivery and expression in Campylobacter jejuni. Appl Environ Microbiol.71:4004-4013. 
Kappler U., Bennett B., Rethmeier J., Schwarz G., Deutzmann R., McEwan A. G. and Dahl C. (2000). Sulfite:cytochrome c oxidoreductase from Thiobacillus novellus. J. Biol. Chem. 275:13202-13212

Kappler U. and Dahl C. (2001). Enzymology and molecular biology of prokaryotic sulfite oxidation. FEMS Microbiol. Lett. 203:1-9.

Karzai A.W. and McMacken R. (1996). A bipartoite signaling mechanism involved in DnaJ-mediated activation of the Escherichia coli DanK protein. J Biol Chem. 271:11236-11246.

Kelly D.J. (2001). The physiology and metabolism of Campylobacter jejuni and Helicobacter pylori. J Appl Microbiol. 90:16S-24S.

Konkel M.E. and Joens L.A. (1989). Adhesion to and invasion of HEp-2 cells by Campylobacter spp. Infect Immun. 57:2984-2990.

Konkel M.E., Hayes S.F., Joens L.A. and Cieplak W.Jr. (1992). Characteristics of the internalization and intracellular survival of Campylobacter jejuni in human epithelial cell cultures. Microb. Pathog. 13:357-370.

Konkel M.E., Mead D.J., Hayes S.F. and Cieplak W. (1992a) Translocation of Campylobacter jejuni across human polarized epithelial cell monolayer cultures. J. Infect. Dis. 166:308-315.

Konkel M.E., Garvis S.G., Tipton S.L., Anderson Jr. D.E. and Cieplak Jr W. (1997). Identification and molecular cloning of a gene encoding a fibronectinbinding protein (CadF) from Campylobacter jejuni. Mol Microbiol 24:953-963. 
Konkel M.E., Kim B.J., Klena J.B., Young C.R. and Ziprin R. (1998). Characterization of the thermal stress response of Campylobacter jejuni. Infect Immun. 66:3666-72.

Konkel M.E., Kim B.J., Rivera-Amill V. and Garvis S.G. (1999). Bacterial secreted proteins are required for the internalization of Campylobacter jejuni into cultured mammalian cells. Mol. Microbiol. 32:691-701.

Konkel M.E., Klena J.D., Rivera-Amill V., Monteville M.R., Biswas D., Raphael B. and Mickelson J. (2004). Secretion of virulence proteins from Campylobacter jejuni is dependent on a functional flagellar export apparatus. J. Bacteriol. 186:3296-3303.

Kopecko D.J., Lan Hu and Kristien J.M.Z. (2001). Campylobacter jejunimicrotubule dependent invasion. Trends in Microbiology 9:389-396

Kops S.K., Lowe D.K., Bement W.M. and West A.B. (1996). Migration of Salmonella typhi through intestinal epithelial monolayers: an in vitro study. Microbiol Immunol. 40:799-811.

Lai-King N.G., Sherburne R., Taylar D.E. and Stiles M.E (1985). Morphological forms and viability of Campylobacter species studied by electron microscopy. $J$ of Bacteriology. 64:338-343

Lara-Tejero M. and Galán J.E. (2000). A Bacterial Toxin That Controls Cell Cycle Progression as a Deoxyribonuclease I-Like Protein. Science. 290:354-357

Lara-Tejero M. and Galan J.E. (2001). CdtA, CdtB, and CdtC form a tripartite complex that is required for cytolethal distending toxin activity. Infect Immun. 69:4358-4365. 
Laufen T., Mayer M.P., Beisel C., Klostermeier D., Mogk A., Reinstein J. and Bukau B. (1999). Mechanism of regulation of hsp70 chaperones by DnaJ cochaperones. Proc Natl Acad Sci USA. 96:5452-5457.

Le Moual H. and Koshland Jr D.E. (1996). Molecular evolution of the C-terminal cytoplasmic domain of a superfamily of bacterial receptors involved in taxis. $J \mathrm{Mol}$ Biol. 261:568-585.

Lecuit M., Abachin E., Martin A., Poyart C., Pochart P., Suarez F., Bengoufa D., Feuillard J., Lavergne A., Gordon J.I., Berche P., Guillevin L. and Lortholary O. (2004). Immunoproliferative small intestinal disease associated with Campylobacter jejuni, N. Engl. J. Med. 350:239-248

Lee S.H., Butler S.M. and Camilli A. (2001). Selection for in vivo regulators of bacterial virulence. Proc Natl Acad Sci USA. 98:6889-6894.

Levine M.M., Kaper J.B., Black R.E. and Clements M.L.(1983). New knowledge on pathogenesis of bacterial enteric infections as applied to vaccine development. Microbiol. Rev. 47:510-550.

Liberek K., Marszalek J., Ang D., Georgopoulos C. and Zylicz M. (1991). Escherichia coli DnaJ anf GrpE heat shock proteins jointly stimulate ATPase activity of Dank. Proc Natl Acad Sci USA. 88:2874-2878.

Lin J., Wang Y. and Hoang K.V. (2009). Systematic identification of genetic loci required for polymyxin resistance in Campylobacter jejuni using an efficient in vivo transposon mutagenesis system. Foodborne Pahtog Dis. 6:173-185.

Lisa D.K., Douglas I.G. and Andre G.B.T. (2009). Campylobacter jejuni induces transcellular translocation of commensal bacteria via lipid rafts Gut Pathogens 3:1(1):2. 
Logan S.M., Schoenhofen I.C. and Guerry P. (2008). O-linked flagellar glycosylation in Campylobacter, p.471-481. In I. Nachamkin, C. M. Scymanski, and M. J. Blaser (ed), Campylobacter, $3^{\text {rd }}$ ed. ASM Press, Washington, DC.

MacCallum A., Hardy S.P. and Everest P.H. (2005). Campylobacter jejuni inhibits the absorptive transport functions of Caco-2 cells and disrupts cellular tight junctions. Microbiology. 151:2451-2458.

Marchant J., Wren B. and Ketley J. (2002). Exploiting genome sequences: predictions for mechanisms of Campylobacter chemotaxis. Trends Microbiol. 10:155-159.

Ma Y., Hanning I. and Slavik M. (2009). Stress-induced adaptive tolerance response and virulence gene expression in Campylobacter jejuni. J Food Safety. 29:126-143.

McKhann G.M., Cornblath D.R., Ho T.W., Li C.Y., Bai A.Y. and Wu H.S. (1991). Clinical and electrophysiological aspects of acute paralytic disease of children and young adults in northern China. Lancet. 7:593-7.

McNally D.J., Aubry A.J., Hui J.P., Khieu N.H., Whitfield D., Ewing C.P., Guerry P., Brisson J.R., Logan S.M. and Soo E.C. (2007). Targeted metabolomics analysis of Campylobacter coli VC167 reveals legionaminic acid derivates as novel flagellar glycans. J Biol Chem 282:14463-14475.

McSweegan E. and Walker R.I. (1986). Identification and characterization of two Campylobacter jejuni adhesins for cellular and mucous substrates. Infect. Immun. 53:141-148.

McSweeney L.A. and Dreyfus L.A. (2004). Nuclear localization of the Escherichia coli cytolethal distending toxin CdtB subunit. Cell. Microbiol. 6:447-58. 
Misawa N. and Blaser M.J. (2000). Detection and characterization of autoagglutination activity by Campylobacter jejuni. Infect Immun 68:6168-6175.

Mooney A., Clyne M., Curran T., Doherty D., Kilmartin B. and Bourke B. (2001). Campylobacter upsaliensis excerts a cytolethal distending toxin effect on HeLa cells and T lymphocytes. Microbiol. 147:735-743.

Morooka T., Umeda A. and Amako K. (1985). Motility as an intestinal colonization factor for Campylobacter jejuni. J. Gen. Microbiol. 131:1973-1980.

Murphy C., Carroll C. and Jordan K.N. (2006). Environmental survival mechanisms of the foodborne pathogen Campylobacter jejuni. $J$ Appl Microbiol. 100:623-632.

Myers J.D. and Kelly D.J. (2005). A sulphite respiratory system in the chemoheterotrophic human pathogen Campylobacter jejuni. Microbiology 151:233-242.

Newell D.G., McBride H., Saunders F., Dehele Y. and Pearson A.D. (1985). The virulence of clinical and environmental isolates of Campylobacter jejuni. J. Hyg. 94:45-54.

Nachamkin I., Yang X.H. and Stern N.J. (1993). Role of Campylobacter jejuni flagella as colonization factors for three-day-old chicks: analysis with flagellar mutants. Appl. Environ. Microbiol. 59:1269-1273.

Novik V., Hofreuter D. and Gala'n J.E. (2010) Identification of Campylobacter jejuni Genes Involved in Its Interaction with Epithelial Cells Infection and Immunity. 78:3540-53. 
Ormonde P., Horstedt P., Toole.R.O. and Milton D.L. (2000). Role of motility in adherence to and invasion of a fish cell line by Vibrio anguillarum. J. Bacteriol. 182:2326-2328.

Parkhill J., Wren B.W., Mungall K., Ketley J.M., Churcher C., Basham D., Chillingworth T., Davies R.M., Feltwell T., Holroyd S., Jagels K., Karlyshev A.V., Moule S., Pallen M.J., Penn C.W., Quail M.A., Rajandream M.A., Rutherford K.M., van Vliet A.H., Whitehead S and Barrell BG (2000). The genome sequence of the food-borne pathogen Campylobacter jejuni reveals hypervariable sequences. Nature. 403:665-668

Pavlovskis O.R., Rollins D.M., Haberberger R.L.Jr., Green A.E., Habash L., Stroko S. and Walker R.I. (1991). Significance of flagella in colonization resistance of rabbits immunized with Campylobacter jejuni. Infect. Immun. 59:2259-2264.

Pei Z.M. and Blaser J. (1993). PEB1, the major cell-binding factor of Campylobacter jejuni is a blood homolog of the binding component in gramnegative nutrient transport systems. J. Biol. Chem. 268:18717-18725.

Pickett C.L. and Whitehouse C.A. (1999). The cytolethal distending toxin family Trends Microbiol. 7:292

Purdy D., Buswell C.M. and Hodgson A.E. (2000). Characterisation of cytolethal distending toxin (CDT) mutants of Campylobacter jejuni J. Med. Microbiol. 49:473479.

Rechkemmer G., Ronnan K. and Engelhardt W. (1988). Fermentation of polysaccharides and absorption of short-chain fatty acids in the mammalian hindgut. Comp Biochem Physiol 90:563-568. 
Rees J.H., Thompson R.D., Smeeton N.C. and Hughes R.A.C. (1998). An epidemiological study of Guillain-Barré syndrome in South East England. J Neurol Neurosurg Psychiatry. 64:74-77.

Reid A.N., Pandey R., Palyada K., Naikare H. and Stintzi A. (2008). Identification of Campylobacter jejuni genes involved in the response to acidic $\mathrm{pH}$ and stomach transit. Appl Environ Microbiol. 74:1583-1597.

Rivera-Amill V., Kim B. J., Seshu J. and Konkel M. E. (2001). Secretion of the virulence-associated Campylobacter invasion antigens from Campylobacter jejuni requires a stimulatory signal. $J$ Infect Dis 183:1607-1616.

Rosario M.M., Fredrick K.L., Ordal G.W. and Helmann J.D. (1994). Chemotaxis in Bacillus subtilis requires either of two functionally redundant CheW homologs. $J$ Bacteriol 176:2736-2739.

Rozee K.R., Cooper D., Lam K. and Costerton J.W. (1982). Microbial flora of the mouse ileum mucous layer and epithelial surface. Appl. Environ. Microbiol. 43:1451-1463.

Russell R.G., O’Donnoghue M., Blake D.C. Jr., Zulty J. and DeTolla L.J. (1993). Early colonic damage and invasion of Campylobacter jejuni in experimentally challenged infant Macaca mulatta. J. infect. Dis.168:210-5

Russell R. G and Blake D. C. Jr (1994). Cell association and invasion of Caco-2 cells by Campylobacter jejuni. Infect Immun 62:3773-3779.

Schmidt-Ott R., Pohl S., Burghard S., Weig M. and Groß U. (2005). Identification and characterization of a major subgroup of conjugative Campylobacter jejuni plasmids. J Infect 50:12-21 
Sellars M.J., Hall S.J. and Kelly D.J. (2002). Growth of Campylobacter jejuni supported by respiration of fumarate, nitrte, nitrite, trimethylamine-N-oxide or dimethylsulfoxide requires oxygen. J Bacteriol 184:4187-4196.

\section{Shenker B.J., McKay T., Datar S., Miller M, Chowhan R. and Demuth D.} (1999). Actinobacillus actinomycetemcomitans immunosuppressive protein is a member of the family of cytolethal distending toxins capable of causing a G2 arrest in human T cells. J. Immunol. 162:4773-80.

Skirrow M.B. and Blaser M.J. (1995). Campylobacter jejuni. In: Blaser M.J., Smith P.D., Ravdin J.I., Greenberg H.B., Guerrant R.L., eds. Infections of the gastrointestinal tract. New York City: Raven Press. 825-48.

Smith J.L. and Bayles D.O. (2006). The contribution of cytolethal distending toxin to bacterial pathogenesis. Crit Rev Microbiol. 32:227-248.

Sommerlad S.M. and Hendrixson D.R. (2007). Analysis of the roles of FlgP and FlgQ in flagellar motility of Campylobacter jejuni. J. Bacteriol. 189:179-186.

Sorvillo F.J., Lieb L.E. and Waterman S.H. (1991). Incidence of campylobacteriosis among patients with AIDS in Los Angeles County, J. Acquir. Immune Defic. Syndr. 4:598-602.

Spradling A.C. and Rubin G.M. (1982). Transposition of cloned P elements into Drosophila germ line chromosomes. Science. 218:341-347

Schneeberger E.E. and Lynch R.D. (2004). The tight junction: a multifunctional complex. Am. J. Physiol. Cell Physiol. 286:1213-1228. 
Stintzi A. (2003). Gene expression profile of Campylobacter jejuni in response to growth temperature variation. J Bacteriol. 185:2009-2016.

Szymanski C.M., King M., Haardt M. and Armstrong G.D. (1995). Campylobacter jejuni motility and invasion of Caco-2 cells. Infect. Immun. 63:4295-4300.

Takata T., Fujimoto S. and Amako K. (1992). Isolation of nonchemotactic mutants of Campylobacter jejuni and their colonization of the mouse intestinal tract. Infect Immun 60:3596-3600.

Tauxe R.V., Nachamkin I., Blaser M.J. and Tompkins L.S. (1992). Epidemiology of Campylobacter jejuni infections in the United States and other industrialized nations. American Society for Microbiology. 121-153.

Thies F.L., Karch H., Hartung H.P. and Giegerich G. (1999). Cloning, sequencing and molecular analysis of the Campylobacter jejuni groESL bicistronic operon. Microbiol 145:89-98.

Vegge C.S., Brondsted L., Li Y.P., Bang D.D. and Ingmer H. (2009). Energy taxis drives Campylobacter jejuni towards the most favourable conditions for growth. Appl Environ Microbiol. 75:5308-5314.

Van D.K., Pasmans F., Van I.F., Ducatelle R. and Haesebrouck F. (2008). Butyrate protects Caco-2 cells from Campylobacter jejuni invasion and translocation. British Journal of Nutrition. 100:480-484.

Wassenaar T.M., Bleumink-Pluym N.M.C. and van der Zeijst, B.A.M. (1991). Inactivation of Campylobacter jejuni flagellin genes by homologous recombination demonstrate that flaA but not flaB is required for invasion. EMBO J.10:20552061. 
Wassenaar T.M. and Blaser M.J. (1999). Pathophysiology of Campylobacter jejuni infections of humans. Microbes Infect. 1:1023-33.

Weerakoon D.R., Borden N.J., Goodson C.M., Grimes J. and Olson, J.W. (2009). The role of respiratory donor enzymes in Campylobacter jejuni host colonization and physiology. Microb Pathog. 47:8-15.

Whyte K M., Cowley D, Madden R.H, Moran L, Scates P, Carroll C., Leary A.O., Fanning.S, Collins J.D, McNamara E, Moore J.E. and Cormican M. (2004). occurrence of campylobacter in retail foods in ireland. Inter. J. of food microbiology .1:111-8.

Williams S.B. and Stewart V. (1999). Functional similarities among two component sensors and methyl-accepting chemotaxis proteins suggest a role for linker region amphipathic helices in transmembrane signal transduction. $\mathrm{Mol}$ Microbiol. 33:1093-1102.

Whitehouse C.A., Balbo P.B., Pesci E.C., Cottle D.L., Mirabito P.M. and Pickett C.L (1998). Campylobacter jejuni cytolethal distending toxin causes a $\mathrm{G}_{2}$ phase cell cycle block Infect Immun. 66:1934-40

Woodall C.A., Jones M.A., Barrow P.A., Hinds J., Marsden G.L., Kelly D.J., Dorrell N., Wren B.W. and Maskell D.J. (2005). Campylobacter jejuni gene expression in the chick cecum: evidence for adaptation to a low-oxygen environment. Infect Immun. 73:5278-5285.

Yamazaki W., Taguchi M., Kawai T., Kawatsu K., Sakata J., Inoue K. and Misawa N. (2009). Comparison of Campylobacter jejuni and Campylobacter coli detection in naturally contaminated chicken meat samples between loop-mediated isothermal amplification assay and conventional culture methods. Appl Environ Microbiol. 75:1597-603. 
Yao R., Burr D.H., Doig P.,Trust T.J., Niu H. and Guerry P. (1994). Isolation of motile and non-motile insertional mutants of Campylobacter jejuni: the role of motility in adherence and invasion of eukaryotic cells. Mol. Microbiol. 14:883-893.

Yao R., Burr D.H. and Guerry P. (1997). CheY-mediated modulation of Campylobacter jejuni virulence. Mol. Microbiol. 23:1021-1031.

Young V.B., Knox K.A., Pratt J.S., Cortez J.S., Mansfield L.S., Rogers A.B., Fox J.G. and Schauer, D.B. (2004). In vitro and in vivo characterization of Helicobacter hepaticus cytolethal distending toxin mutants. Infect Immun 72:2521-2527.

Young K.T., Davis L.M. and Dirta V.J. (2007). Campylobacter jejuni: molecular biology and pathogenesis. Nat. Rev. Microbiol. 5:665-79

Yrios J.W. and Balish E. (1986). Colonization and infection of athymic and euthymic germfree mice by Campylobacter jejuni and Campylobacter fetus subsp. fetus. Infect. Immun. 53:378-383. 


\section{CURRICULUM VITAE}

Name:

Abdul Malik

DOB:

01-02-1976

Place of Birth

Quetta, Pakistan

Email Address: abdulmaliktareen@yahoo.com

\section{ACADEMIC QUALIFICATIONS}

Ph.D (cont.) Institute for Medical Microbiology, Center for Hygiene and Human Genetics School of Medicine, University of Göttingen, Germany

MS Biotechnology \& Informatics BUITMS, Pakistan (2006)

DVM University of Agriculture, Faisalabad, Pakistan (2000)

Certificate National Training Workshop on sample collection, storage and transportation for the diagnosis of transboundary animal disease (RP, FMD and PPR) Islamabad, Organized by FAO United Nation.

Water Harvesting and Water shed Management by IUCN International Pakistan.

DIT Diploma in Information Technology,

\section{KNOWLEDGE OF LANGUAGES}

English, German, Urdu, Pashto,

\section{PROFESSIONAL EXPERIENCE}

PhD Student (09/2007-to date)

Lecturer, CASVAB, University of Balochistan, Pakistan (08/2005)

Responsibilities include:

1 Teaching to the M.Phil student.

2 Research work.

\section{MEMBERSHIP IN PROFESSIONAL SOCIETIES}

Der Deutschen Gesellschaft für Hygiene und Mikrobiologie

Society of Microbiology, Pakistan.

Member board of studies of Physiology, Pakistan. 


\section{TRAINING AND SEMINAR}

Medical Biodefence conference 2009, Munich, Germany.

61. Jahrestagung der deutschen Gesellschaft für Hygiene und Mikrobiologie 2009 Göttingen Germany.

60. Jahrestagung der DGHM 2008, Dresden, Germany.

Receive Training on the Participatory Disease Surveillance Project funded by European Union, Food and Agriculture Organization of United Nation in collaboration with Ministry of Agriculture and Livestock Government of Pakistan at Tando Mohammad Khan Sindh Pakistan. 